\title{
Effect of housing in an enriched environment on the recovery from experimental inflammatory pain
}

Citation for published version (APA):

Gabriel, A. F. (2009). Effect of housing in an enriched environment on the recovery from experimental inflammatory pain. [Doctoral Thesis, Maastricht University]. [Maastricht University]. https://doi.org/10.26481/dis.20091022ag

Document status and date:

Published: 01/01/2009

DOI:

10.26481/dis.20091022ag

Document Version:

Publisher's PDF, also known as Version of record

\section{Please check the document version of this publication:}

- A submitted manuscript is the version of the article upon submission and before peer-review. There can be important differences between the submitted version and the official published version of record. People interested in the research are advised to contact the author for the final version of the publication, or visit the DOI to the publisher's website.

- The final author version and the galley proof are versions of the publication after peer review.

- The final published version features the final layout of the paper including the volume, issue and page numbers.

Link to publication

\footnotetext{
General rights rights.

- You may freely distribute the URL identifying the publication in the public portal. please follow below link for the End User Agreement:

www.umlib.nl/taverne-license

Take down policy

If you believe that this document breaches copyright please contact us at:

repository@maastrichtuniversity.nl

providing details and we will investigate your claim.
}

Copyright and moral rights for the publications made accessible in the public portal are retained by the authors and/or other copyright owners and it is a condition of accessing publications that users recognise and abide by the legal requirements associated with these

- Users may download and print one copy of any publication from the public portal for the purpose of private study or research.

- You may not further distribute the material or use it for any profit-making activity or commercial gain

If the publication is distributed under the terms of Article $25 \mathrm{fa}$ of the Dutch Copyright Act, indicated by the "Taverne" license above, 


\begin{abstract}
Effect of housing in an enriched environment on the recovery from experimental inflammatory pain
\end{abstract}


(CAnne F.M.I. Gabriel, Maastricht 2009

Typesetting by Michał Sławiński

ISBN 978-90-9024597-3

Printed in Poland 


\title{
Effect of housing in an enriched environment on the recovery from experimental inflammatory pain
}

\author{
Proefschrift
}

ter verkrijging van de graad van doctor aan de Universiteit Maastricht, op gezag van Rector Magnificus, Prof. Mr. G.P.M.F. Mols volgens het besluit van het College van Decanen, in het openbaar te verdedigen op

donderdag 22 Oktober 2009 om 16:00 uur

door

\section{Anne F.M.I. GABRIEL}




\section{Promoter}

Pr dr M. Marcus

\section{Co-promoter}

Dr E. Joosten

\section{Assessment Committee}

Pr dr J. Vles, Chairman

Pr dr L. Arendt-Nielsen, Aalborg, Denmark

$\operatorname{Pr}$ dr A. Dahan, Leids UMC

Pr dr M. Peters

Pr dr J. van Overbeeke 
The refreshing pleasure from the first view of nature, after the pain of illness, and the confinement of a sick-chamber, is above the conceptions, as well as the descriptions, of those in health. Ann Radcliffe, The Mysteries of Udolpho, 1764 



\section{Table of content}

Chapter 1

Introduction

Chapter 2 The CatWalk method: a detailed analysis of behavioral

changes after acute inflammatory pain in the rat

Chapter 3 The CatWalk method: assessment of mechanical allodynia in experimental chronic pain?

Chapter 4 The CatWalk method: a sensitive and objective tool

for preclinical assessment of analgesic drugs

Chapter 5

Environmental housing affects the duration of

75

mechanical allodynia and the spinal astroglial activation

in a rat model of chronic inflammatory pain

Chapter 6 Preoperative housing in an enriched environment significantly reduces the duration of post-operative pain in a rat model of knee inflammation

Chapter 7 Enriched environment and the recovery from inflammatory pain: social versus physical aspects and their interaction

Chapter 8

Discussion

Summary

Aknowledgment

Curriculum vitae 


\section{Abbreviations}

$\begin{aligned} \text { 5-HT } & \text { Serotonin } \\ \text { BDNF } & \text { Brain-derived neurotrophic factor } \\ \text { BOS } & \text { Base-of-support } \\ \text { CAR } & \text { Carrageenan } \\ \text { CGRP } & \text { Calcitonin gene-related peptide } \\ \text { COX-2 } & \text { Cyclooxygenase 2 } \\ \text { DPO } & \text { Day post-operation } \\ \text { DRG } & \text { Dorsal root ganglia } \\ \text { EAA } & \text { Essential amino acid } \\ \text { EE } & \text { Enriched environment } \\ \text { FEN } & \text { Fentanyl } \\ \text { GC } & \text { Glucocorticoid } \\ \text { GFAP } & \text { Glial fibrillary acidic protein } \\ \text { HPA axis } & \text { Hypothalamic-pituitary-adrenal axis } \\ \text { IM } & \text { Intra-muscular } \\ \text { NA } & \text { Noradrenalin } \\ \text { NK-1 } & \text { Neurokinin-1 } \\ \text { NMDA } & \text { N-methyl D-aspartic acid } \\ \text { NSAID } & \text { Non steroid anti-inflammatory drug } \\ \text { PAG } & \text { Periaqueducal Grey matter } \\ \text { PCA } & \text { Patient-controlled analgesia } \\ \text { PCEA } & \text { Patient-controlled epidural analgesia } \\ \text { PE } & \text { Physically enriched environment } \\ \text { PG } & \text { Prostaglandin } \\ \text { RVM } & \text { Rostral ventral medulla } \\ \text { SE } & \text { Socially enriched environment } \\ \text { SNS } & \text { Sympathetic nervous system } \\ & \\ \text { PE } & \\ \text { PE } & \end{aligned}$




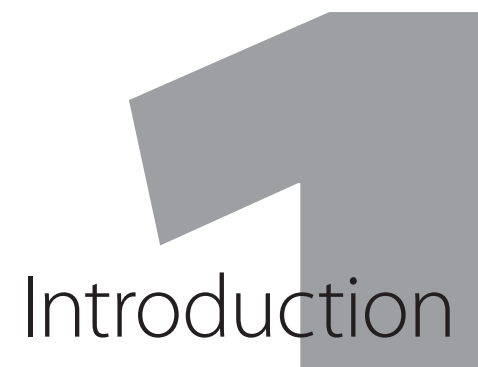




\section{Clinical Inflammatory Pain}

\section{a. Persistent postsurgical pain}

Persistent postsurgical pain is a major clinical problem with significant human and socioeconomic consequences. The last decade has seen an increased attention to pain complaints after several surgical operations. Many reviews report pain from amputation [1], breast surgery [2], inguinal herniotomy [3], thoracotomy [4], cardiac surgergy (sternotomy) [5], cesarean section [6], hysterectomy [7], major joint surgery [8], and other orthopedic procedures [9]. These common procedures lead to persistent pain complaints in $10-50 \%$ of patients, with moderate to severe pain in about $2-10 \%[10]$.

The pathogenic mechanisms are multiple but there are three main factors: preoperative risk factors, intraoperative nerve damage and postoperative inflammation [10]. Hence, postoperative pain can either be due to nerve damage (i.e. neuropathic pain) or to inflammation insult (i.e. inflammatory pain). Although lots of research is done on nerve damage and the possibilities for avoiding or reducing it, the present knowledge on the relative role of inflammation from the surgical area for developing a persistent pain state is very limited. Surgery induces a profound inflammatory response [11]. Indeed, surgical injury is known to stimulate and favor the release of proinflammatory cytokines. In the normal resolution phase of inflammation, neutrophil apoptosis and the subsequent ingestion by macrophages play an important role in limiting the autotoxic potential of this cell [12]. After a surgical intervention, neutrophils can persist at the site of inflammation, cause uncontrolled release of toxic metabolites, or may undergo secondary necrosis, perpetuating and exacerbating the postoperative inflammatory response [11].

Pain control after arthroplasty has been a key concern for most orthopedic surgeons [13]. After a total knee joint replacement, $17 \%$ of patients have been reported to develop arthrofibrosis, due to inflammation and an excessive scar tissue response leading to painful restriction of knee motion [13]. Thus for this type of surgery, postoperative pain is mainly caused by tissue inflammation where the acute normal postoperative inflammation and the subsequent transient inflammatory pain can turn into a chronic inflammation which maintains persistent postoperative pain states.

In the following chapters, description, clinical management and experimental models of inflammatory postoperative pain will be detailed.

\section{b. Post-surgical knee inflammation: the process}

At first, the process will be described, step by step, from the peripheral inflammation insult to the persistent pain state. 


\section{The inflammatory response}

In a surgical wound, inflammation is induced as a result of direct trauma with release of inflammatory mediators (e.g. calcitonin gene-related peptide, Substance P) from tissues and cells (e.g. mast cells, macrophages and lymphocytes) [14]. These inflammatory mediators are able to activate nociceptors (i.e. neurons involved in the pain signaling). Nociceptors, also called primary afferents, are either lightly myelinated A $\delta$ fibers or unmyelinated $\mathrm{C}$ fibers. A $\delta$ fibers are associated with cold and pressure. $\mathrm{C}$ fibers are polimodal and can respond to thermal, mechanical and chemical stimuli. Both are located in peripheral tissues and end up in the dorsal horn of the spinal cord. Antidromic impulses in primary afferents release chemical mediators that further enhance vasodilatation at the site of inflammation. Thus the edema together with the increased capillary permeability enhance the process with further cellular accumulation [14]. Figure 1 gives an overview of the complex neurobiological event associated with inflammation and these will be addressed in more details in the following sections.

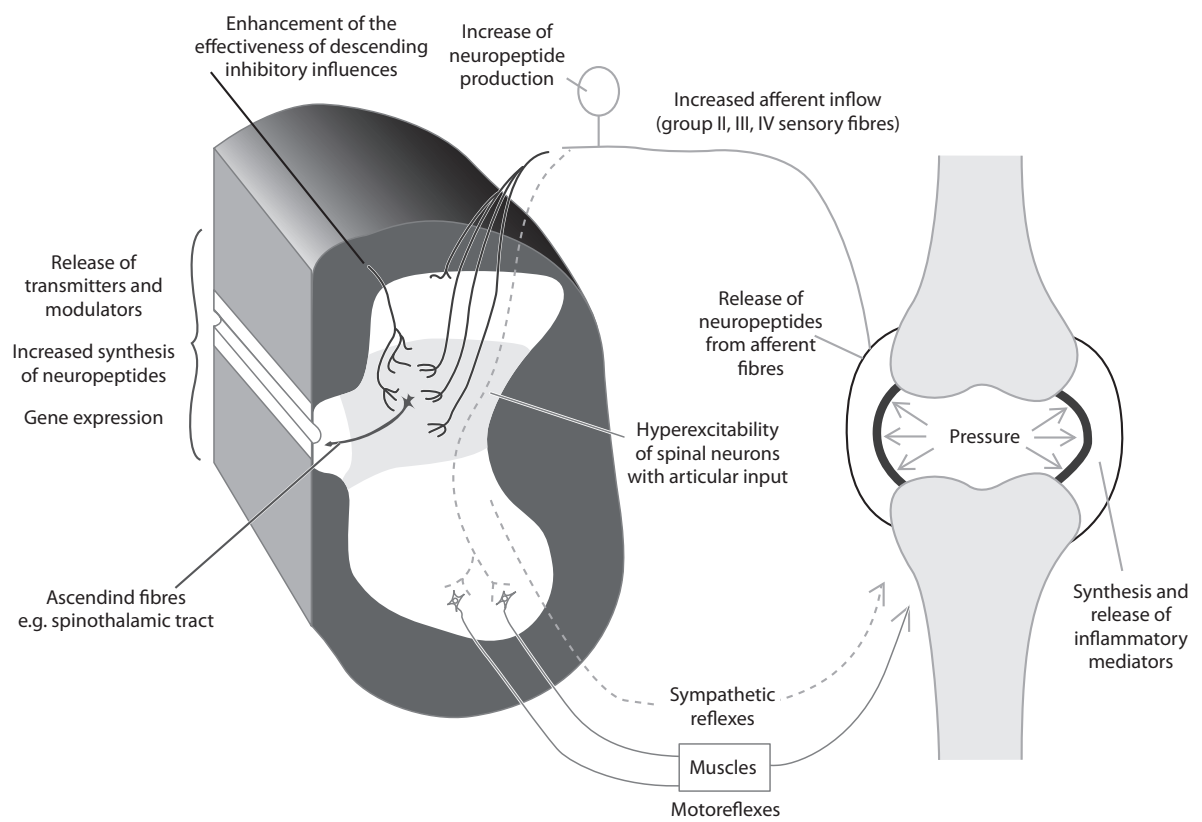

Figure 1: Main neuronal events in the course of chronic knee inflammation. Starting from peripheral release of inflammatory mediators, afferents are activated. They end up in the superficial laminae of the spinal dorsal horn where central sensitization and pain modulation take place [15]. Reproduced with permission from the publisher. 


\section{Peripheral sensitization}

The inflammatory response in the knee leads to accumulation of a large amount of molecules such as peptides and intermediaries from the pathways of arachidonic acid metabolism. These molecules affect the excitability of sensory nerve fibers [16]. It results in a local higher sensitivity: low intensity stimulation normally perceived as nonpainful becomes painful (i.e. allodynia). Similarly, barely painful events become highly painful (i.e. hyperalgesia).

\section{Central sensitization in the dorsal horn}

The dorsal horn is commonly divided in laminae (see schematic representation in Figure 2). Laminae from the spinal cord are organized by the type of information they receive from the body. Laminae I and II, located in the superficial part of the dorsal horn, receive information from peripheral afferent nociceptive fibers. Laminae III and IV receive information about non-noxious stimuli and proprioceptive information. Lamina $\mathrm{V}$ plays an important role in the transmission of noxious information to supraspinal centers [17].

Nociceptive-specific second-order neurons are situated within the dorsal horn of the spinal cord in the superficial laminae on which primary nociceptive afferents synapse. At deeper layers "wide dynamic range neurons" are situated, responding to both nociceptive and non-nociceptive afferent input; these may become sensitized and respond strongly to tactile stimuli [18]. Figure 2 represents a schematic transverse section of the spinal cord including the spinal dorsal horn and nociceptive pathways.

Among others, central sensitization depends on N-methyl D-aspartate (NMDA) receptor activation [19], which exhibit three features: (1) an increased receptor field size; (2) an increased size and duration of neuronal response to a standard stimulus; (3) a reduced threshold, i.e. nociceptive neurons responding to normally subthreshold stimuli. NMDA receptors seem to be of minor importance during normal transient processing of nociceptive signals. A prolonged nociceptive stimulation, i.e. a prolonged release of glutamate or neurokinin 1 (NK-1), results in activation of NMDA receptors (see Figure 3). Thus, the NMDA receptors are important in the persistence of acute clinical pain, and also chronic pain, together with the encoding of pain in memory [19].

\section{Cellular events at the spinal level}

An important factor, besides the important neuronal implication, is the well-documented contribution of immune-like glial cells to pain processing. Astrocytes and microglia are described to play a role in pain facilitation: they are able to modulate neuronal synaptic function and neuronal excitability through several mechanisms [20,21] (Figure 3). 


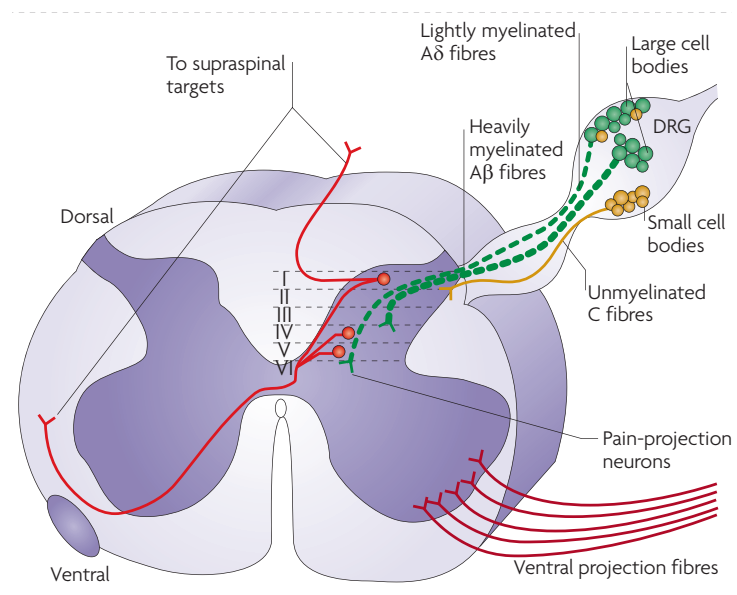

Figure 2: Normal pain signaling in the body is transmitted to the spinal cord dorsal horn through nociceptors. Nociceptive pain, such as a pin prick to the foot, leads to the release of pain transmitters from primary afferent terminals that project onto pain-projection neurons primarily to laminae I, IV and $\mathrm{V}$ in the spinal cord dorsal horns. It is notable that $A \beta, A \delta$ and $C$ fibers additionally project to laminae $I I-V I$ to a much lesser extent, but can alter pain-projection neuron activity. However, when tissue injury and skin inflammation ensue, chronic inflammatory signals are released from surrounding cells at the peripheral nerve terminal and lead to the sensitization of the nociceptors. Such factors include, but are not limited to, adenosine and its related mono- or polyphosphorylated compounds (AMP, ADP and ATP), bradykinin, glutamate, histamine, substance P, CGRP and tumor-necrosis factor-a. Nociceptive signaling from the dorsal root ganglia (DRG) is then relayed to the dorsal spinal cord, brain stem and brain, where the experience of pain occurs [17]. Reproduced with permission from the publisher.

In their normal basal state, microglia are quiescent and astrocytes predominantly fulfill "housekeeping" functions such as extracellular ion regulation. Under these conditions, microglia and astrocytes have little-to-no influence on pain sensitivity [22, 23]. Various drugs that suppress glial function have failed to alter responsitivity to heat or mechanical stimuli in normal animals [22, 24, 25]. However, upon activation, microglia and astrocytes are able to release neuroactive substances that may enhance the excitability of pain transmission neurons [26,27]. Spinal microglia are the earliest glial cell type activated in response to peripheral inflammation and injury $[25,28]$. The initial microglial activation then recruits and activates astrocytes [29]. Thus, glia do not just induce pain facilitation but are key mediators in pain maintenance as well [30]. Activation of glial cells is accompanied by major cellular changes that allow its detection.

Among others, activated microglial cells present a higher expression of the complement receptor $\mathrm{C} 3 \mathrm{~b}$ (recognized by the monoclonal antibody OX-42) [32] whereas astrocytes activation is described to be correlated with an increase in the Glial Fibrillary Acidic Protein (GFAP) [33]. 
a

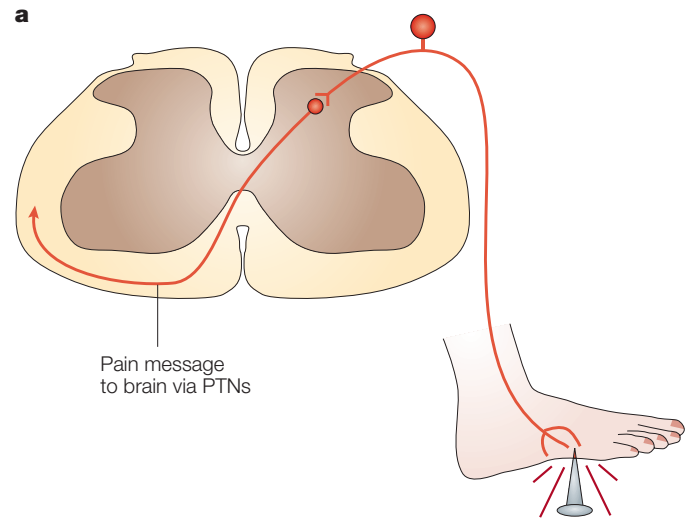

Pain stimulus b

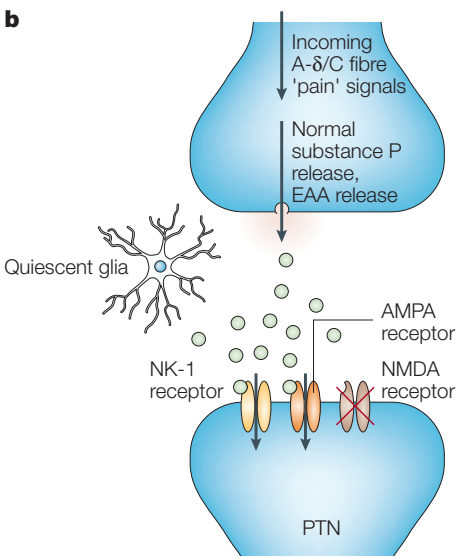

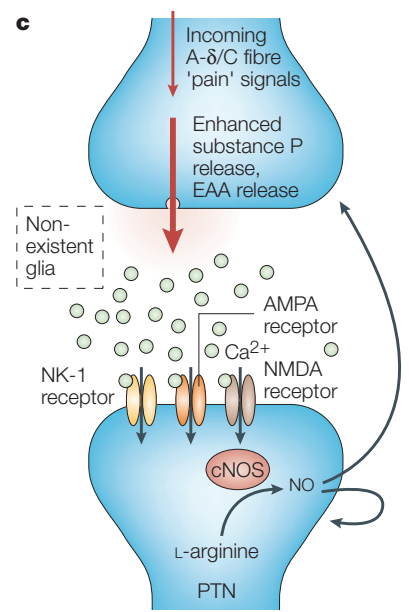

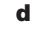

Viruses and bacteria

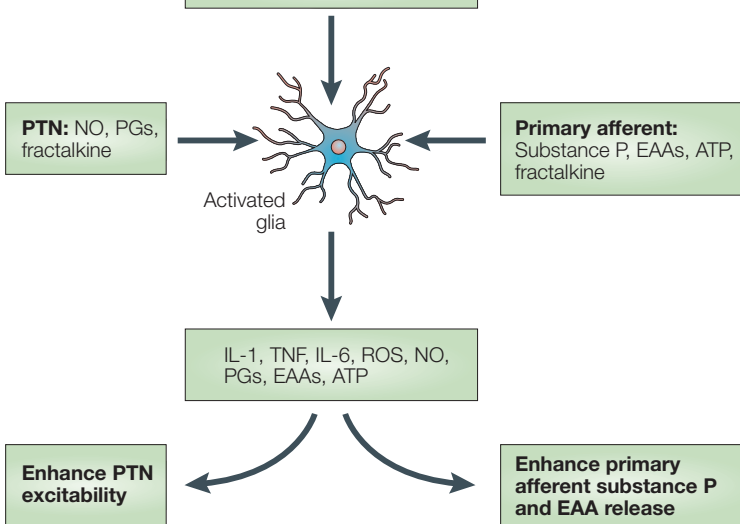

Figure 3: Classical and non-classical views of pain transmission and pain modulation (a). Classical pain transmission pathway when a noxious (painful) stimulus is encountered, such as stepping on a nail as shown. (b): Normal pain. Under basal conditions, pain is not modulated by glia. Glia are quiescent, and thus not releasing pain modulatory levels of neuroexcitatory substances. (c) Pain facilitation: classical view. In response to intense and/or prolonged barrages of incoming "pain" signals, the post-transmission neurons (PTNs) become sensitized and overrespond to subsequent incoming signals. NMDA channels and nitric oxide (NO) are described as major players in creating pain facilitation in this neuronally driven model whereas glia have not been considered to be involved. (d): Pain facilitation: new view. Here, glial activation is conceptualized as a driving force for creating and maintaining pain facilitation. The role of glia is superimposed on the NMDA-NO-driven neuronal changes. Glia are activated by three sources: bacteria and viruses; substance P, EAAs, fractalkine, and ATP released by A-delta and/or C fiber presynaptic terminals (shown here) or by brain-to-spinal cord pain enhancement pathways (not shown); and NO, prostaglandins (PGs) and fractalkine released from PTNs. Following activation, microglia and astrocytes cause PTN hyperexcitability and the exaggerated release of substance P and EAAs from presynaptic terminals [31]. Reproduced with permission from the publisher. 


\section{Central pain modulation}

The transmission of information from primary afferents to secondary neurons in the spinal dorsal horn is subject to "gating" (neuromodulation). Nociceptive traffic is affected both directly at the spinal cord level as well as a result of descending modulation.

Local modulation results from release of neurotransmitters by interneurons or axons synapsing with second-order neurons. The most important receptors for neurotransmitters in nociceptive afferents are the following: opioid receptors [34]; alpha-adrenoreceptors [35]; GABA and glycine receptors [36].

Further nociceptive transmission between the spinal cord and the brain (e.g. spinothalamic or post-synaptic dorsal column pathways) have already been described [37]. Descending pathways, from brain to spine, have also been investigated. Brainstem nuclei included in the descending circuitry include the periaqueductal grey region (PAG) that projects both to the rostral ventral medulla (RVM), a primary source of serotonergic input to the spinal cord, and the dorsolateral pontine tegmentum, a primary source of noradrenergic input to the spinal cord [38]. However, description of the role of subcortical structures, and the way in which the cortex appreciates pain, still needs further investigations [39].

\section{Persistent pain states}

In normal cases, postsurgical pain declines rapidly over the first few postoperative days, depending on the type of surgery. This type of pain state is referred to as acute. Nevertheless, pain may persist in some patients, which is often related to the surgery itself, due to infection or hematoma, i.e. due to inflammation. It might also occur in neuropathic pain state. In both the inflammatory and neuropathic pain states, it is referred to as persistent pain. First indications on prevalence and predictors of postoperative persistent pain have already been described [40]. However, prolonged pain syndrome after surgery is not well understood and still needs to be extensively studied by either surgeons or anesthesiologists. After 3 to 6 months, clinicians refer to such a state as chronic pain state. 


\section{Postsurgical pain management}

\section{a. Treatments of postoperative pain}

Postoperative pain can be managed using a variety of techniques, including intravenous patient-controlled analgesia (IV PCA), intermittent and continuous epidural analgesia, patient-controlled epidural analgesia (PCEA), regional nerve blockade, IV bolus doses of analgesics, intramuscular (IM) injection of analgesics, and oral analgesia [41].

Opioids are the most commonly used analgesics for treatment of moderate-tosevere postoperative pain $[42,43]$ and are widely used in patient-controlled analgesia systems [41, 44]. However, the use of opioids can induce severe side-effects such as breathing depression and the development of tolerance and dependence [45]. Therefore, for the management of less severe postoperative pain, drugs such as paracetamol or non steroid anti-inflammatory drugs (NSAID's) are preferred.

Besides, pre-emptive administration of analgesia can also be used (i.e. administration of the drug before the perception of pain). However, there is conflicting evidence for the efficacy of such a treatment [46,47] since some show it to be ineffective [48-50].

Despite these new techniques, multimodal treatments, and the establishment of new clinical practice guidelines throughout the last decade, effective postoperative pain management remains a significant clinical issue [51].

\section{b. Impact of the environment}

Psychological factors, such as anxiety, have been described to be good predictors of postoperative pain [52]. In that sense, increased attention has been paid to the impact of the environment on the recovery of clinical patients. In 1984, it was described that patients assigned in hospital room with a window view on nature had shorter hospital stay, took fewer analgesics and reported less negative comments in nurses' notes than those assigned in a room with window facing a brick building wall [53]. In line with these findings, the concept of 'healing environment' emerged, where not only the professionals would be the healing factor but the experience of the building itself would also have a positive influence on patients $[54,55]$.

The notion of healing space actually dates back to ancient Greece [55]. Temples such as the sanctuary at Epidaurus, the most celebrated healing center of the Classical world, were built for the god Asclepius, the healer god of antiquity. Ill people went to Epidaurus in the hope of having dreams where Asclepius would reveal the cures for ailments. The temple was designed to surround patients with nature, music and art, to restore harmony and restore healing. Among others, many mineral springs in the vicinity may have been used in healing. Sport races and theatric 
competitions were organized around the temple and the place was mainly famous for its poetry contests [56]. Later, in 1860, Florence Nightingale, a pioneering nurse, claimed ventilation and fresh air as "the very first canon of nursing", along with elimination of unnecessary noise, proper lighting, warmth, and clean water [57]. In the last several decades, the growth of technological advances needs for infection control, efficiency, and patient safety have caused the architecture and design of these buildings to become austere, noisy, antiseptic smelling and unattractivelooking facilities. However, as the need for hospitals to become more competitive arose, aesthetically pleasing design has provided not only a competitive edge but has become a solid contributor to improving patient and staff satisfaction.

Today, the generally accepted components of a healing space are an architecture that provides access to nature, light, good air quality, and privacy; pleasant or positive distractions; and reduction of environmental stressors such as unnecessary noise, and toxic or harmful substances. In addition to these components, aroma, music, color, and artwork are also often used to supplement the optimal healing space [55].

In the last decades, clinical studies have demonstrated significant effects of different components from the patients environment on the intensity and/or duration of pain. Indeed, as previously mentioned, the concept of 'healing environment' reemerged from the past, where not only the professionals are the healing factor but also the experience of the building itself $[54,55]$. Just as in ancient Greece, such an environment includes both physical and social enrichment. First, regarding the physical approach, stimulation of the patient's five senses has been shown to significantly decrease the intensity and/or duration of pain, e.g. reducing noise [58] or using musical therapies [59]. Besides, patients facing with pleasant nature images have less anxiety and require fewer strong pain medication doses as compared to patients with abstract art images or no pictures at all [60]. Colors are also described to affect patients mood and wellness and ocular light therapy in which light is projected through colored filters into the eyes is used to treat headaches and anxiety [60]. On the other hand, from a more social point of view, health benefits of positive distraction (i.e. humor and entertainment) have been described, among which is a significant decrease in the use of pain-relieve medication after surgery [61].

\section{Experimental Inflammatory Pain}

\section{a. Animal models of inflammatory pain}

Models of experimental postoperative inflammation are necessary in order to study the neurobiology of knee inflammation as well as the development of new treatments. In dogs, cats, rabbits, rats and pigeons, acute forms of inflammation 
in the joint can be induced by injection of crystals such as urate or kaolin and/ or through the injection of carrageenan into the cavity of the joint [62-67]. These injections induce the synthesis and release of inflammatory mediators which lead to an edema and a rapid infiltration of polymorphonuclear granulocytes within the first hours [64]. Behavioral signs of hyperalgesia appear within 1 to 3 hours, such as inactivation of the leg during walking and quick removal of the leg when the inflamed join is pressed, suggesting a state of mechanical hyperalgesia. The acute inflammatory process may change its histopathological features with time and become more chronic [64].

Furthermore, Complete Freund's Adjuvant (CFA) is often used in experimental inflammation research. It has an immunological origin and is commonly used to study the effect of chronic inflammation in joints. At first, a CFA injection into the foot pad, tail base or lymph nodes produces an acute local inflammatory reaction which develops within the first few hours after inoculation. It becomes chronic after 4-5 days and declines after 3 to 5 weeks. During the second week a diffuse inflammatory reaction develops in the distal joints of the limb. Lesions may also develop at other sites in the body, such as eyes, ears or tail $[68,69]$. After 4 weeks, the inflammatory lesions usually declines but may recur spontaneously at later time points.

When Formalin, a solution of formaldehyde in water, is injected into the plantar surface of the rat hindpaw, it produces complex response patterns that last for approximately 1 hour. Two phases of nociceptive behavior are typically described: the first or acute phase lasts for about $5 \mathrm{~min}$ and is followed by a longer-lasting, more persistent phase (about $40 \mathrm{~min}$ ) that is characterized by shaking or licking of the paw. It is generally agreed that the first phase results at least in part from direct activation of primary afferent fibers, both low-threshold mechanoreceptive and nociceptive types [70]. The second phase has been suggested to reflect the combined effect of afferent imput and central sensitization in the dorsal horn [71].

The various inflammatory agents used in studies of experimental postoperative pain present different pathologies and follow different time courses. Hence, selection of the experimental model highly depends on the characteristics of the inflammatory agents used.

\section{b. Carrageenan-induced inflammation model}

In the present investigation, a carrageenan-induced inflammation model is used. This model was chosen because injection of carrageenan into the right knee of an adult rat leads to the development of a non immune and highly reproducible knee inflammation [72]. Moreover, this model can be used to study both acute and chronic inflammation phases, depending on the concentration of carrageenan injected [73]. 
At first, when injected into the knee joint, carrageenan leads to the synthesis and release of inflammatory mediators (i.e. glutamate and nitric oxide metabolites), which cause edema and rapid infiltration of neutrophils within the first few hours [15]. The local changes occurring after carrageenan insult are likely to be responsible for the sensitization of the peripheral nociceptors and primary afferents, which then contribute to the development of secondary hyperalgesia, along with central changes $[74,75]$.

Indeed, injection of carrageenan has been shown to induce cyclooxygenase-2 (COX-2) and produces prostaglandin E2 locally [76, 77], induces COX-2 mRNA in the lumbar spinal cord [78] and releases glutamate, aspartate, substance $\mathrm{P}$, nitric oxide and prostaglandin E2 in the dorsal horn [79-83]. Carrageenan has also been found to cause an acute increase in immunoreactive CGRP and substance P in the spinal cord that lasts through at least 1 week $[84,85]$. Some or all of these spinal changes could be responsible for the central sensitization occurring spinally, following carrageenan-induced inflammation of a joint or muscle [86-88]. Supraspinal involvement in secondary hyperalgesia produced by peripheral tissue injury, including knee joint carrageenan, has been reported $[89,90]$.

It was previously described that most of the carrageenan is removed within $48 \mathrm{~h}$ from the knee joint [64] and possibly even faster from other tissues. Nevertheless, secondary hyperalgesia produced by carrageenan persists even after $48 \mathrm{~h}$ and transforms into long-lasting hyperalgesia (weeks). Histopathological examination of the tissues shows inflammatory changes that parallel the long-lasting hyperalgesia observed [73]. Therefore, the long-lasting hyperalgesia is most probably maintained by the chronic inflammation observed in tissues.

The duration and contralateral spread of long-lasting hyperalgesia depends on the severity of initial inflammation produced by carrageenan in relation to the site of injection [73]. In that study, 1mg CAR was injected into the knee joint and produced short-lasting hyperalgesia that remained ipsilateral whereas $0.3 \mathrm{mg}$ CAR injected into the knee joint had no effect on hyperalgesia. On the other hand, a $3 \mathrm{mg}$ CAR knee injection produced hyperalgesia which lasted 8 weeks and spread to the contralateral side after 1-2 weeks. A similar pattern of inflammatory changes was observed histologically. Following an injection of $3 \mathrm{mg}$ of CAR, acute inflammation was observed for the first $24 \mathrm{~h}$ with edema and neutrophilic infiltration evident as early as $4 \mathrm{~h}$. At 1 week, the inflammation converted to primarily a macrophage response with scattered mast cells. All together, their data indicate that animals injected with either 1 or $3 \mathrm{mg}$ carrageenan in the knee joint can be used as models of acute inflammation through $24 \mathrm{~h}$ and of chronic inflammation after 1 week. Furthermore, $3 \mathrm{mg}$ CAR injected into deep tissues produces hyperalgesia that spreads to the contralateral side, at the same time period as the inflammation transforms from acute to chronic. 
In conclusion, the carrageenan model is described as a model of acute inflammatory pain during the first days which later develops into a model of persistent pain after one to two weeks that lasts up to 3 to 8 weeks, depending on the amount of carrageenan injected [73].

\section{c. Pain measurement in experimental models}

Objective assessment of pain is a major issue in experimental pain studies. Due to the absence of verbal communication in animals, pain analysis needs to be based on either physiological or behavioral analysis, the last one being described as the most specific [91]. Many experimental models for acute and chronic pain have been developed. Within these models, pain is generally assessed using a variety of tests which almost exclusively are based on the response to pain-inducing actions. For assessment of mechanical allodynia the most commonly used technique is the von Frey test [92, 93]. Animals are placed in a plastic cage with a metal mesh floor, allowing them to move freely. Filaments of various thicknesses are applied to the mid-plantar surface of both animal hind paws, until the filament bends. Filaments are applied in ascending order, and the smallest filament that elicits a positive response (i.e. a paw withdrawal) is considered the threshold stimulus. Thermal allodynia is mainly measured by the Hargreaves test. A radiant heat source is focused onto the plantar surface of the animal hindpaw. Paw withdrawal latencies are measured, manually or automatically. Usually, a maximal cutoff of about $25 \mathrm{~s}$ is used to prevent tissue damage [94, 95].

These reflexive measures of nociception are mainly mediated via spinal and spinobulbar pathways [96]. However, perception of pain includes higher level cerebral processing of nociceptive input [39]. Therefore, behavioral tests using higher nervous centers have been developed: the operant tests. Operant tests have been conceived where the animal has to tolerate pain in order to get access to a reward (e.g. food). Both thermal and mechanical sensitivity can be measured. In this type of test, the response measured depends on the net effect of two opposing behavioral driving forces (e.g. pain and hunger). The driving force 'pain' is leading when the response (escape) is observed. The escape latency is measured as a marker of the pain sensitivity. Longer escape latencies from a stimulus indicate reduced pain sensitivity. For instance, in the escape avoidance paradigm, animals must associate the application of the mechanical stimulus to the hyperalgesic paw with the preferred dark area of the test chamber [97]. The advantage of these operant tests of thermal and mechanical sensitivity is that response can be attributed to pain processing throughout the neuraxis, including the cerebrum, rather than the spinal or spinal-brainstem-spinal circuits that mediates reflexive responses [96]. Moreover, due to the difference in the stimulated circuits, dichotomous effects of pain killers in operant versus reflexive tests have been observed in rats. Hence, effects of systemic 
morphine on operant escape responses of rats to thermal stimulation were compared directly with effects on innate licking and guarding responses [98]. Administration of 0.5 to $1.5 \mathrm{mg} / \mathrm{kg}$ morphine attenuated escape from nociceptive heat and in distinct contrast, licking and guarding responses to heat were enhanced by these doses. The authors conclude that although innate responses have been used almost exclusively for testing pain and antinociception because the animals do not have to be trained, their results address new considerations about the techniques to use and the object of their measure.

Finally, in the clinical situation, pain is generally evaluated based on how patients handle pain during normal life [99]. Similarly, various attempts have recently been undertaken to partly analyse gait changes in an experimental model of acute pain in order to analyse how the animal handles pain during normal behavior, like gait. A device was developed to measure the weight load on each leg while the rat is walking [100]. After an injection of $1 \mathrm{mg}$ of carrageenan into the rat knee, they observed a significant reduction of weight load on the affected leg. The minimum level in load was observed at 4 hours after injection. It gradually returned to the preinjection level by the fifth day. Besides, Coulthard and co-workers (2003) measured gait changes as an objective marker of persistent pain using video recordings from spontaneously walking rats [101]. A bilateral rear intraplantar injection of $6 \mathrm{mg}$ of carrageenan resulted in temporal and spatial changes in gait over a 90 minutes period. Changes included a reduction of velocity and stride length as well as an increase

(A)
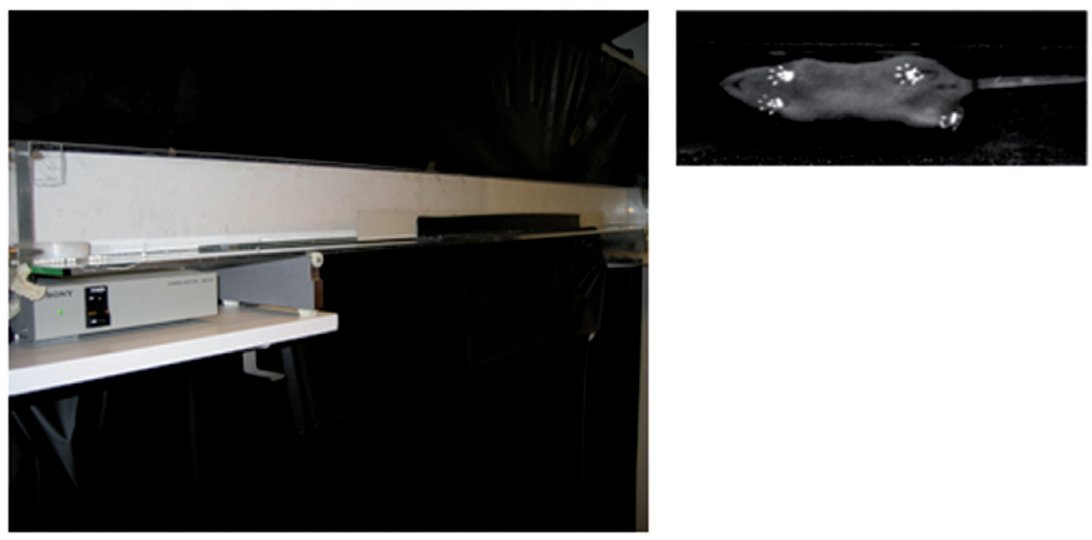

(B)

Figure 4: $(A)$ CatWalk pathway (length $=1 \mathrm{~m}$ ). Rats are trained to cross the pathway with a constant speed. Animals are motivated by the mean of food award, placed at the end of the pathway. (B) View from the camera placed underneath the pathway. Light is shinning through the glass plate and is reflected downward at any place of contact between the animal and the plate. The camera is connected to a computer which records the whole run. Images are analysed by the CatWalk software. 
in dual stance duration. However the changes they observed in velocity have been documented to affect several gait parameters $[102,103]$ and consequently make any comparison of those perilous.

In this respect, the CatWalk technique, a computer-assisted method of locomotor analysis, is interesting because it allows the rapid quantification of individual paw parameters as well as parameters related to interlimb coordination both in view of the crossing time (velocity) [104] (Figure 4). This method has been originally developed a few years ago to assess locomotor deficits in spinal cord injury models [105]. Recently the CatWalk method has been suggested as a method which allows objective assessment of mechanical allodynia in a model of chronic pain [106]. After the induction of a chronic constriction injury (CCI) in the rat sciatic nerve, Vrinten and Hamers demonstrated that changes in three Catwalk-gait parameters (i.e. stance and swing phases duration and intensity of the paw print) highly correlated with the development of mechanical allodynia as measured by the von Frey test. However, here again, rats were not trained and the influence of the velocity on the different gait parameters during a run was not controlled.

In conclusion, although several attempts have been undertaken, the measurement of pain in experimental animals is still a major issue and objective assessment is not being achieved yet.

\section{d. Impact of the environment on pain in experimental models}

The outcomes of clinical environmental interventions is not yet clearly defined and understood and the need of experimental models remains in order to further investigate and optimize the impact of the environment on the recovery from postoperative pain. In experimental research, the main paradigm to study the effect of housing conditions is the enriched environment (EE). EE consists of about ten rats housed in a large cage containing various attributes (e.g. running wheels, shelter house, climbing frame, additional nesting material) $[107,108]$. Numerous studies indicate that such an enriched environment induces morphological and molecular changes in the brain. For instance, animals housed in enriched conditions display increased cortical mass $[109,110]$ and neurotransmitter levels $[111,112]$ and perform better on learning and memory tasks compared to rats housed in individual standard cages $[113,114]$. However, with respect to the effects of EE on pain, only few studies are available. Home cage conditions have been demonstrated to significantly affect the magnitude of inflammation-induced nociception [115]. In addition, EE-housing was demonstrated to determine the duration and degree of reversal tactile allodynia in a severe spinal cord injury pain model, during which 
the neurotrophin Brain-Derived Neurotrophic Factor (BDNF) could play a neuroprotective role [116]. Finally, EE has been shown to significantly affect thermal sensitivity, using an operant orofacial pain assay [117].

Besides, the biological mechanism underlying the effect of housing on pain duration and/or magnitude is poorly described. Although studies on environmentally driven plasticity in the CNS have traditionally focused on altered neuronal function, non neuronal cells are also known to be strongly influenced by EE. Astrocyte morphology and number have been reported to change if rats are reared in complex environments, with the changes depending both on the duration of housing as well as on the cortical layer in which the astrocytes reside [118-120]. Changes in astrocyte morphology are known to be correlated to modifications in synapse formation $[121,122]$. Moreover, not only astroglial cells respond to EE but also a reduction of proliferating microglia has been found in EE-housed rats, following a cortical infarct [123]. The microglial response was suggested to favor the better functional outcome in these animals.

Hence, the need of experimental models to further understand and optimize the impact of the environment on postoperative inflammatory pain remains. 


\section{Research questions}

The research described in this thesis is aimed at investigating the effects of the environment on inflammatory pain using a rat model of knee inflammation. These effects were studied with special focus at behavioral recovery. In this thesis, the following research questions were addressed:

1. Can the CatWalk method be used for objective assessment of pain-induced gait changes in a rat model of inflammatory pain?

2. Does housing in an enriched environment enhance the recovery from inflammatory pain?

3. What is the differential effect of the two main components of an enriched environment on the recovery from inflammatory pain: physical versus social enrichment?

In chapters 2 and 3, the CatWalk gait analysis system was studied and tested for use in acute (chapter 2) and chronic (chapter 3) phases in a rat model of knee inflammatory pain. Experimental inflammatory pain was induced into the right knee of the rat through the injection of carrageenan.

In chapter 4, the causality of pain in the CatWalk-gait changes observed in this model of inflammatory pain was demonstrated using an opioid analgesic, the Fentanyl.

Chapters 5, 6 and 7 focus on the EE-induced behavioral recovery after chronic inflammatory pain. In chapter 5 , the effect of an EE housing after the injection of carrageenan into the knee of adult rats was studied. As a first step in understanding the underlying mechanisms involved in EE housing effect on inflammatory pain, the role of astroglial cells was studied. In chapter 6, the effect of EE housing prior to the injection of carrageenan into the knee was studied. In chapter 7, we examined the differential effect on inflammatory pain of the two main components of an EE: social versus physical enrichment. 


\section{References}

1. Nikolajsen, L. and T.S. Jensen, Phantom limb pain. Br J Anaesth, 2001. 87(1): p. 107-16

2. Jung, B.F., et al., Neuropathic pain following breast cancer surgery: proposed classification and research update. Pain, 2003. 104(1-2): p. 1-13

3. Aasvang, E. and H. Kehlet, Chronic postoperative pain: the case of inguinal herniorrhaphy. Br J Anaesth, 2005. 95(1): p. 69-76

4. Gottschalk, A., et al., Preventing and treating pain after thoracic surgery. Anesthesiology, 2006. 104(3): p. $594-600$

5. Bruce, J., et al., The prevalence of chronic chest and leg pain following cardiac surgery: a historical cohort study. Pain, 2003. 104(1-2): p. 265-73

6. Nikolajsen, L., et al., Chronic pain following Caesarean section. Acta Anaesthesiol Scand, 2004. 48(1): p. $111-6$

7. Brandsborg, B., et al., Risk factors for chronic pain after hysterectomy: a nationwide questionnaire and database study. Anesthesiology, 2007. 106(5): p. 1003-12

8. Nikolajsen, L., et al., Chronic pain following total hip arthroplasty: a nationwide questionnaire study. Acta Anaesthesiol Scand, 2006. 50(4): p. 495-500

9. Reuben, S.S., Preventing the development of complex regional pain syndrome after surgery. Anesthesiology, 2004. 101(5): p. 1215-24

10. Kelhet, H., Persistent postsurgical pain: surgical risk factors and strategies for prevention, in Pain: an updated review, R.S. Castro-Lopez J., Schmelz M., Editor. 2008, IASP Press

11. Fanning, N.F., et al., Inhibition of neutrophil apoptosis after elective surgery. Surgery, 1999. 126(3): p. $527-34$

12. Savill, J. and C. Haslett, Granulocyte clearance by apoptosis in the resolution of inflammation. Semin Cell Biol, 1995. 6(6): p. 385-93

13. Lavernia, C., et al., Multimodal pain management and arthrofibrosis. J Arthroplasty, 2008. 23(6 Suppl 1): p. $74-9$

14. Cousins, M.J., I. Power, and G. Smith, 1996 Labat lecture: pain-a persistent problem. Reg Anesth Pain Med, 2000. 25(1): p. 6-21

15. Schaible, H.G. and B.D. Grubb, Afferent and spinal mechanisms of joint pain. Pain, 1993. 55(1): p. 5-54

16. Siddall, P.J. and M.J. Cousins, Introduction to pain mechanisms. Implication for neural blockade., in Neural Blockade in Clinical Anesthesia and Management of Pain, Lippincott-Raven, Editor. 1997: Philadelphia

17. Milligan, E.D. and L.R. Watkins, Pathological and protective roles of glia in chronic pain. Nat Rev Neurosci, 2009. 10(1): p. 23-36

18. Willis, W.D. and R.E. Coggeshall, Sensory mechansims of the spinal cord. 1991, New-York: Plenum

19. Woolf, C.J. and S.W. Thompson, The induction and maintenance of central sensitization is dependent on $N$-methyl-D-aspartic acid receptor activation; implications for the treatment of post-injury pain hypersensitivity states. Pain, 1991. 44(3): p. 293-9

20. Halassa, M.M., T. Fellin, and P.G. Haydon, The tripartite synapse: roles for gliotransmission in health and disease. Trends Mol Med, 2007. 13(2): p. 54-63 
21. Pocock, J.M. and H. Kettenmann, Neurotransmitter receptors on microglia. Trends Neurosci, 2007.30(10): p. 527-35

22. Meller, S.T., et al., The possible role of glia in nociceptive processing and hyperalgesia in the spinal cord of the rat. Neuropharmacology, 1994. 33(11): p. 1471-8

23. Watkins, L.R., et al., Evidence for the involvement of spinal cord glia in subcutaneous formalin induced hyperalgesia in the rat. Pain, 1997.71(3): p. 225-35

24. Hashizume, H., et al., Central administration of methotrexate reduces mechanical allodynia in an animal model of radiculopathy/sciatica. Pain, 2000. 87(2): p. 159-69

25. Ledeboer, A., et al., Minocycline attenuates mechanical allodynia and proinflammatory cytokine expression in rat models of pain facilitation. Pain, 2005. 115(1-2): p. 71-83

26. Reeve, A.J., et al., Intrathecally administered endotoxin or cytokines produce allodynia, hyperalgesia and changes in spinal cord neuronal responses to nociceptive stimuli in the rat. Eur J Pain, 2000. 4(3): p. 24757

27. Oka, T. and T. Hori, Brain cytokines and pain, in Cytokines and pain, L.R. Watkins and S.F. Maier, Editors. 1999: Basel. p. 183-204

28. Raghavendra, V., F. Tanga, and J.A. DeLeo, Inhibition of microglial activation attenuates the development but not existing hypersensitivity in a rat model of neuropathy. J Pharmacol Exp Ther, 2003. 306(2): p. $624-30$

29. Tanga, F.Y., V. Raghavendra, and J.A. DeLeo, Quantitative real-time RT-PCR assessment of spinal microglial and astrocytic activation markers in a rat model of neuropathic pain. Neurochem Int, 2004. 45(2-3): p. 397-407

30. Watkins, L.R., et al., Norman Cousins Lecture. Glia as the «bad guys»: implications for improving clinical pain control and the clinical utility of opioids. Brain Behav Immun, 2007. 21(2): p. 131-46

31. Watkins, L.R. and S.F. Maier, Glia: a novel drug discovery target for clinical pain. Nat Rev Drug Discov, 2003. 2(12): p. $973-85$

32. Fu, K.Y., et al., Microglial reactions after subcutaneous formalin injection into the rat hind paw. Brain Res, 1999. 825(1-2): p. 59-67

33. Raghavendra, V., F.Y. Tanga, and J.A. DeLeo, Complete Freunds adjuvant-induced peripheral inflammation evokes glial activation and proinflammatory cytokine expression in the CNS. Eur J Neurosci, 2004. 20(2): p. 467-73

34. Wong, C.S., et al., Effects of NMDA receptor antagonists on inhibition of morphine tolerance in rats: binding at mu-opioid receptors. Eur J Pharmacol, 1996. 297(1-2): p. 27-33

35. Meert, T.F. and M. De Kock, Potentiation of the analgesic properties of fentanyl-like opioids with alpha 2-adrenoceptor agonists in rats. Anesthesiology, 1994. 81(3): p. 677-88

36. Sivilotti, L. and C.J. Woolf, The contribution of GABAA and glycine receptors to central sensitization: disinhibition and touch-evoked allodynia in the spinal cord. J Neurophysiol, 1994. 72(1): p. 169-79

37. Williams, M.C. and J.J. Ivanusic, Evidence for the involvement of the spinoparabrachial pathway, but not the spinothalamic tract or post-synaptic dorsal column, in acute bone nociception. Neurosci Lett, 2008. 443(3): p. $246-50$ 
38. Jones, C.K., S.C. Peters, and H.E. Shannon, Synergistic interactions between the dual serotonergic, noradrenergic reuptake inhibitor duloxetine and the non-steroidal anti-inflammatory drug ibuprofen in inflammatory pain in rodents. Eur J Pain, 2007. 11(2): p. 208-15

39. Tracey, I. and P.W. Mantyh, The cerebral signature for pain perception and its modulation. Neuron, 2007. 55(3): p. 377-91

40. Sommer, M., et al., Prevalence and predictors of postoperative pain after ear, nose, and throat surgery. Arch Otolaryngol Head Neck Surg, 2009. 135(2): p. 124-30

41. Sinatra, R.S., J. Torres, and A.M. Bustos, Pain management after major orthopaedic surgery: current strategies and new concepts. J Am Acad Orthop Surg, 2002. 10(2): p. 117-29

42. Services, U.D.o.H.a.H., Acute Pain Management: Operative Medical Procedures and Trauma, in Clinical Practices Guidelines No1, P.H.S.A.f.H.C.P.a. Research, Editor. 1992: Rockville, MD

43. Austrup, M.L. and G. Korean, Analgesic agents for the postoperative period. Opioids. Surg Clin North Am, 1999. 79(2): p. $253-73$

44. Pasero, C., R.K. Portenoy, and M. McCaffery, Opioid analgesics, in Pain: Clinical manual, M. McCaffery and C. Pasero, Editors. 1999, Mosby: St Louis, MO

45. Muranyi, M. and Z. Radak, [Pain and opioids]. Orv Hetil, 2008. 149(50): p. 2363-70

46. Boden, B.P., et al., Analgesic effect of intraarticular morphine, bupivacaine, and morphine/bupivacaine after arthroscopic knee surgery. Arthroscopy, 1994. 10(1): p. 104-7

47. Allen, G.C., et al., Postarthroscopy analgesia with intraarticular bupivacaine/morphine. A randomized clinical trial. Anesthesiology, 1993. 79(3): p. 475-80

48. White, A.P., S. Laurent, and D.J. Wilkinson, Intra-articular and subcutaneous prilocaine with adrenaline for pain relief in day case arthroscopy of the knee joint. Ann R Coll Surg Engl, 1990. 72(6): p. 350-2

49. Henderson, R.C., et al., Postarthroscopy analgesia with bupivacaine. A prospective, randomized, blinded evaluation. Am J Sports Med, 1990. 18(6): p. 614-7

50. Ates, Y., et al., Comparison of prilocaine and bupivacaine for post-arthroscopy analgesia: a placebo-controlled double-blind trial. Arthroscopy, 1994. 10(1): p. 108-9

51. Miaskowski, C., Patient-controlled modalities for acute postoperative pain management. J Perianesth Nurs, 2005. 20(4): p. 255-67

52. Feeney, S.L., The relationship between pain and negative affect in older adults: anxiety as a predictor of pain. J Anxiety Disord, 2004. 18(6): p. 733-44

53. Ulrich, R.S., View through a window may influence recovery from surgery. Science, 1984. 224(4647): p. $420-1$

54. Long, R., Healing by design. Eight key considerations for building therapeutic environments. Health Facil Manage, 2001. 14(11): p. 20-2

55. Ananth, S., Building healing spaces. Explore (NY), 2008. 4(6): p. 392-3

56. Lyons, A.S. and R.J. Petrucelli, An Illustrated History. 1987, New-York: Harry N. Abrams

57. Nightingale, F., Sick nursing and health nursing. Summary., in The International Congress of Charities. 1893, Correction and Philantropy: Chicago

58. Grumet, G.W., Pandemonium in the modern hospital. N Engl J Med, 1993. 328(6): p. 433-7 
59. Nilsson, U., The anxiety- and pain-reducing effects of music interventions: a systematic review. Aorn J, 2008. 87(4): p. 780-807

60. Schweitzer, M., L. Gilpin, and S. Frampton, Healing spaces: elements of environmental design that make an impact on health. J Altern Complement Med, 2004. 10 Suppl 1: p. S71-83

61. Fry, W.F., Jr., The physiologic effects of humor, mirth, and laughter. JAMA, 1992.267(13): p. 1857-8

62. Coderre, T.J. and P.D. Wall, Ankle joint urate arthritis (AJUA) in rats: an alternative animal model of arthritis to that produced by Freund's adjuvant. Pain, 1987. 28(3): p. 379-93

63. Rosenthale, M.E., J. Kassarich, and F. Schneider, Jr., Effect of anti-inflammatory agents on acute experimental synovitis in dogs. Proc Soc Exp Biol Med, 1966. 122(3): p. 693-6

64. Santer, V., A. Sriratana, and D.A. Lowther, Carrageenin-induced arthritis: V. A morphologic study of the development of inflammation in acute arthritis. Semin Arthritis Rheum, 1983. 13(2): p. 160-8

65. Schaible, H.G. and R.F. Schmidt, Effects of an experimental arthritis on the sensory properties of fine articular afferent units. J Neurophysiol, 1985. 54(5): p. 1109-22

66. Schumacher, H.R., P. Phelps, and C.A. Agudelo, Urate crystal induced inflammation in dog joints: sequence of synovial changes. J Rheumatol, 1974. 1(1): p. 102-13

67. Okuda, K., et al., Arthritis induced in cat by sodium urate: a possible animal model for tonic pain. Pain, 1984. 18(3): p. 287-97

68. Pearson, C.M., Experimental Joint Disease Observations on Adjuvant-Induced Arthritis. J Chronic Dis, 1963. 16: p. $863-74$

69. Billingham, M.E.J. and G.E. Davies, Experimental models of arthitis in animals as screening tests for drugs to treat arthritis in man, in Handbook of Experimental Pharmacology, G.V.R. Born, et al., Editors. 1979. p. 108-144

70. Puig, S. and L.S. Sorkin, Formalin-evoked activity in identified primary afferent fibers: systemic lidocaine suppresses phase-2 activity. Pain, 1996. 64(2): p. 345-55

71. McNamara, C.R., et al., TRPA1 mediates formalin-induced pain. Proc Natl Acad Sci U S A, 2007. 104(33): p. $13525-30$

72. Winter, C.A., E.A. Risley, and G.W. Nuss, Carrageenin-induced edema in hind paw of the rat as an assay for antiiflammatory drugs. Proc Soc Exp Biol Med, 1962. 111: p. 544-7

73. Radhakrishnan, R., S.A. Moore, and K.A. Sluka, Unilateral carrageenan injection into muscle or joint induces chronic bilateral hyperalgesia in rats. Pain, 2003. 104(3): p. 567-77

74. Mense, S., [Neurobiological mechanisms of muscle pain referral.]. Schmerz, 1993. 7(4): p. 241-9

75. Schaible, H.G., A. Ebersberger, and G.S. Von Banchet, Mechanisms of pain in arthritis. Ann N Y Acad Sci, 2002. 966: p. 343-54

76. Di Rosa, M., J.P. Giroud, and D.A. Willoughby, Studies on the mediators of the acute inflammatory response induced in rats in different sites by carrageenan and turpentine. J Pathol, 1971. 104(1): p. 15-29

77. Nantel, F., et al., Distribution and regulation of cyclooxygenase-2 in carrageenan-induced inflammation. Br J Pharmacol, 1999. 128(4): p. 853-9

78. Hay, C. and J. de Belleroche, Carrageenan-induced hyperalgesia is associated with increased cyclo-oxygenase-2 expression in spinal cord. Neuroreport, 1997. 8(5): p. 1249-51 
79. Schaible, H.G., et al., Release of immunoreactive substance $P$ in the spinal cord during development of acute arthritis in the knee joint of the cat: a study with antibody microprobes. Brain Res, 1990. 529(1-2): p. 214-23

80. Sluka, K.A. and K.N. Westlund, An experimental arthritis in rats: dorsal horn aspartate and glutamate increases. Neurosci Lett, 1992. 145(2): p. 141-4

81. Sorkin, L.S., et al., Neural changes in acute arthritis in monkeys. IV. Time-course of amino acid release into the lumbar dorsal horn. Brain Res Rev, 1992. 17(1): p. 39-50

82. Yang, L.C., M. Marsala, and T.L. Yaksh, Characterization of time course of spinal amino acids, citrulline and PGE2 release after carrageenan/kaolin-induced knee joint inflammation: a chronic microdialysis study. Pain, 1996. 67(2-3): p. 345-54

83. Rivot, J.P., J. Montagne-Clavel, and J.M. Besson, Subcutaneous formalin and intraplantar carrageenan increase nitric oxide release as measured by in vivo voltammetry in the spinal cord. Eur J Pain, 2002.6(1): p. $25-34$

84. Garry, M.G. and K.M. Hargreaves, Enhanced release of immunoreactive CGRP and substance P from spinal dorsal horn slices occurs during carrageenan inflammation. Brain Res, 1992. 582(1): p. 139-42

85. Sluka, K.A. and K.N. Westlund, Behavioral and immunohistochemical changes in an experimental arthritis model in rats. Pain, 1993. 55(3): p. 367-77

86. Schaible, H.G., R.F. Schmidt, and W.D. Willis, Enhancement of the responses of ascending tract cells in the cat spinal cord by acute inflammation of the knee joint. Exp Brain Res, 1987. 66(3): p. 489-99

87. Dougherty, P.M. and W.D. Willis, Enhanced responses of spinothalamic tract neurons to excitatory amino acids accompany capsaicin-induced sensitization in the monkey. J Neurosci, 1992. 12(3): p. 883-94

88. Hoheisel, U., B. Sander, and S. Mense, Blockade of nitric oxide synthase differentially influences background activity and electrical excitability in rat dorsal horn neurones. Neurosci Lett, 1995. 188(2): p. 143-6

89. Pertovaara, A., A neuronal correlate of secondary hyperalgesia in the rat spinal dorsal horn is submodality selective and facilitated by supraspinal influence. Exp Neurol, 1998. 149(1): p. 193-202

90. Urban, M.O., P.K. Zahn, and G.F. Gebhart, Descending facilitatory influences from the rostral medial medulla mediate secondary, but not primary hyperalgesia in the rat. Neuroscience, 1999. 90(2): p. 349-52

91. Le Bars, D., M. Gozariu, and S.W. Cadden, Animal models of nociception. Pharmacol Rev, 2001. 53(4): p. $597-652$

92. Chaplan, S.R., et al., Quantitative assessment of tactile allodynia in the rat paw. J Neurosci Methods, 1994. 53(1): p. 55-63

93. Dixon, W.J., Efficient analysis of experimental observations. Annu Rev Pharmacol Toxicol, 1980. 20: p. 441-62

94. Tumati, S., et al., Intrathecal Raf-1-selective siRNA attenuates sustained morphine-mediated thermal hyperalgesia. Eur J Pharmacol, 2008. 601(1-3): p. 207-8

95. Hargreaves, K., et al., A new and sensitive method for measuring thermal nociception in cutaneous hyperalgesia. Pain, 1988. 32(1): p. 77-88

96. Marcinkiewcz, C.A., et al., Social defeat stress potentiates thermal sensitivity in operant models of pain processing. Brain Res, 2008 
97. LaGraize, S.C., et al., Selective regulation of pain affect following activation of the opioid anterior cingulate cortex system. Exp Neurol, 2006. 197(1): p. 22-30

98. Vierck, C.J., et al., Low dose systemic morphine attenuates operant escape but facilitates innate reflex responses to thermal stimulation. J Pain, 2002. 3(4): p. 309-19

99. Bech, P., Health-related quality of life measurements in the assessment of pain clinic results. Acta Anaesthesiol Scand, 1999. 43(9): p. 893-6

100. Min, S.S., et al., A novel method for convenient assessment of arthritic pain in voluntarily walking rats. Neurosci Lett, 2001.308(2): p. 95-8

101. Coulthard, P., S.U. Simjee, and B.J. Pleuvry, Gait analysis as a correlate of pain induced by carrageenan intraplantar injection. J Neurosci Methods, 2003. 128(1-2): p. 95-102

102. Gorska, T., H. Majczynski, and W. Zmyslowski, Overground locomotion in intact rats: contact electrode recording. Acta Neurobiol Exp (Wars), 1998. 58(3): p. 227-37

103. Koopmans, G.C., et al., Strain and locomotor speed affect over-ground locomotion in intact rats. Physiol Behav, 2007.92(5): p. 993-1001

104. Koopmans, G.C., et al., Circulating insulin-like growth factor I and functional recovery from spinal cord injury under enriched housing conditions. Eur J Neurosci, 2006. 23(4): p. 1035-46

105. Hamers, F.P., G.C. Koopmans, and E.A. Joosten, CatWalk-Assisted Gait Analysis in the Assessment of Spinal Cord Injury. J Neurotrauma, 2006. 23(3-4): p. 537-48

106. Vrinten, D.H. and F.F. Hamers, 'CatWalk' automated quantitative gait analysis as a novel method to assess mechanical allodynia in the rat; a comparison with von Frey testing. Pain, 2003. 102(1-2): p. 203-9

107. Lankhorst, A.J., et al., Effects of enriched housing on functional recovery after spinal cord contusive injury in the adult rat. J Neurotrauma, 2001. 18(2): p. 203-15

108. Koopmans, G.C., et al., The assessment of locomotor function in spinal cord injured rats: the importance of objective analysis of coordination. J Neurotrauma, 2005. 22(2): p. 214-25

109. Rosenzweig, M.R., et al., Effects of environmental complexity and training on brain chemistry and anatomy: a replication and extension. J Comp Physiol Psychol, 1962. 55: p. 429-37

110. Bennett, E.L., M.R. Rosenzweig, and M.C. Diamond, Rat brain: effects of environmental enrichment on wet and dry weights. Science, 1969. 163(869): p. 825-6

111. O'Shea, L., et al., Neonatal 6-hydroxydopamine attenuates the neural and behavioral effects of enriched rearing in the rat. Eur J Pharmacol, 1983. 92(1-2): p. 43-7

112. Naka, F., et al., An enriched environment increases noradrenaline concentration in the mouse brain. Brain Res, 2002. 924(1): p. 124-6

113. Kobayashi, S., Y. Ohashi, and S. Ando, Effects of enriched environments with different durations and starting times on learning capacity during aging in rats assessed by a refined procedure of the Hebb-Williams maze task. J Neurosci Res, 2002. 70(3): p. 340-6

114. Mohammed, A.K., et al., Environmental influence on behaviour and nerve growth factor in the brain. Brain Res, 1990. 528(1): p. 62-72

115. Tall, J.M., Housing supplementation decreases the magnitude of inflammation-induced nociception in rats. Behav Brain Res, 2009. 197(1): p. 230-3 
116. Berrocal, Y., et al., Social and environmental enrichment improves sensory and motor recovery after severe contusive spinal cord injury in the rat. J Neurotrauma, 2007. 24(11): p. 1761-72

117. Rossi, H.L. and J.K. Neubert, Effects of environmental enrichment on thermal sensitivity in an operant orofacial pain assay. Behav Brain Res, 2008. 187(2): p. 478-82

118. Markham, J.A. and W.T. Greenough, Experience-driven brain plasticity: beyond the synapse. Neuron Glia Biol, 2004. 1(4): p. 351-363

119. Sirevaag, A.M. and W.T. Greenough, Differential rearing effects on rat visual cortex synapses. III. Neuronal and glial nuclei, boutons, dendrites, and capillaries. Brain Res, 1987.424(2): p. 320-32

120. Sirevaag, A.M. and W.T. Greenough, Plasticity of GFAP-immunoreactive astrocyte size and number in visual cortex of rats reared in complex environments. Brain Res, 1991. 540(1-2): p. 273-8

121. Jones, T.A. and W.T. Greenough, Ultrastructural evidence for increased contact between astrocytes and synapses in rats reared in a complex environment. Neurobiol Learn Mem, 1996. 65(1): p. 48-56

122. Ullian, E.M., et al., Control of synapse number by glia. Science, 2001. 291(5504): p. 657-61

123. Keiner, S., et al., Rehabilitative therapies differentially alter proliferation and survival of glial cell populations in the perilesional zone of cortical infarcts. Glia, 2008. 56(5): p. 516-27 



\section{The CatWalk method:}

a detailed analysis of behavioral

\section{changes after acute inflammatory}

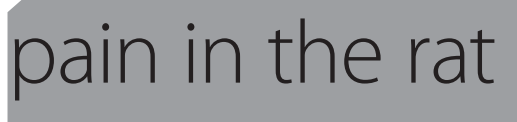

A.F. Gabriel ${ }^{1,3}$, M.A.E. Marcus', W.M.M. Honig'1, G.H.I.M.

Walenkamp², E.A.J. Joosten ${ }^{1,3}$

'Department of Anesthesiology, Academic Hospital Maastricht, 6200MD Maastricht, the Netherlands

2Department of Surgery and Orthopedics, Academic Hospital Maastricht, 6200MD Maastricht, the Netherlands

${ }^{3}$ European Graduate School of Neuroscience (EURON), University of Maastricht, P.O. Box 616, 6200 MD, Maastricht, The Netherlands

Journal of Neuroscience Methods, 163 (2007), 9-16 


\section{Abstract}

Experimental pain research is often complicated by the absence of an objective and detailed method to analyze behavioral changes. In the present study acute pain was induced into the right knee of the rat $(n=15)$ through the injection of $2 \mathrm{mg}$ carrageenan (CAR) in saline. A control group received vehicle injection into the knee $(\mathrm{n}=15)$. With the use of an automated quantitative gait analysis system, the CatWalk, it was possible to quantitatively analyze behavioral changes at post-injection time 2.5, 4, 24 and 48 hours. The CatWalk analysis of individual paw parameters like the intensity of the paw print or the time contact with the floor showed a significant effect after CAR injection into the knee. These CatWalk parameters were highly correlated with von Frey data and thus representative for the development of mechanical allodynia. Furthermore, detailed CatWalk analysis of the gait (i.e coordinated interaction between left and right hindlimb) showed very fine, accurate and significant coordination changes in the experimental rats as of 4 hours post-injection. In conclusion, the CatWalk method allows an objective and detailed detection of both pain induced gait adaptations as well as the development of mechanical allodynia in an acute inflammatory pain model. 


\section{Introduction}

One of the main problems in experimental pain studies is the objective assessment of pain. Due to the absence of verbal communication in animals, pain analysis needs to be based on either physiological or behavioral analysis, the last one being described as the most specific [1]. Many experimental models for acute and chronic pain have been developed. Within these models, pain is generally assessed using a variety of tests which almost exclusively are based on the response to paininducing actions. For assessment of mechanical allodynia the most common test used is the von Frey $[2,3]$. The von Frey test has several disadvantages, like it is based on the investigators interpretation, the definition of withdrawal threshold, and the bending force of the filaments can be influenced by ambient humidity and temperature.

Recently the CatWalk has been suggested as a method which allows objective assessment of mechanical allodynia in a model of chronic pain [4]. The computerassisted method of locomotor analysis allows the rapid quantification of individual paw parameters as well as parameters related to interlimb coordination [5]. In the context of experimental pain research, the CatWalk might become even more valuable because it allows a complete and detailed analysis of how the animal handles pain during normal behavior, like gait. The latter is relevant because in the clinical situation pain is generally evaluated based on how patients handle pain during normal life [6]. All in all, various initial attempts have thus recently been undertaken to partly analyse gait changes in an experimental model of acute pain $[7,8]$. Although the techniques described previously give valuable information about gait changes in pain models, the CatWalk technique we use here is the first one allowing a fast and objective measure of both numerous static and dynamic gait related parameters. Hence, the present study aims to demonstrate that the CatWalk method allows an automated and complete analysis of gait related changes after a carrageenan induced-acute pain. In addition, we hypothesize that CatWalk parameters related to individual paws can be used to assess mechanical allodynia in this acute experimental pain model.

In the present study, the various behavioral parameters analysed were classified into two groups: first, a group of parameters related to pain induced-gait adaptations (based on CatWalk parameters) and second, parameters related to mechanical allodynia (based on von Frey and CatWalk parameters). 


\section{Materials and methods}

\section{Animals}

A total of 30 male Sprague-Dawley rats weighing 250-300 g were used. During the experiment, animals were housed individually under standard conditions with a $12 \mathrm{~h} / 12 \mathrm{~h}$ light/dark cycle. Water was available ad libitum. Starting two weeks prior the injection, the rats were restricted for the food (with 12-15 $\mathrm{g}$ of food per animal per day). This study was approved by the Ethics Committee on Animal Experiments of the University of Maastricht (DEC protocol no.2005/096).

\section{Experimental design}

Prior to injection ( $\mathrm{t}=-1$ day), CatWalk and von Frey tests were performed in order to determine the baseline. The experimental group $(n=15)$ was injected intra-articularly, at $\mathrm{t}=0$, with $2 \mathrm{mg}$ of $\lambda$-carrageenan $(200 \mu \mathrm{l})$ (Sigma-Aldrich, no C3889) into the knee joint of the right hind paw under isofluorane $(2.5 \%)$ anaesthesia. The control-group $(\mathrm{n}=15)$ was injected intra-articularly with a saline solution $(200 \mu \mathrm{l})$ into the same joint. Von Frey test of both ipsilateral and contralateral paws and CatWalk analysis were performed at $\mathrm{t}=2.5 \mathrm{~h}, 4 \mathrm{~h}, 24 \mathrm{~h}$ and $48 \mathrm{~h}$ post-injection. Animals were sacrificed with an overdose of Nembutal (150 mg/kg, intraperitoneal injection).

\section{Assessment of pain induced-gait adaptation with Cat- Walk method}

Detailed analysis of gait was performed on walking rats using the CatWalk method [9-11]. Briefly, light from a fluorescent tube is sent through a glass plate. Light rays are completely reflected internally. As soon as anything, e.g. a rat's paw, is in contact with the glass surface, light is reflected downwards. It results in a sharp image of a bright paw print. The whole run is recorded via a camera placed under the glass plate. Two weeks prior to the injection, rats were trained to cross the walkway. This training results in crossing the pathway without hesitation at a constant speed. Sweet pallets (Noyes Precision pellets PJPPP-0045; Sandown Chemical Ltd., Hampton, United Kingdom) are placed at the end of the walkway. Animals are motivated via a $12 \mathrm{~g} /$ day food restriction protocol.

In the present study, parameters related to single paws were analyzed:

- Intensity of the paw print (expressed in arbitrary units (a.u.)): this parameter describes the mean pressure exerted by one individual paw during the floor 
contact, during the whole crossing of the walkway. Data analysis was performed with a threshold value of 40 (arbitrary units, possible range 0-225), i.e. all pixels brighter than 40 are used.

- Duty factor (expressed in\%): The duty factor represents the ratio between the stance duration and the stepcycle duration. It is calculated according to the formula: stance phase duration/[stance + swing phases duration] ${ }^{\star} 100$ [12], where the stance duration is the time of contact of one paw with the floor during one single stepcycle and the swing duration is the time of non-contact with the floor during the stepcycle. The time contact (or stance phase duration) of one paw can be considered as directly related to the perceived pain in the limb, as the duty factor therefore is. According to its definition, this parameter can not be defined if the paw is not placed on the floor.

- Base of support of the hind limbs (BOS HL) (expressed in $\mathrm{mm}$ ): The base of support of the hind limbs is the distance between the two hind paws of the rat. It is measured for each stepcycle and an average is calculated per run. Therefore, this parameter cannot be measured if one of the two hind paws is not in contact with the floor during the run.

- Relative paw placement (expressed in $\mathrm{mm}$ ): This parameter describes the distance between the placement of the fore and hind paws of the same side of the animal, at each stepcycle. A mean is calculated for the entire run. A negative value implicates that the hind paw is placed upward compared to the fore paw. Thus a positive relative paw placement implicates that the hind paw is placed backward compared to the fore paw. Subsequently, if the animal does not use one of its paws, this parameter can not be measured for the corresponding side.

- Maximum area (expressed in $\mathrm{mm}^{2}$ ): The maximal contact area describes the paw area contacted at the moment of maximal paw-floor contact, during stance (Joosten et al., 2004).

- Print area (expressed in $\mathrm{mm}^{2}$ ): This parameter describes the total floor area contacted by the paw during the stance phase [13].

- Box width (expressed in $\mathrm{mm}$ ): it is a measure of the width of the print area. This parameter can be considered as related to the development of hyperalgesia or allodynia in the limb.

- Box length (expressed in $\mathrm{mm}$ ): it is a measure of the length of the print area. This parameter can also be considered as related to the development of hyperalgesia or allodynia in the limb.

- Swing speed (expressed in $\mathrm{m} / \mathrm{s}$ ): this parameter is computed from stride length and swing duration.

- Stepcycle duration (expressed in s): it represents the time between two consecutive paw placements (stance + swing duration). 
- Stride length (expressed in $\mathrm{mm}$ ): it is the distance between two consecutive paw placements.

Parameters related to the interlimb coordination were collected as well:

- The regularity index (RI) (expressed in \%): The regularity index (RI) is a measure of interlimb coordination using the analysis of the step sequences [4], according to the formula: $R I=(N S S P \times 4 / P P) \times 100 \%$. NSSP represents the number of normal step sequence patterns and PP the total number of paw placements. Therefore, the RI grades the degree of coordination and represents the percentage of normal step sequence patterns. When step patterns that are not normal are counted, they consist of fewer paw placements than 4 , and are thus counted each and every one (PP). Both extra paw placements and loss of paw placements (irregular walking on three paws) will decrease the Regularity Index (Vrinten and Hamers, 2003). If a rat has no normal step sequence (i.e. does not make use of the injected paw), the RI is $0 \%$.

- The average phase lag and the phase lag variability (expressed in \%): a recently described parameter about interlimb coordination using the timed relationships between footfalls of two different paws [5]. The phase lag relies the initial contact of one paw (the Target) to the stride cycle of another paw (the Anchor) and is expressed in percentage. The phase lags are calculated for every single stride cycle. When a phase lag exceeds $100 \%$, an error is scored and the value is discarded. To cross the walkway, the animal typically needs five to six stride cycles, from which the average phase lag is calculated, as well as the phase lag variability (SD of the mean). The phase lag variability is therefore a measure of the accuracy in the interlimb coordination. The phase lag within girdle pairs is on average $50 \%$ (the initial contact of one limb falls about halfway in the stride cycle of the contralateral paw) and is independent of the speed [5]. Locomotor activity between limbs is defined as coordinated when the phase lag variability is within the $95 \%$ confidence interval of pre-operative measurement (Koopmans et al., 2006). In this study, we exclusively focus on the pelvic girdle pairs, considering once the left paw as the target and once the right paw as the target. As these parameters are based on the use of four paws, if one of them is not used, these parameters can not be measured for that specific paw.

\section{Assessment of mechanical allodynia with the von Frey test}

Paw withdrawal threshold in response to a mechanical stimulus was determined using a series of filaments of varying thickness. Animals were placed in a plastic cage with a metal mesh floor, allowing them to move freely. For approximately $10 \mathrm{~min}$ prior to testing, they were placed in this cage, to allow them to acclimatize to this environment. Von Frey filaments were applied to the mid-plantar surface of both hind paws, 
one paw a time, through the mesh floor. We used 14 Touched-Test von Frey filaments, number 5 to 16 (North Coast Medical, Inc.Ca. USA) with a regularly calibrated stiffness corresponding to $0.16,0.4,0.6,1.0,1.4,2.0,4.0,6.0,8.0,10,15,26,60$ and 100 grams. Probing was only performed when the animal's four paws were in contact with the floor. Each probe was applied to the foot until it just bent. A minimum of three withdrawals of the tested paw out of 5 filament applications was considered as a positive response. Filaments were applied in ascending order, and the smallest filament that elicited a positive response was considered the threshold stimulus. In this test, the contralateral paw acts as control compared to the injected paw.

\section{Statistics}

Comparisons between Control and CAR groups were made by the means of multiple ways-ANOVA tests (repeated measures), followed by a Bonferonni correction $[\mathrm{p}<0.05$ ]. Comparison with the pre-operative values within one group have been statistically tested via a paired t-test $[\mathrm{p}<0.01]$.

To analyze the possible correlations between the von Frey results and the results obtained with the CatWalk method, a Pearson correlation test was performed. The Pearson correlation coefficient (p.c.c.) was used to study a possible correlation between the von Frey results and the CatWalk parameters related to a single paw. All data are expressed as mean \pm s.e.m.

\section{Results}

No complication after injection of $2 \mathrm{mg}$ of carrageenan in the right knee of the rats was noted $(n=30)$. All 30 animals stayed alive until the end of the experiment. Four rats from the carrageenan group were excluded from a part of the CatWalk analysis. The exclusion was due to the fact that, after the carrageenan injection, these rats used only three paws during crossing of the CatWalk pathway and thus the following parameters could not correctly be assessed in these animals: BOS HL, Duty factor, Phase lag and Phase lag variability and relative paw placement (see definitions in Material and Methods section).

\section{Development of pain induced-gait adaptations: CatWalk test}

The locomotor speed is computed in dividing the length of the walkway by the duration of the run. In the present study, it ranged between $70-80 \mathrm{~cm} / \mathrm{s}$ and did not differ significantly between the two groups. 


\section{Parameters related to single paws:}

Intensity of the paw prints (CatWalk analysis):

The pre-operative value of the intensity from the ipsilateral paw print in the carrageenan group was $71.7 \pm 2.5$ a.u. (arbitrary units). As illustrated in the Figures 1 and $2 \mathrm{~A}$, the carrageenan injection provokes an overall decrease of this intensity $\left(\mathrm{F}_{1,13}=6.256 ; \mathrm{p}<0.05\right)$, down to $52.8 \pm 5.7$ a.u., at 48 hours. No significant difference is observed over time in the intensity of the contralateral paw print. No significant difference was noted in the intensity of the paw prints from the control group.

\section{Duty factor (Catwalk analysis):}

In the carrageenan (CAR) group, the pre-operative value of the duty factor is $45.8 \pm 1.7 \%$ for the ipsilateral paw and $45.7 \pm 1.5 \%$ for the contralateral paw. The carrageenan injection induces an overall increase in the duty factor of the unaffected (contralateral) paw $\left(\mathrm{F}_{5,9}=6.909 ; \mathrm{p}<0.05\right)$, up to $57.7 \pm 1.8 \%$ at $48 \mathrm{~h}$. It is already statistically different from the baseline at 4 hours post-injection (Figures 1 and $2 \mathrm{~B}$ ). This increase represents a longer time contact with the floor (stance phase) as well as a shorter swing phase duration. Whereas in the affected (ipsilateral) paw, the duty factor shows a decrease down to $38.0 \pm 2.8 \%$ at 24 hours after injection $\left(\mathrm{F}_{5,9}=2.996\right.$; $\mathrm{p}<0.05$ ). This decrease represents a shorter time contact with the floor (stance duration) as well as a longer swing phase duration of the injured paw. The duty factor of both hindpaws in the control group shows no detectable difference.

\section{The base-of-support of the hind limbs (BOS HL):}

The pre-operative value of BOS HL in the carrageenan group was $19.2 \pm 0.6 \mathrm{~mm}$ and in the control groups was $20.1 \pm 0.8 \mathrm{~mm}$ (not shown). Injection of carrageenan did not result in an effect on BOS HL during the experiment. No difference between the carrageenan group and the control group has been measured.

\section{The relative paw placement:}

Starting with a pre-operative value of $-6.55 \pm 1.34 \mathrm{~mm}$, no difference in the relative paw placement of the contralateral paws between the carrageenan group and the control was measured (Figure $2 \mathrm{C}$ ). With a pre-operative value of $-7.7 \pm 1.4 \mathrm{~mm}$, no difference was noted over time in the relative paw placement of the ipsilateral paws of the control group. With a pre-operative value of $-5.3 \pm 1.7 \mathrm{~mm}$, the injection of 
carrageenan induced a significant shift up to $104 \pm 35 \%$ backward in the position of the hind-ipsilateral paw compare to the fore-ipsilateral paw $\left(\mathrm{F}_{5,10}=9.665 ; \mathrm{p}<0.005\right)$ (Figure 2D).

The results for the other single paws related CatWalk parameters (swing speed, max. area, print area, box width, box length) were also significantly affected in the CAR injected (data not shown). These parameters were only included in this paper because of the strong correlation found with the von Frey data.

\section{Parameters related to interlimb coordination:}

The phase lag:

If defining the right hind paw as the target, our results show an overall delay (up to $48 \%$ ) in the placement of the ipsilateral paw within the stepcycle of the contralateral paw in the carrageenan group as compared to the control group $\left(\mathrm{F}_{1,19}=27.508\right.$; $\mathrm{p}<0.05$ ) (Figures 1 and 3A). If defining the left paw as the target, a premature use (up to $49 \%$ ) of the contralateral paw compare to the stepcycle duration of the ipsilateral paw is observed in the carrageenan group as compared to the control group $\left(\mathrm{F}_{1,19}=29.297 ; \mathrm{p}<0.05\right)$ (Figures 1 and $3 \mathrm{~B}$ ).

The phase lag variability:

No significant difference in phase lag variability was observed over time, using the right and the left hind paw as the target respectively (Figures $3 \mathrm{C}$ and 3D). A constant phase lag variability represents a constant accuracy in interlimb coordination.

The regularity index (RI):

The pre-operative value for both the carrageenan and the control groups were $96.9 \pm 0.6 \%$ and $98.1 \pm 0.5 \%$ respectively, and did not differ over time. Injection of carrageenan into the right knee did not result in significant changes in the percentage of normal step sequence patterns, with respect to the RI (not shown).

\section{Development of mechanical allodynia: von Frey test}

The mean pre-injection withdrawal threshold was $53.1 \pm 5.5 \mathrm{~g}$. The intra-articular injection of carrageenan in the rat right knee produced an overall decrease of the mechanical withdrawal threshold of the ipsilateral (right) paw $\left(\mathrm{F}_{5,9}=16.518 ; \mathrm{p}<0.05\right)$. 


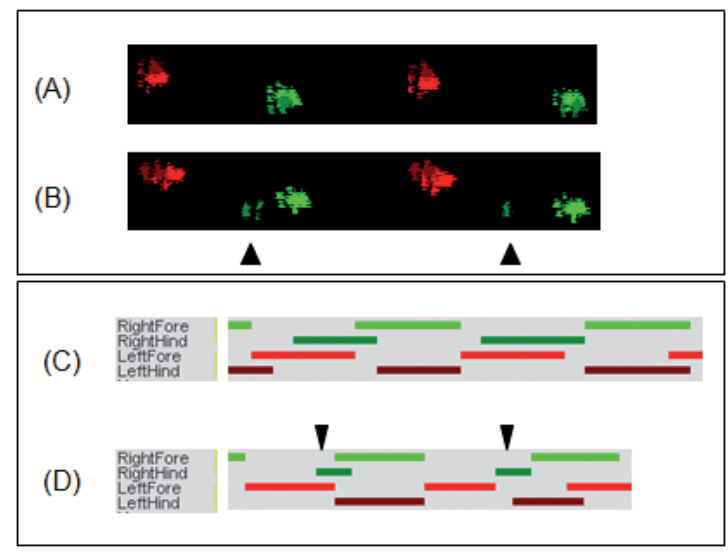

Figure 1: Illustration of CatWalk foot prints of a walking rat $24 \mathrm{~h}$ after (A) a saline injection or (B) a carrageenan injection into the right knee. Decreases in the intensity, the print area as well as in the box length and width can be noted (arrows). Illustration of a CatWalk gait diagram of a walking rat $24 \mathrm{~h}$ after (C) a saline injection or (D) a carrageenan injection into the right knee. Indicated are the paw-floor contacts for each of the four paws over time. The length of each bar represents the duration of the stance phase for that particular paw. The space between bars represents the duration of the swing phase. Decreases in the stance phase as well as a delay in the use of the injured paw are observed (arrows).

This decrease is significant from the baseline already at $2.5 \mathrm{~h}$ post injection (Figure 4 ). A maximal reduction in withdrawal threshold was noted after 4 hours $(6.9 \pm 4.5 \mathrm{~g})$ and this remains stable at this level up to 48 hours (decrease of $83 \pm 13 \%$ ). No significant effect has been observed in the contralateral paw. No effect was observed between the paws of the control animals over time (results not shown).

\section{Correlation between the von Frey test and CatWalk parameters}

Strong correlations between the von Frey test and various CatWalk parameters related to the right hind (affected) paw are shown (Table I). The strongest correlation is observed between von Frey withdrawal threshold and the intensity of the ipsilateral paw print (p.c.c. $=0.94)$.

\section{Discussion}

The present study demonstrates that CatWalk allows an automated, fast, objective and complete analysis of gait related changes after acute pain. The study of CatWalk parameters depicts an accurately coordinated adaptation of the gait to 

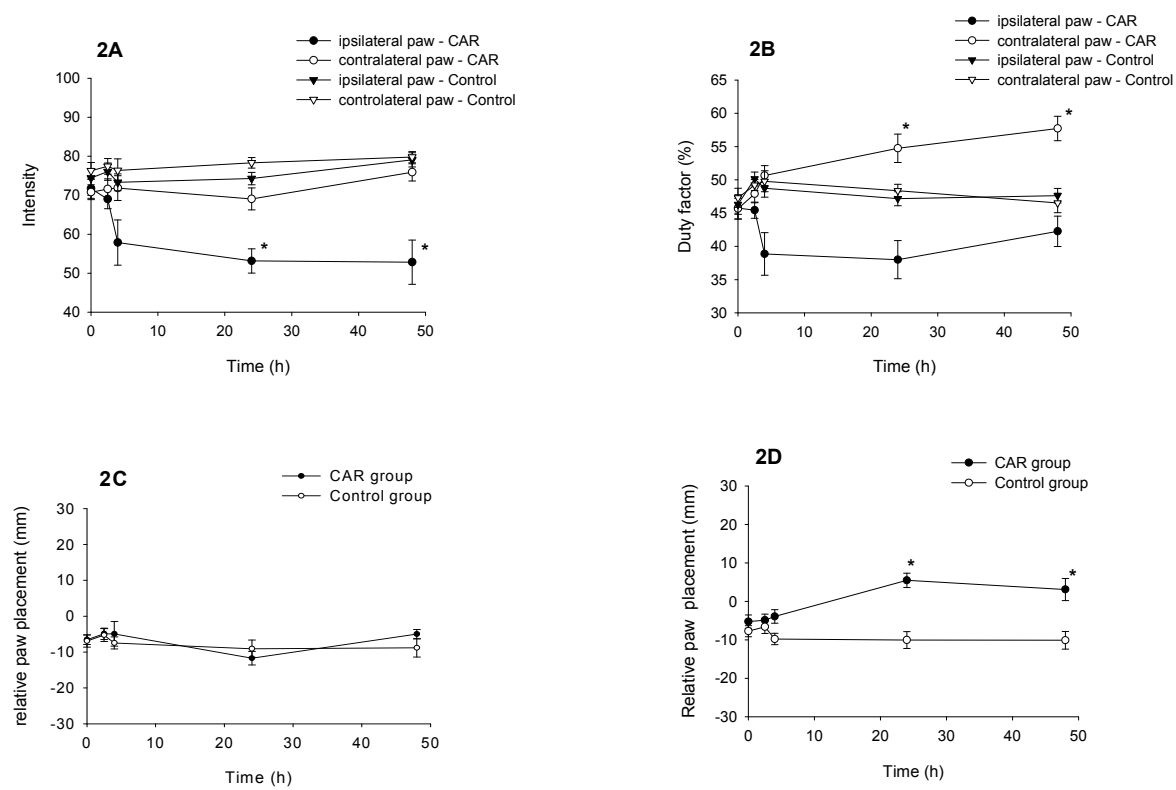

Figure 2: CatWalk analysis: time course of the intensity of the paw printing (2A), the duty factor (2B) and the relative paw placement of the left (2C) and of the right side (2D) analyzed during spontaneous deambulation of the rat. Measurements were taken pre-operatively and at 2.5, 4, 24 and 48 hours post-injection. Data are presented as mean \pm s.e.m.. The symbol $\left(^{*}\right)$ represents values significantly different from the pre-operative value (paired t-test; $p<0.01$ ).

the carrageenan induced inflammation. Besides, CatWalk parameters related to single paws are strongly correlated to the development of mechanical allodynia as assessed by the von Frey withdrawal threshold. The CatWalk is an automated quantitative gait analysis, originally developed for the objective assessment of functional changes in gait after spinal cord injury $[5,9,13,14]$. The computer-assisted method of locomotor analysis allows the rapid quantification of individual parameters as well as parameters related to interlimb coordination [5]. A strong correlation was shown between the development of mechanical allodynia in rats subjected to chronic constriction injury (CCI) as evaluated with von Frey or with three different CatWalk paw parameters: the intensity of paw print, the stance and the swing phase duration of the affected paw [4]. It therefore was concluded that the CatWalk can be used to objectively assess mechanical allodynia in a CCI model of chronic pain. In this study the carrageenan (CAR)-induced inflammation into the knee is used. CAR-induced inflammation into the paw 

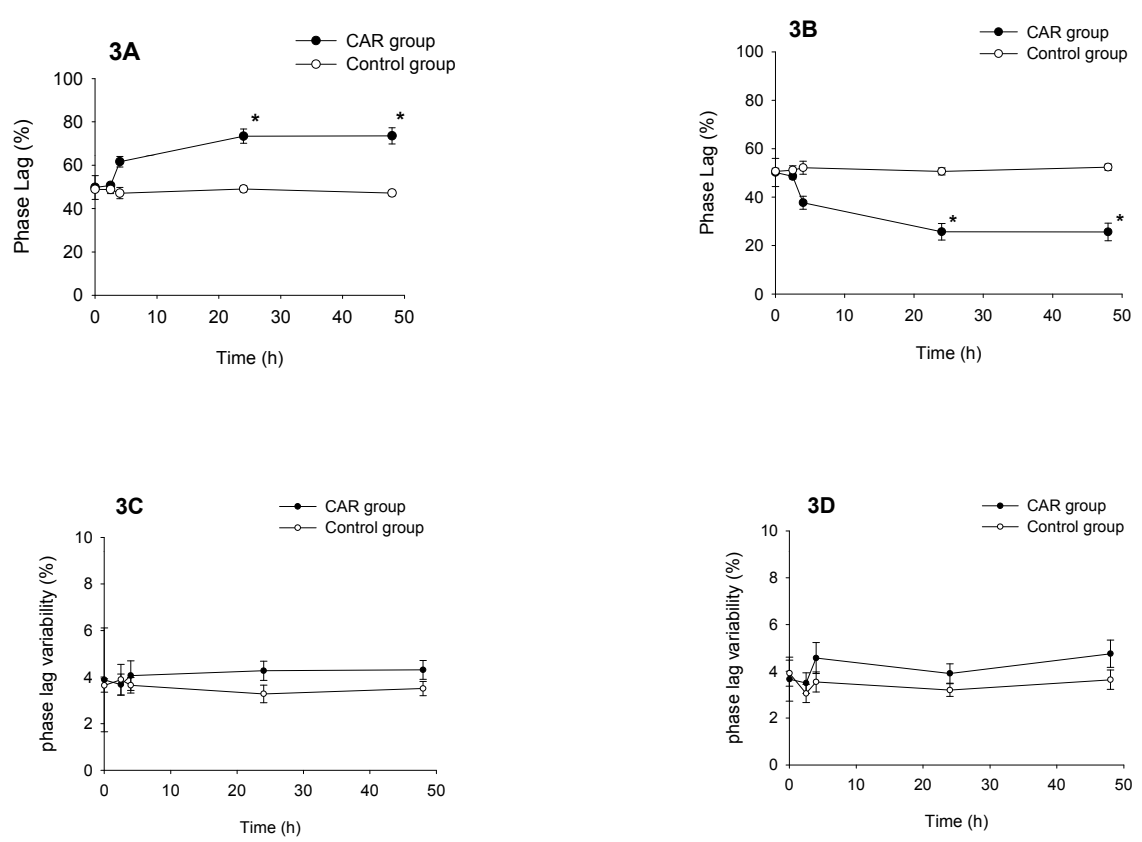

Figure 3: Study over time of different CatWalk parameters, following a carrageenan (CAR group) or a saline solution (Control group) injection: (3A) Phase Lag [target: right paw]; (3B) Phase lag [target: left paw]; (3C) Phase lag variability [target: right paw]: (3D) Phase lag variability [target: left paw]. Data are presented as mean \pm s.e.m.. Measurements were taken pre-operatively and at 2.5, 4, 24 and 48 hours post-injection. The symbol $\left(^{*}\right)$ represents values significantly different from the pre-operative value (paired t-test; $p<0.01$ ).

or the knee is one of the most commonly used experimental models for acute pain [15]. CAR-induced inflammation as originally described by Winter (1962), is acute, nonimmune and highly reproducible [16]. According to Radhakrishnan et al. (2003), the observed histological changes as well as the development of hyperalgesia are directly related to the concentration of carrageenan that is used: $0.3 \%(100 \mu \mathrm{l} ; 0.3 \mathrm{mg})$ of CAR into the knee has no effect, whereas a concentration of $1 \%(100 \mu \mathrm{l} ; 1 \mathrm{mg})$ of CAR produces a short-lasting hyperalgesia that remains ipsilateral [17]. A concentration of $3 \%(100 \mu \mathrm{l} ; 3 \mathrm{mg})$ CAR result in contralateral spreading of the hyperalgesia.

Beside the concentration of the solution, the volume of injection is also an important issue in producing inflammation. As reported by Lam and Ferrell (1993), we injected a total volume of $200 \mu \mathrm{l}$ [18]. However, inducing arthritis through injection of an induction agent into the knee joint has also been performed by many others, using various injected volumes (e.g. [7]; [19]). 


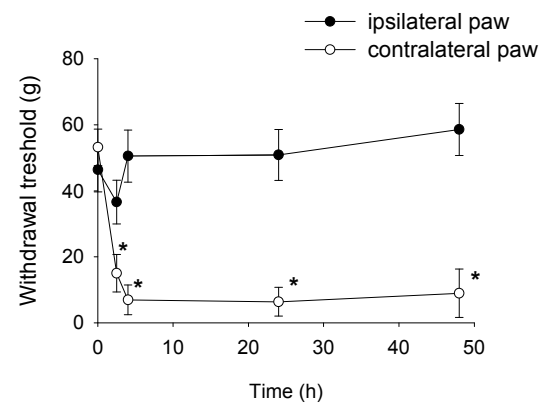

Figure 4: von Frey analysis: time course of withdrawal thresholds to mechanical stimulus following an injection of $1 \%$ carrageenan into the right knee. Thresholds expressed in grams. Measurements were taken preoperatively and at 2.5, 4, 24 and 48 hours post-injection. Data are presented as mean \pm s.e.m.. The symbol $\left(^{*}\right)$ represents values significantly different from the pre-operative value (paired t-test; $p<0.01$ ).

\section{Development of pain-induced gait adaptations}

It is obvious that pain will induce behavioral adaptations. In the case of pain in the limbs, it will include adaptation of the gait. Recently, first attempts to measure gait adaptations to pain have been developed. Clarke and colleagues (1997) used an optical methodology which allowed gait analysis [19]. Rats were allowed to ambulate spontaneously on a glass floor $(120 \times 12 \times 12 \mathrm{~cm})$ internally illuminated by fluorescent tubes until it had traversed the chamber at least 6 times, or until the expiry of $10 \mathrm{~min}$, whichever was the shorter period. Using a model of ostheoarthrosis, they observed a reduction in the pressure of the affected limb, with redistribution of the loadings to the other limbs. Any disturbance in stride, stance or swing time could be observed. The set-up used by Clarke et al. (1997) results however in irregular speed performance of the rats. But changes in the velocity are known to affect several parameters of the gait [20] and consequently make any comparison of those perilous. Moreover, the analyses of the data are performed through the use of different software's and are therefore time-consuming. Min et al. (2001) have constructed a device to measure the weight load on each leg while the animal is walking [7]. They could observe a significant reduction of weight load on the affected leg after an injection of $1 \mathrm{mg}$ of CAR into the rat knee. The load decreased to the minimum level at 4 hours after injection and gradually returned to the pre-injection level by the fifth day. Coulthard and co-workers (2003) used video recordings from spontaneously walking rats in order to measure gait changes as an objective marker of persistent pain [8]. A bilateral rear intraplantar injection of $6 \mathrm{mg}$ of CAR resulted in temporal and spatial gait changes over a 90 minutes period. The changes consisted 
of a reduction of velocity and stride length as well as an increase in dual stance duration (duration of ground contact for both hind paws simultaneously). However, again, the changes observed by Coulthard et al. (2003) in velocity affect several parameters of the gait and make any conclusion hazardous. In all cases, the techniques used allow the measurement of limited numbers of parameters or with a high work load, strictly related to single paws and no correction for the speed is included.

A recently developed method to analyze gait in rats or mice is the CatWalk [9, 14]. This method has been originally developed a few years ago to assess locomotor deficits in spinal cord injury models [14]. This automated gait analysis method allows an objective quantification of numerous gait related parameters, through the visualization of the foot-floor contact of walking rats in time. Hence, compared with previously described techniques, the CatWalk offers a powerfull tool to assess more than 15 gait related parameters in a single, fast (about $1 \mathrm{~s}$ ) and fully computerized test. Therfore, we hypothesized that the CatWalk method could be an appropriate method to fully and objectively assess gait adaptations in a model of CAR induced inflammatory pain. An important pre-requisite in our experimental set-up was the evaluation of CatWalk runs under constant crossing speed as reported before [20]. The CatWalk parameters were classified into two groups: parameters related to single paws and parameters related to interlimb coordination.

The CatWalk parameters related to single paws are the intensity of the paw print, the duty factor (stance duration/[stance +swing duration]), the base-of-support and the relative paw placement. The signal intensity during placement of the paw during crossing of the CatWalk walkway is an estimate of paw pressure, since signal intensity decreases when less pressure is applied with the paw [21]. The decrease we observe in the affected paw pressure starts being significantly different from the preoperative value at 24 hours after the injection and lasts at least for 48 hours post-injection. This decrease is completely in line with the study of Min et al. [7]. The study of the duty factor of the two pelvic girdle paws reveals a decrease in the paw-floor contact (stance duration) of the ipsilateral paw, associated with an increase in the swing duration (results not shown). An increase in the duty factor of the contralateral paw at the same time reveals a longer paw-floor contact and suggests a compensation behavior for the animal's general weight bearing. As partially discussed by Coulthard et al. (2003), changes in the paw statistics can be due to the avoidance of the non-noxious mechanical stimulation of walking and therefore can be considered as a potential objective marker of pain [8]. The base-of-support of the hind limbs (BOS HL) is a gait parameter that shows how wide the rat walks (the distance between the 2 hind paws). It increases up to $50 \%$ in spinal cord injury in rats [13]. However, our results demonstrate that the CAR-induced knee inflammation does not affect the BOS HL. One can easily hypothesize that change in the distance between the two rear paws 
will not ease the pain in any way. Considering the relative paw placement, it is known that, for their safety, rats are used to place their hind paws on the floor on a position as close as possible from where the fore paws were placed [14]. In this experiment, we show that the inflammatory pain induces a significant shift in the relative placement of the ipsilateral fore and hind paws. We observe a shift backward in the position of the affected hind paw as compared to the position of the ipsilateral front paw, following the CAR injection. These findings reflect temporal (a shorter paw-floor contact) as well as spatial (decrease in the paw pressure; shift in the ipsilateral paw position) changes in gait, after a CAR-induced knee inflammation.

A detailed study of the interlimb coordination can add valuable information, in telling us how precise the rats adapt their gait to the pain. The CatWalk parameters related to the interlimb coordination are the regularity index as well as the phase lag and its variability. First, the regularity index, representing the percentage of normal step sequence patterns of the rat, is a well-known way to measure the degree of coordination. Our results showed no difference in the degree of coordination, after the CAR injection. It reveals a constant percentage of normal step sequence patterns, before vs after CAR injection. Secondly, a detailed analysis of coordination was based on the phase lag between the two pelvic limbs and its variablity. The phase lag relies the initial contact of one paw (the Target) to the stride cycle of another paw (the Anchor) and is expressed in percentage. For instance, one paw could be measured as placed on the floor at $30 \%$ of the stepcycle duration from another paw. After a CAR knee injection, we observed a delay in the use of the affected paw as well as a premature use of the contralateral hind paw. These observations are in line with the reduction of the stance phase duration of the affected paw we described above. All together, this data show a shorter, lighter and delayed paw-floor contact of the ipsilateral paw, following the induction of a CAR-induced inflammation. More interestingly, the variability in the phase lag does not show any difference between the CAR and the control groups nor between the pre- and post-injection values in this study. This suggests a very precise adaptation in the changes in interlimb time relationship. We therefore conclude that the accuracy in the locomotor behavior of the rat is not affected in the CAR induced acute inflammatory pain model.

\section{Development of mechanical allodynia}

The development of a mechanical allodynia can be tested with the use of von Frey filaments [2]. The animals in our study received $2 \mathrm{mg}$ of CAR into the right knee. As a result of this, they developed mechanical allodynia that became significant at $2.5 \mathrm{~h}$ after injection, and then further was maximal at $4 \mathrm{~h}$ when a plateau-like phase was reached. This plateau lasted at least until the last time point investigated, i.e. $48 \mathrm{~h}$. These observa- 
tions are in line with those of Radhakrishnan and colleagues (2003) who also showed a maximum decrease in withdrawal threshold at $4 \mathrm{~h}$ followed by a plateau until at least $24 \mathrm{~h}$ after injecting the same concentration of CAR as used in our study [17].

A correlation between the development of a mechanical allodynia as tested with the von Frey and three of the CatWalk parameters (intensity of the paw print, durations of the stance and swing phases) has already been shown in a chronic pain model in rats, the CCI (chronic construction injury) [4]. Interestingly, our results show that von Frey results are highly correlated to the assessment of single paw related CatWalk parameters. Not only the intensity and the duty factor are strongly correlated (as in Vrinten's paper) in this model of acute pain but also the swing speed, max. area, print area, box width and box length.

Despite the striking effects we observed with both the von Frey and CatWalk techniques, two limitations still need to be discussed. First, it has been shown that food restriction affects pain behavior in rats [22]. In this paper, de los Santos-Arteaga and colleagues fed mice with an alternate-day feeding regimen for 3 months. At the end of this period, the weight of the mice was not affected but the animals displayed markedly reduced responses in models of thermal and visceral pain compared with mice fed ad libitum. The diet presented in their study did not lead to any changes in the rat's weight. In our experiment, food restriction was needed for correct CatWalk measurements. Therefore, rats were fed for 2 weeks with $12-15 \mathrm{~g}$ per day. First of all, the difference in time between the two studies has to be mentioned (12 weeks of diet vs 2 weeks) and does not allowed any direct comparison. Furthermore, even despite this limitation, the CAR group still shows a significant decrease in the withdrawal threshold after the CAR injection, as compared to the control group. Both groups being fed with the same diet, the changes in the sensitivity to pain can only be attrib-

Table I: Pearson Coefficient (p.c.c), as a measure of the correlation between the von Frey results of the right hind paw and the CatWalk parameters related to the right hind paw.

\begin{tabular}{cc} 
von Frey vs... & p.c.c. \\
\hline intensity & 0.94 \\
duty factor & 0.87 \\
swing speed & 0.87 \\
max. area & 0.83 \\
print area & 0.88 \\
box width & 0.93 \\
box length & 0.91
\end{tabular}


uted to the induced inflammatory pain. Second, one could argue that the injection of an inflammatory agent into the knee joint induces a swelling that may affect gait in itself, without being a sign of pain. However, Min et al. [7] showed that the use of morphine $(5 \mathrm{mg} / \mathrm{kg})$ significantly improves the weight load on the arthritic leg in the rat. Although the incomplete recovery of the changes in weight load on the affected paw may be due to mechanical disturbance induced by the edema, Min and colleagues (2001) concluded that weight load measured during walking can be used as a reliable index for the severity of pain. This conclusion together with the high coefficient of correlation we obtained with the von Frey results lead us to conclude that changes observed based on these CatWalk-parameters are mainly due to the development of mechanical allodynia in the affected limb.

In conclusion, the CatWalk analysis of the CAR-induced inflammation model describes an accurately coordinated gait adaptation of the rat to inflammatory pain. It also allows an objective assessment of the development of mechanical allodynia.

\section{Acknowledgment}

This work was funded by a grant from the Dutch Government, NWO-SENTER (to M.A.E.M. and E.A.J.J.). 


\section{References}

1. Le Bars, D., M. Gozariu, and S.W. Cadden, Animal models of nociception. Pharmacol Rev, 2001. 53(4): p. 597-652.

2. Chaplan, S.R., et al., Quantitative assessment of tactile allodynia in the rat paw. J Neurosci Methods, 1994. 53(1): p. 55-63.

3. Dixon, W.J., Efficient analysis of experimental observations. Annu Rev Pharmacol Toxicol, 1980. 20: p. 441-62.

4. Vrinten, D.H. and F.F. Hamers, 'CatWalk' automated quantitative gait analysis as a novel method to assess mechanical allodynia in the rat; a comparison with von Frey testing. Pain, 2003. 102(1-2): p. 203-9.

5. Koopmans, G.C., et al., Circulating insulin-like growth factor I and functional recovery from spinal cord injury under enriched housing conditions. Eur J Neurosci, 2006. 23(4): p. 1035-46.

6. Bech, P., Health-related quality of life measurements in the assessment of pain clinic results. Acta Anaesthesiol Scand, 1999. 43(9): p. 893-6.

7. Min, S.S., et al., A novel method for convenient assessment of arthritic pain in voluntarily walking rats. Neurosci Lett, 2001. 308(2): p. 95-8.

8. Coulthard, P., S.U. Simjee, and B.J. Pleuvry, Gait analysis as a correlate of pain induced by carrageenan intraplantar injection. J Neurosci Methods, 2003. 128(1-2): p. 95-102.

9. Hamers, F.P., et al., Automated quantitative gait analysis during overground locomotion in the rat: its application to spinal cord contusion and transection injuries. J Neurotrauma, 2001. 18(2): p. 187-201.

10. Lankhorst, A.J., et al., Effects of enriched housing on functional recovery after spinal cord contusive injury in the adult rat. J Neurotrauma, 2001. 18(2): p. 203-15.

11. Koopmans, G.C., et al., The assessment of locomotor function in spinal cord injured rats: the importance of objective analysis of coordination. J Neurotrauma, 2005. 22(2): p. 214-25.

12. Gorska, T., W. Zmyslowski, and H. Majczynski, Overground locomotion in intact rats: interlimb coordination, support patterns and support phases duration. Acta Neurobiol Exp (Wars), 1999. 59(2): p. 131-44.

13. Joosten, E.A., W.B. Veldhuis, and F.P. Hamers, Collagen containing neonatal astrocytes stimulates regrowth of injured fibers and promotes modest locomotor recovery after spinal cord injury. J Neurosci Res, 2004. 77(1): p. 127-42.

14. Hamers, F.P., G.C. Koopmans, and E.A. Joosten, CatWalk-Assisted Gait Analysis in the Assessment of Spinal Cord Injury. J Neurotrauma, 2006. 23(3-4): p. 537-48.

15. Schaible, H.G. and B.D. Grubb, Afferent and spinal mechanisms of joint pain. Pain, 1993. 55(1): p. 5-54.

16. Winter, C.A., E.A. Risley, and G.W. Nuss, Carrageenin-induced edema in hind paw of the rat as an assay for antiiflammatory drugs. Proc Soc Exp Biol Med, 1962. 111: p. 544-7.

17. Radhakrishnan, R., S.A. Moore, and K.A. Sluka, Unilateral carrageenan injection into muscle or joint induces chronic bilateral hyperalgesia in rats. Pain, 2003. 104(3): p. 567-77.

18. Lam, F.Y. and W.R. Ferrell, Inhibition of carrageenan induced inflammation in the rat knee joint by substance P antagonist. Ann Rheum Dis, 1989. 48(11): p. 928-32.

19. Clarke, K.A., et al., Gait analysis in a rat model of osteoarthrosis. Physiol Behav, 1997. 62(5): p. 951-4. 
20. Gorska, T., H. Majczynski, and W. Zmyslowski, Overground locomotion in intact rats: contact electrode recording. Acta Neurobiol Exp (Wars), 1998. 58(3): p. 227-37.

21. Betts, R.P. and T. Duckworth, A device for measuring plantar pressures under the sole of the foot. Engineering in Medicine, 1978. 7(4): p. 223.

22. de los Santos-Arteaga, M., et al., Analgesia induced by dietary restriction is mediated by the kappa-opioid system. J Neurosci, 2003. 23(35): p. 11120-6. 



\title{
The CatWalk method: assessment
} of mechanical allodynia in experimental chronic pain?

\author{
A. F. Gabriel'1,3, M. A.E. Marcus', \\ G. H.I.M. Walenkamp², E. A.J. Joosten ${ }^{1,3}$
}

'Department of Anesthesiology, Academic Hospital Maastricht, 6202 AZ Maastricht, the Netherlands

2Department of Surgery and Orthopedics, Academic Hospital Maastricht, 6202 AZ Maastricht, the Netherlands ${ }^{3}$ European Graduate School of Neuroscience (EURON), University of Maastricht, P.O. Box 616,6202 AZ, Maastricht, The Netherlands

Behavioural Brain Research, 198 (2009), 477-480 


\section{Abstract}

The CatWalk gait analysis system has recently been suggested as a rapid and objective alternative method over the von Frey test to assess mechanical allodynia in chronic neuropathic pain models. Our results demonstrate that no correlation exists between the development of mechanical allodynia and changes in CatWalk gait parameters in a chronic inflammatory pain model. Hence, the use of the CatWalk in assessment of experimental chronic pain is discussed. 


\section{Introduction}

The CatWalk technique is a computer-assisted method of locomotor analysis that allows a rapid quantification of individual paw parameters as well as parameters related to interlimb coordination [1]. In the context of experimental pain research, various attempts have been undertaken to analyze gait changes [2-4]. Interestingly, the gait parameters swing phase, stance phase and paw intensity have been suggested as a novel rapid method which may serve as an additional tool in the investigation of mechanical allodynia [5]. In this study it was shown that after a chronic constriction injury (CCI) of the sciatic nerve the adult rat minimizes contact parameters with the affected paw during locomotion, as demonstrated by a reduction in stance phase and in pressure applied stance. These parameters showed a high degree of correlation with mechanical thresholds as determined by the von Frey test. Recently, the CatWalk has been described as a powerful tool to assess pain-induced gait changes in a model of acute inflammatory pain [6]. Also in this study, a high correlation was reported between locomotion related parameters duty factor ( = ratio stance phase/stepcycle) and intensity of the paw print and the von Frey test data.

The claim that the CatWalk gait analysis can be used for objective, rapid and reproducible assessment of mechanical allodynia in rodents [5] has let to the implementation of the commercially available CatWalk system (Noldus Information Technology, Wageningen) in many pain laboratories.

However the question remains if the gait changes observed during locomotion in an experimental pain model do actually reflect the development of mechanical allodynia in the injured paw. In the present investigation, we injected carrageenan (CAR) into the right knee of an adult rat, leading to the development of a knee inflammation. This model is described as a model of acute inflammatory pain during the first $24 \mathrm{~h}$ which later develops into a model of chronic pain after one to two weeks that lasts up to 3 to 8 weeks, depending on the amount of CAR injected [7]. We then investigated whether the CatWalk as compared to the von Frey method does allow detection of mechanical allodynia in the chronic phase of the CAR-induced inflammatory pain model.

\section{Material and methods}

A total of 24 males Sprague-Dawley rats weighing 250-300 g were used and housed individually under standard conditions with a $12 \mathrm{~h}$ light/12 h dark cycle. Both experimental and control rats were trained for two weeks before the onset of the experiment with the CatWalk technique. This training resulted in a cross- 
ing of the pathway without hesitation at a constant speed. Water was available ad libitum. In this study, principles of animal care were followed and the protocol was approved by the Ethics Committee on Animal Experiments of the University of Maastricht (DEC protocol no. 2006/032).

Prior to CAR injection ( $\mathrm{t}=-1$ day), baseline values were obtained for CatWalk and von Frey tests. The experimental group $(n=16)$ was injected intra-articularly, at $t=0$, with $2 \mathrm{mg}$ of $\lambda$-Carrageenan (in $200 \mu \mathrm{l}$ of saline solution) (Sigma-Aldrich, no.C3889) into the rat right knee. Control animals $(n=8)$ were injected identically with a saline solution $(200 \mu \mathrm{l})$. CatWalk and von Frey tests were performed one day after the injection and from then on, once a week, up to 4 weeks.

Mechanical allodynia was assessed using the von Frey test by measuring paw withdrawal threshold in response to mechanical stimuli. Animals were placed in a plastic cage with a metal mesh floor, allowing them to move freely. Filaments of various thicknesses (North Coast Medical, Inc., CA, USA) were applied to the midplantar surface of both rat hind paws. A minimum of three withdrawals of the tested paw out of five filament applications was considered as a positive response. Filaments were applied in ascending order, and the smallest filament that elicits a positive response was considered the threshold stimulus.

The CatWalk method is a detailed analysis of gait performed on walking rats [8], [9], [6]. Briefly, light from a fluorescent tube is sent through a glass plate. Light rays are completely reflected internally. As soon as the rat's paw is in contact with the glass surface, light is reflected downwards. It results in a sharp image of a bright paw print. The whole run is recorded by a camera. Sweet pellets (Sandown Chemical Ltd., UK) are placed at the end of the walkway. Animals are motivated via a food restriction protocol (12-15 $\mathrm{g}$ of food is given at the same time every day to the rat, right after that the behavioral test is performed). In the present study, general parameters were collected (duration of the run). Parameters related to single paws were also analyzed (Intensity of the paw print; Duty factor -i.e. ratio between stance duration and stepcycle duration-). The intensity of the paw print is expressed in arbitrary units (a.u.). This parameter reflects the mean pressure exerted by one individual paw during the floor contact, during the whole crossing of the walkway. Data analysis was performed with a threshold value of 40 (arbitrary units, possible range 0-225), i.e. all pixels brighter than 40 are used. The Duty factor is expressed in \%. It represents the ratio between the stance duration and the stepcycle duration. It is calculated according to the formula: stance phase duration/ [stance + swing phases duration] $\times 100$ [10], where the stance duration is the time of contact of one paw with the floor during one single stepcycle and the swing duration is the time of non-contact with the floor during the stepcycle. The time 
contact (or stance phase duration) of one paw can be considered as directly related to the perceived pain in the limb, as the duty factor therefore is. According to its definition, this parameter cannot be defined if the paw is not placed on the floor.

In this study, CatWalk parameters (duty factor and intensity of the paw print) and von Frey withdrawal threshold were calculated as a percentage of respective pre-operative values, identical to the data-presentation in Vrinten and Hamers's article [5]. As such, each animal acts as its own control. All data are expressed as mean \pm S.E.M. To compare difference over time of each parameter, analysis of variances (ANOVA's) were performed, followed by paired t-tests (comparison with pre-operative values). The Pearson correlation coefficient ( $r$ ) was used to study a possible correlation between the von Frey data and CatWalk parameters related to a single paw.

\section{Results}

Paw withdrawal threshold to mechanical stimulation was assessed using the von Frey test. The intra-articular injection of CAR produced a significant decrease in the withdrawal threshold to $10.6 \pm 9.2 \%$ of pre-operative value at Day Post-Operation 1 (DPO1) and up to $32.1 \pm 13.3 \%$ at DPO21 $(\mathrm{p}<0.05)$ (Figure 1$)$. The observed increased sensitivity remained exclusively ipsilateral. At DPO28, the withdrawal threshold did no longer significantly differ from pre-operative baseline values. No effect was observed in control animals over time (results not shown).

Detailed analysis of gait was performed on walking rats using the CatWalk method. In this study, locomotor speed ranged between $60-80 \mathrm{~cm} / \mathrm{s}$ for the whole experiment and did not differ between the experimental and the control groups.

Individual paw parameters studied are the intensity of the paw print and the duty factor of the ipsilateral paw. CAR injection resulted in a temporary significant decrease of the intensity of the ipsilateral paw print to $81.6 \pm 2.9 \%$ of pre-operative value at DPO1 $(\mathrm{p}<0.05)$ (Figure 1). From DPO7 on, the paw print intensity of the injured hindlimb was no longer statistically decreased compared to pre-operative value. Besides, the influence of the rat weight on the paw intensity was assessed. No significant difference could be noted between the paw intensity of rats weighing $230 \mathrm{~g}$ versus $250 \mathrm{~g}$. (i.e. a variation of about $10 \%$ in weight) $(\mathrm{p}=0.87$ ). Rats weighing $330 \mathrm{~g}$ showed a significantly different intensity of the paw print than rats weighing $230 \mathrm{~g}$ (i.e. a variation of about $40 \%$ in weight) $(\mathrm{p}=0.03$ ). No difference in paw print intensity was noted between rats weighing 330 and $250 \mathrm{~g}$ (i.e. a variation of $32 \%$ in weight $)(p=0.12)$. 


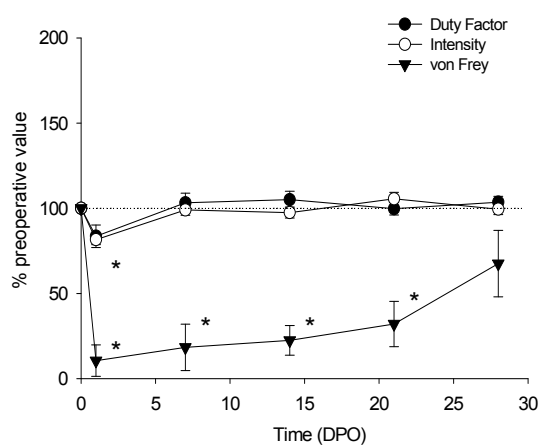

Figure 1: Time course of CatWalk parameters (Intensity and Duty factor) and von Frey withdrawal threshold in a rat model of inflammatory pain. Measurements were taken pre-operatively and at DPO (Day Post-Operation) $1,7,14,21$ and 28. Values are expressed as percentage of respective pre-operative values. Data are expressed as mean \pm s.e.m. The symbol $\left(^{*}\right)$ indicates values significantly different from baseline $(p<0.05)$.

Finally, CAR injection provoked a significant decrease in the duty factor of the ipsilateral paw to $83.6 \pm 6.6 \%$ of pre-operative value at DPO1 $(\mathrm{p}<0.05)$ (Figure 1 ). From $\mathrm{DPO} 7 \mathrm{on}$, the duty factor did no longer significantly differ from preoperative value.

No significant correlation could be noted between the von Frey results and CatWalk parameters $(\mathrm{r}$ [CatWalk intensity and von Frey] $=0.51 ; \mathrm{r}$ [CatWalk duty factor and von Frey] $=0.48)$.

\section{Discussion}

In this study, we demonstrated the absence of any correlation between mechanical withdrawal threshold, as assessed with the von Frey test, and the CatWalk parameters, Intensity and Duty factor, during the chronic phase of an inflammatory pain model. The discrepancy between our results and those reported in previous studies $[5,6]$ will be discussed.

Changes in the mechanical sensitivity of the paw can be assessed using the von Frey test, a golden standard algesimetry test [11]. Our results demonstrated the development of a mechanical allodynia in the ispsilateral paw of the rat at DPO1 following an injection of CAR $(2 \mathrm{mg})$ into the right knee (Figure 1). These findings are identical to those reported earlier [6]. In the chronic phase, the CAR-induced mechanical allodynia remained exclusively ipsilateral and significant up to DPO21. At DPO28, no increased sensitivity of the ipsilateral limb could be observed anymore. These results are in line with and can be extrapolated from the study of Radhakrishnan et al. [7]. 
Beside the direct assessment of response-to-stimulus tests, like the von Frey test, several attempts have been undertaken to evaluate behavioral adaptations in experimental pain models, as an indirect measure of pain. Among these is the assessment of gait changes. Recently, the CatWalk technique has been demonstrated as one of the most complete and objective methods of gait analysis $[8,12]$. Therefore, in this study, we used the CatWalk technique to assess the long term effect of a CAR injection into the right knee of the rat on pain-induced gait changes. The most important finding in this respect is the fact that CatWalk parameters (Intensity and Duty factor) do no longer differ from pre-operative levels during the chronic stages of the CAR-induced inflammatory pain although the difference was significant in the 48 first hours postinjection (acute phase). Hence, a full recovery of the CAR-induced gait changes was described already at one week after the knee injection, whereas at the same time, the mechanical allodynia, as assessed by the von Frey test, was significant during both acute and chronic stages of this model (up to four weeks post-injection). Interestingly, long lasting gait changes have been described in other experimental pain models, as for instance the chronic constriction injury (CCI) model in the rat [5] or after a complete sciatic nerve transection [13]. In these studies, CatWalk-gait parameters (paw print intensity, stance and swing phases) showed significant changes which concur with the mechanical allodynia assessed with the von Frey test in the chronic phase of the model. In both models, the sciatic nerve was directly injured and this resulted in long lasting gait changes. From this, we can conclude that the use of the CatWalk based gait analysis as a pain test as well as the correlation of its data with the development of mechanical allodynia is highly dependent on the pain model used. Here the presence of nerve damage may be pivotal.

Beside this, the difference between von Frey-mechanical allodynia assessment and CatWalk-based gait changes can largely be explained by major differences between the biological mechanisms underlying the response assessed in these two behavioral tests. It is known that nociceptive cutaneous afferents are triggered following a mechanical noxious stimulus. In the von Frey test, the rat will withdraw its paw away from the noxious mechanical stimulus of the von Frey filament. The signal goes from the skin's paw to the spine and back to the muscles. After a few milliseconds, the brain receives the noxious stimulus and translates it as a pain sensation. Supraspinal controls are not considered to play a major role in this spinally-mediated response. On the other hand, gait in mammals is controlled by networks of spinal neurons together forming so-called central pattern generators (CPG). Descending pathways, mainly originating in the brainstem, are involved in the initiation, maintenance, coordination and constant adaptation of CPG-mediated rhythmic movements [14-16]. Furthermore, these brainstem nuclei are under the control of higher brain areas as the sensorimotor cortex, the cerebellum and the basal ganglia [15]. Therefore, nociceptive input and the subsequent pain sensation are expected to influ- 
ence the CPG-controlled gait pattern. Indeed, numerous studies have demonstrated long-term adaptation of a rhythmic behavior following a sudden change in afferent signals [16]. These adaptations can occur over periods of hours or days. All together, these data demonstrate that behavioral adaptation to pain is cortically-mediated and initiated by the conscious sensation of pain asking for attention. However, it has been demonstrated that the attentional cost of such an adaptative behavior is highly dependent on the degree of exposure to pain [17]. Habituation to pain stimulus is thought to reduce the threat value of the pain signal and its subsequent adaptative behavior [18]. Locomotion can be considered like a daily life activity, i.e. it is a voluntarily initiated movement, repeated several times a day. Therefore the phenomenon of habituation is very likely to take place. This is in line with our findings: during the first $24 \mathrm{~h}$ (DPO1) after the CAR injection significant gait changes are noted whereas after one week (DPO7), no gait changes are detected anymore by the CatWalk, although the oversensitivity as expressed by the development of mechanical allodynia is still present according to the von Frey data.

Finally, the discrepancy in timing between the development of mechanical allodynia and CatWalk based pain-induced gait changes in this model raises once again the question of the pertinence of translation of the outcomes from such experimental behavioral tests to the clinic (for instance when using these tests for drug preclinical screening). Although the von Frey test has been used worldwide as a golden standard, we must keep in mind that it only gives us a narrow view of the whole pain picture. It is a strict measure of nociception (i.e. neuronal activity triggered by a noxious stimulus) whereas gait is a higher centers-controlled process and its analysis through the CatWalk technique might be closer to the human chronic pain syndromes (e.g. interferences with daily life activities, unpleasantness of pain) and hence, clinically more relevant. Besides, the application of the von Frey filament before the paw withdrawal on one narrow area of the rat plantar surface for one or two seconds is likely to be a stronger stimulus than the touch of the paw with the floor when walking during the CatWalk test and bearing alternatively the weight on every paw during few milliseconds. Indeed, the pressure applied on each individual mechanoreceptor as well as the amount of skin deformation during the von Frey test is likely to be higher than during locomotion on the CatWalk. In that sense, von Frey results are likely overstating the model as compared to the assessment of a voluntary daily life activity like walking. Here again, analysis of gait changes as assessed with the CatWalk turns out to be clinically more relevant and closer to clinical chronic pain syndromes.

Nevertheless, it seems to the authors that a last note on the sensitivity of the CatWalk parameter paw Intensity is worth mentioning. Indeed, it should be taken into account that the intensity of the rat paw print, reflecting the pressure applied on the paw, is rather limited in sensitivity. As observed in our results, a variation of 10 
to $30 \%$ in weight does not significantly influence the CatWalk parameter Intensity. A variation of at least $40 \%$ in weight is required for a significant difference to be observed in the intensity of the paw print. Therefore, CAR-induced changes in the intensity of the paw print as observed in this study are reflecting a difference in paw pressure equivalent to a change of at least $40 \%$ of weight applied on that paw.

All together, we first conclude that the previously claimed use of the CatWalk as a method to assess pain-induced gait changes in correlation with mechanical allodynia $[5,6]$ needs to be carefully restricted to identified experimental pain models.

Second, our results also indicate that a simultaneous use of both von Frey and CatWalk tests to assess pain in experimental pain research will result in a better and more reliable picture of the whole pain experience in the rat.

\section{Acknowledgment}

This study was funded by a grant from the Dutch Government, NWO-Senter (to M.A.E.M. and E.A.J.J.) and it was performed within TREND (Trauma Related Neuronal Dysfunction), a knowledge consortium that integrates research on CRPS type I, and is supported by a Dutch government grant (BSIK 03016). The author thanks Dr R. Deumens for critical reading of the manuscript. 


\section{References}

1. Koopmans, G.C., et al., Circulating insulin-like growth factor I and functional recovery from spinal cord injury under enriched housing conditions. Eur J Neurosci, 2006. 23(4): p. 1035-46

2. Min, S.S., et al., A novel method for convenient assessment of arthritic pain in voluntarily walking rats. Neurosci Lett, 2001. 308(2): p. 95-8

3. Coulthard, P., S.U. Simjee, and B.J. Pleuvry, Gait analysis as a correlate of pain induced by carrageenan intraplantar injection. J Neurosci Methods, 2003. 128(1-2): p. 95-102

4. Clarke, K.A., et al., Gait analysis in a rat model of osteoarthrosis. Physiol Behav, 1997. 62(5): p. 951-4

5. Vrinten, D.H. and F.F. Hamers, 'CatWalk' automated quantitative gait analysis as a novel method to assess mechanical allodynia in the rat; a comparison with von Frey testing. Pain, 2003. 102(1-2): p. 203-9

6. Gabriel, A.F., et al., The CatWalk method: a detailed analysis of behavioral changes after acute inflammatory pain in the rat. J Neurosci Methods, 2007. 163(1): p. 9-16

7. Radhakrishnan, R., S.A. Moore, and K.A. Sluka, Unilateral carrageenan injection into muscle or joint induces chronic bilateral hyperalgesia in rats. Pain, 2003. 104(3): p. 567-77

8. Hamers, F.P., et al., Automated quantitative gait analysis during overground locomotion in the rat: its application to spinal cord contusion and transection injuries. J Neurotrauma, 2001. 18(2): p. 187-201

9. Lankhorst, A.J., et al., Effects of enriched housing on functional recovery after spinal cord contusive injury in the adult rat. J Neurotrauma, 2001. 18(2): p. 203-15

10. Gorska, T., W. Zmyslowski, and H. Majczynski, Overground locomotion in intact rats: interlimb coordination, support patterns and support phases duration. Acta Neurobiol Exp (Wars), 1999. 59(2): p. 131-44

11. Chaplan, S.R., et al., Quantitative assessment of tactile allodynia in the rat paw. J Neurosci Methods, 1994. 53(1): p. 55-63

12. Koopmans, G.C., et al., The assessment of locomotor function in spinal cord injured rats: the importance of objective analysis of coordination. J Neurotrauma, 2005. 22(2): p. 214-25

13. Deumens, R., et al., The CatWalk gait analysis in assessment of both dynamic and static gait changes after adult rat sciatic nerve resection. J Neurosci Methods, 2007. 164(1): p. 120-30

14. Jordan, L., et al., Descending command systems for the initiation of locomotion in mammals. Brain Res Rev., 2008. 57(1): p. 183-91

15. MacKay-Lyons, M., Central pattern generation of locomotion: a review of the evidence. Phys Ther, 2002. 82(1): p. 69-83

16. Pearson, K.G., Neural adaptation in the generation of rhythmic behavior. Annu Rev Physiol, 2000. 62: p. $723-53$

17. Crombez, G., et al., Habituation and the interference of pain with task performance. Pain, 1997. 70(2-3): p. 149-54

18. Moseley, G.L. and P.W. Hodges, Are the changes in postural control associated with low back pain caused by pain interference? Clin J Pain, 2005. 21(4): p. 323-9 


\title{
The CatWalk method: a sensitive and objective tool for preclinical
}

\section{assessment of analgesic drugs}

\author{
A. F. Gabriel',2, W. M.M. Honig', M. A.E. Marcus', E. A.J.Joosten ${ }^{1,2}$
}

'Department of Anesthesiology, Academic Hospital Maastricht, 6202 AZ Maastricht, the Netherlands

'European Graduate School of Neuroscience (EURON), University of Maastricht, P.O. Box 616, 6202 AZ, Maastricht, The Netherlands

Submitted 


\section{Abstract}

For the assessment of analgesic drug effects in acute experimental postoperative pain models, the von Frey test is considered to be a golden standard for the detection of effects on mechanical allodynia. The CatWalk automated quantitative gait analysis method has been shown to allow objective measurement of gait changes which are highly correlated to the development of mechanical allodynia as assessed by the von Frey test. However, up till now the ability and potency of the CatWalk-system to detect analgesic drug effects in animal models for acute post-operative pain has never been demonstrated. Therefore the present investigation was aimed at the analysis of the analgesic effect of the drug Fentanyl on various CatWalk parameters in an acute inflammatory pain model. Two mg of Lambda-carrageenan was intra-articularly injected into the right knee of adult rats. Twenty-four hours later $25 \mu \mathrm{g} / \mathrm{kg}$ Fentanyl was injected subcutaneously. Then CatWalk and von Frey analyses were performed preoperatively, prior to the Fentanyl injection and at 20,45 and 120 min post-injection. Our results demonstrate a significant effect of Fentanyl not only on mechanical allodynia as assessed with the von Frey method but also a significant effect on various CatWalk-parameters. It clearly comes out that the CatWalk gait analysis results in a more objective, more sensitive and more reproducible testing of the analgesic effects of Fentanyl in this experimental model as compared to the von Frey technique. We therefore highly recommend the CatWalk gait analysis for testing of analgesic drugs in experimental models for acute postoperative pain. 


\section{Introduction}

The CatWalk technique is an automated gait analysis system for rodents. It has been originally developed as a tool to assess functional recovery after spinal cord injury $[1,2]$.

Vrinten et al. were the first ones to introduce the CatWalk technique in the field of experimental pain assessment [3]. In a CCI (Chronic Constriction Injury) rat model, they reported a high correlation between changes in CatWalk-based gait parameters (intensity of the paw print, swing and stance phases) and the development of mechanical allodynia as measured by the von Frey test. In an acute inflammatory pain model (carrageenan(CAR)-injection into the knee), we were also able to demonstrate this correlation (4-48 h post injection) [4]. However, the proof of principle for the causality of mechanical allodynia in the CatWalk-based gait adaptation lies in the ability of the CatWalk-system to detect analgesic drug effects. Analgesic (candidate)-drugs presently are routinely evaluated in experimental pain models and recommended for clinical trials based on their von Frey responses [5, 6]. In this study, we used Fentanyl as a standard analgesic drug. Fentanyl is known to induce a significant increase on the von Frey-withdrawal threshold in numerous pain models and in particular in a CAR-induced inflammatory pain [7].

This study aims to measure the effect of Fentanyl on various CatWalk parameters in an acute inflammatory pain model in rats. Results were compared to the effect of Fentanyl on the von Frey response.

\section{Material and methods}

\section{Animals}

Sprague-Dawley male rats weighing 250-300 g were used. Animals were housed individually under standard conditions with a $12 \mathrm{~h}$ light/12 h dark cycle. Rats were fed once a day at a fixed time (12 to $15 \mathrm{~g}$ of food per animal per day) during the 2 weeks period of training with the CatWalk technique as well as during the experiment. Water was available ad libitum. This study was approved by the Ethics Committee on Animal Experiments of the University of Maastricht (DEC protocol no. 2006/066). 


\section{Design of the experiment}

According to the literature [8], an expected effect of $40 \%$ with a possible variation of $20 \%$ were estimated. From this, the actual number of rats per group was determined at 6 (Sachs formula: $\left.\mathrm{n}=2\left(\mathrm{z}_{\mathrm{a} / 2}-\mathrm{z}_{\pi}\right)^{2} \cdot(\sigma / \delta)^{2}\right)$. Two rats were excluded from the control group due to technical problems.

Prior to carrageenan injection, pre-operative values were obtained for CatWalk and von Frey tests (pre-operative value). All 10 animals were injected intra-articularly, at $t=-1$ day, with $2 \mathrm{mg}$ of $\lambda$-Carrageenan (Sigma-Aldrich, no. C3889) into the knee joint of the right hind limb under isofluorane (2.5\%) anesthesia. Prior to experiment, baseline values were obtained for CatWalk and von Frey tests (preFentanyl value). The experimental group $(n=6)$ was injected subcutaneously with Fentanyl (FEN) $(25 \mu \mathrm{g} / \mathrm{kg}$; Bipharma, no. 605066) at $t=0$. The control group $(\mathrm{n}=4)$ was injected with saline solution. CatWalk and von Frey tests were performed at 20, 45 and 120 minutes post-Fentanyl (or saline)-injection.

\section{Assessment of mechanical allodynia with the von Frey test}

Mechanical allodynia was assessed using the von Frey test by measuring paw withdrawal threshold in response to mechanical stimuli. Animals were placed in a plastic cage with a metal mesh floor, allowing them to move freely. They were placed in this cage $10 \mathrm{~min}$ prior testing to allow them to acclimatize to the environment. Filaments of various thicknesses (North Coast Medical, Inc., CA, USA) were applied to the mid-plantar surface of both rat hind paws. Each probe was applied to the foot until it just bent. Then the regularly calibrated stiffness corresponds to an applied force of $0.16,0.4,0.6,1.0,1.4,2.0,4.0,6.0,8.0,10,15,26,60$ and $100 \mathrm{~g}$. A minimum of three withdrawals of the tested paw out of five filament applications was considered as a positive response. Filaments were applied in ascending order, and the smallest filament that elicits a positive response was considered as the threshold stimulus.

\section{Assessment of pain-induced gait adaptation with the Catwalk method}

The CatWalk method is a detailed analysis of gait performed on walking rats [2, 4, 9]. Briefly, light from a fluorescent tube is sent through a glass plate. Light rays are completely reflected internally. As soon as anything, e.g. a rat's paw, is in contact with the glass surface, light is reflected downwards. It results in a sharp image of a bright paw print. The whole run is recorded by a camera placed under the glass plate. Sweet pellets (Noyes Precision pellets PJPPP-045; Sandown Chemical Ltd., Hampton, UK) are placed at the end of the walkway. Animals are motivated via 12- 
15 g per day food time restriction protocol. In the present study, general parameters were collected (duration of the run) as well as parameters related to single paws (Intensity of the paw print, Stance phase duration, Maximum area, Print area, Box width, Box length). A full and detailed description of these parameters is reported in previous paper [4].

\section{Assessment of the Fentanyl-induced recovery in behavioral tests}

Recovery from pain as assessed by the behavioral tests was measured as a comparison (\%) between the CAR effect and the Fentanyl effect. The CAR effect is the difference between the pre-operative value and the pre-Fentanyl value. The Fentanyl effect is the difference between the pre-Fentanyl value and the maximal effect of Fentanyl (value at 20 minutes post-Fentanyl injection).

\section{Statistical analysis}

The global effect of the Fentanyl injection on CatWalk parameters as well as on von Frey withdrawal threshold was evaluated by means of an ANOVA test (repeated measures; $\mathrm{p}<0.05$ ). For each parameter, specific comparison of every time points with pre-Fentanyl value has been statistically tested via a post-hoc comparison followed by a Bonferroni correction.

All data are presented as a ratio between right and left hindpaw's values. They are expressed as mean \pm S.E.M.

\section{Results}

A preliminary study has been performed in order to determine the optimal dose of Fentanyl for induction of a maximal analgesic effect and at the same time with a minimal sedative effect. During this preliminary study, the analgesic effect of different doses of Fentanyl was controlled through the study of both the von Frey withdrawal threshold and the Catwalk's paw intensity. The presence of a sedative effect confounding the results was excluded by considering exclusively Fentanyl concentrations with which the total duration of the Catwalk run was constant as compared with the preoperative value. Among other, an injection of $37.5 \mu \mathrm{g} / \mathrm{kg}$ led to a major sedative effect and this concentration was therefore not considered to be used. Our pilot experiment furthermore showed the absence of an analgesic effect following an injection of $12.5 \mu \mathrm{g} / \mathrm{kg}$. Hence, the final dose used in our experiment was fixed at $25 \mu \mathrm{g}$ of Fentanyl $/ \mathrm{kg}$. 


\section{Assessment of mechanical allodynia with the von Frey test}

Two out of the six rats from the experimental group were excluded from the von Frey test: in these animals, the CAR injection resulted in a complete contraction of the ipsilateral paw. Therefore, the paw surface was no longer available for any correct application of the von Frey filament.

The mean preoperative withdrawal threshold ratio was $0.73 \pm 0.16$. In all animals (from both the Fen group and the Control group), the intra-articular injection of carrageenan resulted in a significant and exclusively ipsilateral decrease in the von Frey withdrawal thresholds after 24 hours, as previously described $[4,10]$.

The Fentanyl injection produced a significant increase in the ratio between the withdrawal thresholds of both hindpaws $\left(\mathrm{F}_{3,9}=4.680 ; \mathrm{p}<0.05\right)$ at 20 minutes post Fentanyl injection. The value of this ratio showed a recovery of $88 \%$ towards the pre-operative value. At 45 minutes, the effect was not significant anymore. No effect was observed over time following a saline injection (data not shown).

\section{Assessment of pain-induced gait adaptation with the Catwalk method}

\section{Run duration}

In the present study, the total run duration ranged between 1.41 and 1.90 s. Fentanyl injection did not result in significant changes in the duration of the whole run.

\section{Parameters related to single paws}

Individual paws parameters studied are the intensity of the paw print, the maximal paw-floor contact area, the total print area, the width and length of the total area and the stance duration.

The CAR injection resulted after 24 hours in a significant decrease of the ratio between the intensity of the hindlimb prints, i.e. a decrease from $0.99 \pm 0.04$ to $0.24 \pm 0.11$ (Figure $2 \mathrm{a}$ ). Then a significant recovery of $81 \%$ was measured $20 \mathrm{~min}$ utes after the Fentanyl injection $\left(\mathrm{F}_{3,15}=8.108\right.$; $\left.\mathrm{p}<0.05\right)$. The recovery in the ratio between the paw print intensities was still significant at 45 minutes post-injection (PI). At 120 minutes PI, this ratio did no longer statistically differ from the preFentanyl value. No effect was observed following a saline solution in the control group (data not shown).

As indicated in Figure $2 b$, the preoperative value of the ratio between stance durations of the two hindlimbs was $0.96 \pm 0.02$. The CAR injection provoked a significant decrease in the stance duration of the ipsilateral paw after 24 hours. After the Fentanyl 


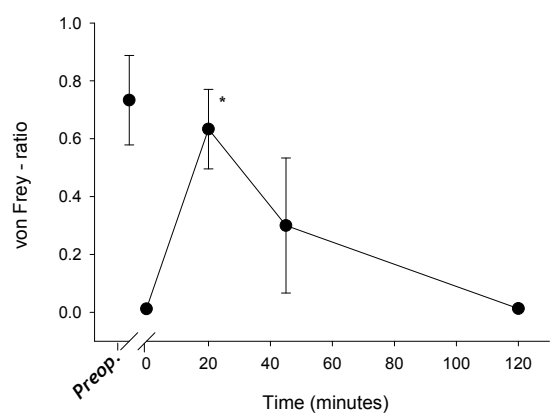

Figure 1: Von Frey analysis: time course of paw withdrawal thresholds following mechanical stimulation. Measurements were taken pre-operatively (preop) and before the Fentanyl injection (pre-Fentanyl; $t=0$ ) and then at 20,45 and 120 minutes post Fentanyl injection. Thresholds are expressed in grams. Values are expressed as ratio of the absolute values of both hindpaws (Right by Left). Data are presented as mean \pm s.e.m. The symbol $\left(^{*}\right)$ indicates data which significantly differ from pre-Fentanyl value $(p<0.05)$.

injection, the value of the stance phase' ratio almost completely recovered towards pre-operative value $(82 \%)\left(\mathrm{F}_{3,15}=6.645 ; \mathrm{p}<0.05\right)$. The effect of Fentanyl on the stance phase duration significantly differed from the pre-Fentanyl value up to 45 minutes. The saline injection did not affect the stance duration over time (results not shown).

The application of Fentanyl also significantly affected other single paw CatWalkrelated parameters like print area, max area, box width, box length. The characteristics of the Fentanyl effect on these CatWalk-parameters are summarized in Table 1.

As illustrated in Table 1, the general effect of Fentanyl injection on behavioral analysis (Table1) was significant in both von Frey and CatWalk tests. However, von Frey analysis resulted in a significant effect of Fentanyl at 20 minutes PI only whereas the Catwalk analysis allowed the detection of significant effects at 20 but also 45 minutes PI. When comparing the $\mathrm{p}$ values, the effects detected with the CatWalk show much lower variability $(\mathrm{p}<0.005)$ than the effect detected by the von Frey test $(\mathrm{p}<0.05)$.

\section{Discussion}

The present study demonstrates that the CatWalk allows a fast, sensitive and objective measurement of the analgesic effect of Fentanyl on gait changes in rats following a CAR-induced knee inflammation. Whereas the CatWalk-based gait changes are related to a CAR-induced mechanical allodynia, we strongly recommend the CatWalk analysis for the assessment of analgesic drug effect in experimental acute pre-operative pain models. 
Several attempts have been undertaken to measure gait changes in experimental pain models [11-14]. Coulthard (2002) as well as Min (2001) demonstrated that gait changes observed in inflammatory pain models could be reversed by the use of opioids $[11,14]$. They concluded that the behavioral changes in gait represented efficient pain parameters. Although the techniques they described resulted in valuable information about pain-induced gait changes, the CatWalk technique is the first one to allow a fast and fully automated measurement of numerous gait parameters. First, Vrinten and Hamers have shown the correlation between CatWalkbased gait changes (intensity of the paw print, stance and swing phase duration) with the von Frey threshold in a rat CCI neuropathic pain model [3]. We confirmed this correlation in a previous study using an acute inflammatory pain model [4]. Moreover, the CatWalk allowed us to fully and objectively describe a fine and tuned pain-induced gait adaptation on walking rats following a carrageenan-induced knee inflammation.

The carrageenan (CAR)-induced inflammation into the knee used in this study has been first described by Winter as acute, non-immune and highly reproducible [15]. The intra-articular injection of $2 \mathrm{mg}$ of CAR in rat knee leads to the development of inflammation-induced allodynia in the ipsilateral limb [4].

Fentanyl was used in this study as a standard analgesic drug. Fentanyl has a strong analgesic potency and an acceptable side-effect profiling compared to other opioids at equianalgesic dose [16]. Among other side-effects, Fentanyl is known to induce a sedative effect [17]. Hence, an important prerequisite to this experiment was the establishment of the appropriate concentration of Fentanyl that may re-
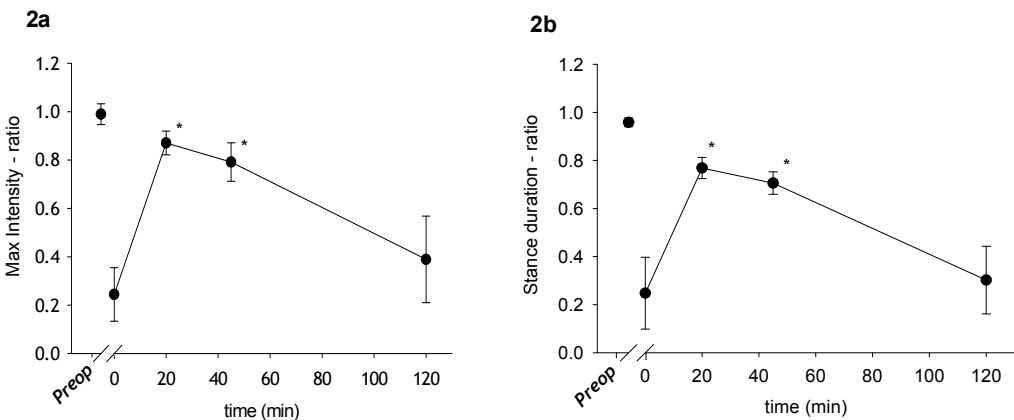

Figure 2: CatWalk analysis: time course of the Intensity of the paw print (2a), the stance phase (2b). Measurements were taken pre-operatively (preop) and before the Fentanyl injection (pre-Fentanyl; $\mathrm{t}=0$ ) and at 20, 45 and 120 minutes post Fentanyl injection. Values are expressed as ratio of absolute values of both hind paws (Right by Left). Data are presented as mean \pm s.e.m. The symbol $\left(^{*}\right)$ indicates data which significantly differ from pre-Fentanyl value $(p<0.05)$. 
Table 1: Effect of Fentanyl on von Frey and CatWalk parameters. The duration of the therapeutic window represents the time points after the Fentanyl injection significantly different from the pre-Fentanyl value. The effect of Fentanyl is the $p$ value of the statistical test. The peak time is the time point where the maximal effect of Fentanyl injection is observed (maximal percentage of recovery). The peak effect is the maximal percentage of recovery measured as percentage of the distance between the pre-operative and the pre-FEN values. $N$ is the number of rats included in the measure.

\begin{tabular}{|c|c|c|c|c|c|c|c|}
\hline & & \multicolumn{2}{|c|}{$\begin{array}{c}\text { Fentanyl Therapeutic } \\
\text { Window }\end{array}$} & \multirow{3}{*}{$\begin{array}{l}\text { Peak time } \\
\text { (minutes post inj) }\end{array}$} & \multirow{3}{*}{$\begin{array}{l}\text { Peak effect } \\
\text { (\% of recovery) }\end{array}$} & \multirow{3}{*}{ Peak s.e.m. } & \multirow{3}{*}{$\underset{\text { (rats }}{\mathbf{n}}$} \\
\hline & & $\begin{array}{l}\text { Duration } \\
\text { (minutes post }\end{array}$ & $\begin{array}{l}\text { Effect } \\
\text { (ANOVA; }\end{array}$ & & & & \\
\hline & & FEN-inj) & p value) & & & & \\
\hline von Frey changes & $\begin{array}{c}\text { Withdrawal } \\
\text { Threshold }\end{array}$ & $0-20 \mathrm{~min}$ & 0.032 & $+20 \mathrm{~min}$ & $88.8 \%$ & 0.14 & 4 \\
\hline \multirow[t]{6}{*}{ CatWalk changes } & Max Area & $0-45 \min$ & 0.002 & $+20 \min$ & $52.2 \%$ & 0.06 & 6 \\
\hline & Max Intensity & $0-45 \mathrm{~min}$ & 0.002 & $+20 \min$ & $80.6 \%$ & 0.05 & 6 \\
\hline & Print Area & $0-45$ min & 0.001 & $+20 \mathrm{~min}$ & $49.9 \%$ & 0.07 & 6 \\
\hline & Box width & $0-45 \min$ & 0.001 & $+20 \mathrm{~min}$ & $55.1 \%$ & 0.05 & 6 \\
\hline & Box length & $0-45 \min$ & 0.002 & $+20 \mathrm{~min}$ & $62.1 \%$ & 0.09 & 6 \\
\hline & Stance duration & $0-45 \mathrm{~min}$ & 0.005 & $+20 \min$ & $81.6 \%$ & 0.04 & 6 \\
\hline
\end{tabular}

sult in a measurable analgesic effect, without any sedative effect. Based on pilotexperiment (unpublished data), the Fentanyl concentration was fixed at $25 \mu \mathrm{g} / \mathrm{kg}$. Using this concentration, behavioral tests could be consistently performed as from 20 minutes post-injection. Moreover, this Fentanyl concentration is described to be in a safe concentrations' range [16]. Meert and Vermeirsch demonstrated that this concentration of Fentanyl exclusively induced an analgesic effect without any side effect, within the time-window of two hours after injection in a formalin-induced inflammatory pain model.

In our experiment, the Fentanyl injection led to a partial recovery (88\%) of the CAR-induced mechanical allodynia, as assessed by the von Frey test. The von Frey test is presently known as a golden standard technique in both clinical and experimental pain assessment to measure analgesic effect [18-20]. Likewise, the subcutaneous injection of Fentanyl also led to a partial recovery (from 50 to 82\%) of CAR-induced gait changes, as assessed by the CatWalk technique. It is the very first time that the causality of pain in CatWalk parameters changes in an experimental pain model is demonstrated. Among all CatWalk-based gait parameters studied, a maximal analgesic effect was observed with two parameters: first, the intensity of the paw print, which is related to the pressure applied on the paw, and second, the stance duration, which is the duration of the paw-floor contact. Our data suggests that the Fentanyl injection allows almost a complete recovery ( 81 and $82 \%$ ) of the ipsilateral paw pressure as well 
as of the duration of paw-floor contact but allows only a partial recovery (from 50 to $62 \%$ ) of the size of the paw-floor contact area (print area, max area, box width and box length) in a model of carrageenan-induced inflammatory pain.

Although our results demonstrate that both the von Frey and the CatWalk tests can be used to assess analgesic effects in a model of acute inflammatory pain, we would like to stress various advantages in the use of the CatWalk gait analysis. The first one is the sensitivity of the technique. The length of the therapeutic window (i.e. time points after the Fentanyl injection significantly different from the pre-Fentanyl value) is longer with the CatWalk than with the von Frey test. The withdrawal threshold, as assessed by the von Frey test, only significantly differed from the preFentanyl value at 20 minutes after Fentanyl injection. With the CatWalk technique, significant changes were detected in all individual paw-related parameters included in this study up to 45 minutes after Fentanyl injection. In other words, the Fentanyl effect can still be detected with the CatWalk at 45 minutes whereas the variability of the von Frey measurement does not allow the detection of significant changes in the withdrawal threshold at that time point. The advantage of a higher sensitivity is closely linked to the second one: the reproducibility of the CatWalk-measure. The variability of CatWalk-based results is clearly lower than the variability in the von Frey results (Table 1). It results in lower number of animals needed, leading to compelling ethical as well as financial advantages. Such a good reproducibility of the results can first be explained by the computerized data of the CatWalk technique as compared to the handmade measuring of the von Frey withdrawal threshold. Next, it can also be explained by the amount of animals included in this study. Although the experimental group included 6 rats, only 4 of them could be correctly evaluated with the von Frey test. The CAR injection induced a spontaneous and almost incessant withdrawal of the ipsilateral paw in two of the experimental rats. Consequently, the stimulation of the ipsilateral paw with von Frey filaments was not possible and these two rats could not be included in the von Frey study. However, rats using only three paws can be used in CatWalk analysis. For instance, a non-used paw corresponds to a value of 0 for the CatWalk individual paw-related parameters included in this study. Hence, all rats were included into the CatWalk gait analysis. Finally, a third advantage of the CatWalk over the von Frey technique is the objectivity of the measurements. The CatWalk analysis is fully computerized and allows an objective measurement, independent to the experimenter. This contrasts highly with the relatively subjective assessment of a paw withdrawal response evoked by von Frey filament stimulation which highly depends on the experimenter. 


\section{Conclusion}

We can strongly recommend the CatWalk as sensitive, reproducible and objective technique to assess analgesic drug effects in experimental models of acute postoperative pain.

\section{Acknowledgment}

This study was performed within TREND (Trauma Related Neuronal Dysfunction), a knowledge consortium that integrates research CRPS type I, and is supported by a Dutch government grant (BSIK 03016). 


\section{References}

1. Gensel, J.C., et al., Behavioral and histological characterization of unilateral cervical spinal cord contusion injury in rats. J Neurotrauma, 2006. 23(1): p. 36-54

2. Hamers, F.P., et al., Automated quantitative gait analysis during overground locomotion in the rat: its application to spinal cord contusion and transection injuries. J Neurotrauma, 2001. 18(2): p. 187-201

3. Vrinten, D.H. and F.F. Hamers, 'CatWalk' automated quantitative gait analysis as a novel method to assess mechanical allodynia in the rat; a comparison with von Frey testing. Pain, 2003. 102(1-2): p. 203-9

4. Gabriel, A.F., et al., The CatWalk method: a detailed analysis of behavioral changes after acute inflammatory pain in the rat. J Neurosci Methods, 2007. 163(1): p. 9-16

5. Kulkarni, S.K. and V.P. Singh, Licofelone--a novel analgesic and anti-inflammatory agent. Curr Top Med Chem, 2007. 7(3): p. 251-63

6. Jones, C.K., S.C. Peters, and H.E. Shannon, Synergistic interactions between the dual serotonergic, noradrenergic reuptake inhibitor duloxetine and the non-steroidal anti-inflammatory drug ibuprofen in inflammatory pain in rodents. Eur J Pain, 2007. 11(2): p. 208-15

7. Rodrigues, A.R., et al., Participation of ATP-sensitive K+ channels in the peripheral antinociceptive effect of fentanyl in rats. Braz J Med Biol Res, 2005. 38(1): p. 91-7

8. Herrero, J.F. and R.E. Solano, The antinociceptive effect of the mu-opioid fentanyl is reduced in the presence of the alpha(2)-adrenergic antagonist idazoxan in inflammation. Brain Res, 1999. 840(1-2): p. 106-14

9. Lankhorst, A.J., et al., Effects of enriched housing on functional recovery after spinal cord contusive injury in the adult rat. J Neurotrauma, 2001. 18(2): p. 203-15

10. Radhakrishnan, R., S.A. Moore, and K.A. Sluka, Unilateral carrageenan injection into muscle or joint induces chronic bilateral hyperalgesia in rats. Pain, 2003. 104(3): p. 567-77

11. Min, S.S., et al., A novel method for convenient assessment of arthritic pain in voluntarily walking rats. Neurosci Lett, 2001. 308(2): p. 95-8

12. Coulthard, P., S.U. Simjee, and B.J. Pleuvry, Gait analysis as a correlate of pain induced by carrageenan intraplantar injection. J Neurosci Methods, 2003. 128(1-2): p. 95-102

13. Clarke, K.A., et al., Gait analysis in a rat model of osteoarthrosis. Physiol Behav, 1997. 62(5): p. 951-4

14. Coulthard, P., et al., Gait analysis as an objective measure in a chronic pain model. J Neurosci Methods, 2002. 116(2): p. 197-213

15. Winter, C.A., E.A. Risley, and G.W. Nuss, Carrageenin-induced edema in hind paw of the rat as an assay for antiiflammatory drugs. Proc Soc Exp Biol Med, 1962. 111: p. 544-7

16. Meert, T.F. and H.A. Vermeirsch, A preclinical comparison between different opioids: antinociceptive versus adverse effects. Pharmacol Biochem Behav, 2005. 80(2): p. 309-26

17. Pasero, C., Fentanyl for acute pain management. J Perianesth Nurs, 2005. 20(4): p. 279-84

18. Koppert, W., et al., The effects of intradermal fentanyl and ketamine on capsaicin-induced secondary hyperalgesia and flare reaction. Anesth Analg, 1999. 89(6): p. 1521-7

19. Eisenach, J.C., et al., Alfentanil, but not amitriptyline, reduces pain, hyperalgesia, and allodynia from intradermal injection of capsaicin in humans. Anesthesiology, 1997. 86(6): p. 1279-87

20. Li, X., M.S. Angst, and J.D. Clark, A murine model of opioid-induced hyperalgesia. Brain Res Mol Brain Res, 2001. 86(1-2): p. 56-62 


\section{Environmental housing affects the}

\section{duration of mechanical allodynia and}

the spinal astroglial activation in a rat model of chronic inflammatory pain A. F. Gabriel'1,2, M. A.E. Marcus', W. M.M. Honig', N. Helgers, E. A.J. Joosten ${ }^{1,2}$

'Department of Anesthesiology, Academic Hospital Maastricht, 6202 AZ Maastricht, the Netherlands 


\section{Abstract}

In this study, we aimed at investigating the effect of an enriched environment (EE) on the recovery from chronic inflammatory pain. Inflammatory pain was induced by the injection of $2 \mathrm{mg}$ of carrageenan (CAR) into the right knee of male Sprague-Dawley rats $(n=34)$. Rats were housed either singly (S-housed) or in an EE (EE-housed). The EE consisted of a large cage $(\mathrm{L} \times \mathrm{W} \times \mathrm{H}=2.0 \times 1.0 \times 0.8 \mathrm{~m})$ containing various attributes (e.g. running wheels, shelter house, climbing frame). Withdrawal response to von Frey filament was used to assess mechanical allodynia at days post-operative (DPO) -1, 1, 7, 14, 21 and 28. S-housed animals showed a marked tactile sensitivity in the ipsilateral paw from DPO1 to DPO21. Four weeks after the CAR-injection, S-housed rats were no longer allodynic. In contrast, EE-housed rats showed a significantly faster recovery: already at DPO21, they were no longer allodynic. In a first attempt to analyse the possible role of astroglial cells in the EE-induced effect, histological analysis at DPO21 was performed. Immunohistochemical staining of the spinal dorsal horn at L3-L5 indeed showed that spinal levels of astroglial activation are different between the two housing groups and therefore may play a role in the EE-induced effect on the duration of mechanical allodynia. In conclusion, our results showed that EE-housing results in a reduced duration of mechanical allodynia in chronic inflammatory pain in rats. Astroglial activation is suggested to be involved in this housing effect. 


\section{Introduction}

Chronic pain is the most common cause of long-term disability in middle-aged people in industrialized countries [1]. Studies on the effect of environment on patients recovery after surgery in general or more particularly on pain [2-4] have led to the development and empirical validation of various psychosocial approaches for treating chronic pain [5-7].

In experimental pain research, environmental enrichment (EE) models a part of the clinical rehabilitation environment of chronic pain patients. EE is described as 'a combination of complex inanimate and social stimulation' [8]. For rodents, the EE consists of a large cage containing various attributes (e.g. running wheels, shelter house, climbing frame, additional nesting material) where about ten animals are housed together $[9,10]$. Numerous studies indicate that such an enriched environment induces morphological and molecular changes in the brain. For instance, animals reared under enriched conditions display increased cortical mass $[11,12]$ and neurotransmitter levels $[13,14]$ and perform better on learning and memory tasks compared to rats reared in individual standard cages $[15,16]$. However, with respect to the effects of EE on pain sensitivity and perception, only a limited number of studies are available. Tall et al. (2008) have demonstrated that home cage conditions significantly affect the magnitude of inflammation-induced nociception. In addition, it was shown that EE-housing determines the duration and degree of reversal tactile allodynia in a severe spinal cord injury pain model, during which the neurotrophin Brain-Derived Neurotrophic Factor (BDNF) might play a neuroprotective role [17]. Finally, EE has been shown to significantly affect thermal sensitivity, using an operant orofacial pain assay [18].

The biological mechanism underlying the effect of housing on pain duration and/or magnitude is poorly described. Although studies on environmentally driven plasticity in the central nervous system (CNS) have traditionally focused on altered neuronal function, non neuronal cells are also known to be strongly influenced by EE. Astrocyte morphology and number have been reported to change if rats are reared in complex environments, with the changes depending both on the duration of housing as well as on the cortical layer in which the astrocytes reside [19-21]. Changes in astrocyte morphology are known to be correlated to modifications in synapse formation [22,23]. Based on this and as astrocytes are described as key players in the maintenance of inflammatory pain [24], we hypothesized that the EE could affect the pain process via an action on spinal astrocytes.

Therefore, this study attempted to investigate the effect of an EE on the recovery from carrageenan (CAR)-induced inflammatory pain in a rat model. Moreover, we suggested that spinal astrocytes are involved in the biological mechanism underlying this effect. Hence, histological analysis of the spinal dorsal horn will be performed at level L3-L5, three weeks after the induction of inflammation. 


\section{Material and methods}

\section{Animal housing and care}

A total of 34 male Sprague-Dawley rats weighing 250g was used. During the experiment animals were housed either in standard conditions (S-housed) or in an philippe.gabriel@saint-gobain.com enriched environment (EE-housed). S-housing consists in a standard cage $(\mathrm{L} \times \mathrm{W} \times \mathrm{H}=0.5 \times 0.2 \times 0.2 \mathrm{~m})$ containing a single tunnel and basic nesting material where animals are singly housed. Enriched environment consists of a large cage $(\mathrm{L} \times \mathrm{W} \times \mathrm{H}=2.0 \times 1.0 \times 0.8 \mathrm{~m})$ containing various attributes (e.g. running wheels, shelter house, climbing frame, additional nesting material) $[9,10]$ where animals are socially housed (about 10 rats per cage). All rats were housed under a normal $12 \mathrm{~h}$ light/12 h dark cycle. Food and water were provided ad libitum. This study was approved by the Ethics Committee on Animal Experiments of the University of Maastricht (DEC protocol no. 2006-032).

\section{Experimental design and Carrageenan-induced knee inflammation}

At first, the behavioral study was performed (total $n=24$ ). Prior to the injection, animals were housed in standard conditions ( $\mathrm{S}$-housing). At $\mathrm{t}=0$, all animals were injected intra-articularly with $2 \mathrm{mg}$ of $\lambda$-CAR (dissolved in $200 \mu \mathrm{l}$ of saline solution - $\mathrm{NaCl} 0.9 \%$ ) (Sigma-Aldrich, no.C3889) into the right knee. The injection was performed under anesthesia (inhaled isofluorane 2.5\%). Rats were allowed to recover from anesthesia for one day. At DPO 1, the behavioral test was performed and, immediately after, half of the rats were housed in an EE whereas the other half remained in the S-housing. Behavioral tests were performed preoperatively, at DPO1, 7, 14, 21 and 28. Rats were sacrificed at DPO28. Second, histological study was performed (total $n=10$ ). It was decided to analyse spinal astrocytes activation at DPO21, where behavioral results showed the major difference between the two groups. The experiment was repeated. Behavioral tests were performed at the same time points. At DPO21, animals were perfused and spinal cord tissues were further processed.

\section{Behavioral test}

Mechanical allodynia was assessed using the von Frey test by measuring paw withdrawal threshold in response to mechanical stimuli. Animals were placed in a plastic cage with a metal mesh floor, allowing them to move freely. They were placed in 
this cage 10 min prior testing to allow them to acclimatize to the environment. Filaments of various thicknesses (North Coast Medical, Inc., CA, USA) were applied to the mid-plantar surface of both rat hind paws. Each probe was applied to the foot until it just bent. Then the regularly calibrated stiffness corresponds to an applied force of $0.16,0.4,0.6,1.0,1.4,2.0,4.0,6.0,8.0,10,15,26,60$ and $100 \mathrm{~g}$. A minimum of three withdrawals of the tested paw out of five filament applications was considered as a positive response. Filaments were applied in ascending order, and the smallest filament that elicits a positive response was considered the threshold stimulus. Finally, the von Frey test was performed preoperatively and one day after the injection and from then on, once a week, up to 4 weeks.

\section{Histological study}

\section{Perfusion and tissue processing}

Five rats per experimental group were perfused on day 21 post-injection with $4 \%$ paraformaldehyde in $0.1 \mathrm{M}$ phosphate buffer ( $\mathrm{pH} 7.4$ ) after being anesthetized with a lethal dose of Nembutal $(0.10 \mathrm{ml} / 100 \mathrm{~g}$ of bodyweight). The spinal cord was removed, post-fixed overnight in the same fixative, then in $10 \%$ glucose overnight and finally stored in $25 \%$ glucose, at $4^{\circ} \mathrm{C}$, until further processing. The lumbar enlargement was selected, separated from the rest of the spinal cord, embedded in Tissue-Tec ${ }^{\circledast}$ and stored at $-80^{\circ} \mathrm{C}$. Using the cryostat, $25 \mu \mathrm{m}$-thick transverse sections were made of the lower part of the enlargement (L3 to L5) and mounted on formolgelatine-coated glass slides.

\section{Astroglial immunohistochemistry}

Astrocytes activation is described to be correlated with major cellular changes among which an increase in the Glial Fibrillary Acidic Protein (GFAP) [25]. In the present study, the intensity of GFAP-immunoreactive staining in the dorsal grey matter of L3 to L5 was analysed as a marker of astroglial activation, in every eighth transverse serial section. The sections were air-dried for 2 hours and washed successively in Tris-buffer saline (TBS, $0.1 \mathrm{M}$ ) with $0.2 \%$ Triton X-100 (TBS-T), pH 7.4, TBS and TBS-T again. A primary antibody either against GFAP (1:1600; rabbit anti-GFAP; DAKO was used overnight. After various wash-steps with TBS-T, TBS and TBS-T, either Cy3-conjugated donkey anti-rabbit (1:800; Sanbio) was used as a secondary antibody for 90 minutes at room temperature. Finally, the sections were washed three times with TBS and coverslipped with TBS/glycerol. 


\section{Analysis}

Immunostained sections were examined under a fluorescent microscope (Olympus Ax70). Photomicrographs were taken of the GFAP immunostained sections at a $10 \times$ and $20 \times$ objective respectively (and a $12.5 \times$ condenser) using a greyscale Fview camera. Photomicrographs were taken of both the ipsilateral and contralateral dorsal horns. Per animal between 16 and 24 photomicrographs were collected and analysed. Analysis was performed using the software program CellP ${ }^{\oplus}$, a soft imaging system. Importantly, the dorsal horns were divided into six laminae according to a rat spinal cord atlas [26]. Transparent schematic drawings were made according to the structure of the spinal cord as represented in this atlas. Placement of these drawings onto the photomicrographs was then performed to delineate laminae I to III as regions of interest (ROI) (see Illustration 1). The analysis was strictly performed in sections where the shape of the dorsal horn fitted with the schematic drawing from the atlas. The grey value mean of the GFAP stained sections was measured in each individual lamina. Laminae I to III on the contralateral side of the spinal cord served as the control.

\section{Statistics}

In the behavioral study, the effect of the CAR injection over time on the von Frey threshold was assessed through a repeated measure nonparametric test (Friedman test; $\mathrm{p}<0.05)$ with a Dunns correction, followed by Wilcoxon matched pairs test (for comparison with contralateral values at each time point $)(\mathrm{p}<0.05)$.

In the histological analysis, comparison between ispi- and contralateral values were statistically tested via paired t-test. Comparisons between the different housing groups were statistically tested by the mean of unpaired t-test.

All data are expressed as mean \pm S.E.M.

\section{Results}

All data (histological and behavioral) have been analysed by a blinded experimenter. No complications (i.e. more than $10 \%$ of the body weight or stop self-care) after injection of $2 \mathrm{mg}$ of carrageenan (CAR) in the right knee of the rats were noted $(n=34)$. In the behavioral study, five rats were excluded from the von Frey analysis (four rats in the S-housing and one rat in the EE) because of a constant withdrawal of the injured limb during the first twenty-four hours which did not allow a reliable von Frey measurement. 


\section{Enriched housing decreases the duration of mechanical allodynia}

The development of mechanical allodynia was assessed by the von Frey test. The mean pre-injection threshold noted for EE housed animals was $38.4 \mathrm{~g} \pm 7.6$ (Mean \pm S.E.M.) and $40.6 \mathrm{~g} \pm 14.3$ for the S-housed rats (Figure 1). The intra-articular injection of CAR into the right knee resulted in an overall decrease of the mechanical withdrawal threshold in both groups. At DPO1, von Frey withdrawal thresholds of the ipsilateral paw showed a major significant decrease to $1.5 \mathrm{~g} \pm 0.3$ in EE housed animals and to $1.7 \mathrm{~g} \pm 0.9$ in S-housed rats. In S-housed animals, a significant difference between ipsi- and contralateral paw withdrawal thresholds was noted at DPO7 ( $p<0.01)$, DPO $14(\mathrm{p}<0.01)$ and at DPO 21 ( $\mathrm{p}<0.05)$. At four weeks after CAR injection, a return to pre-injection levels was noted and no significant difference was observed between the von Frey withdrawal threshold of ipsi- $(41.8 \mathrm{~g} \pm 17.1)$ and contralateral $(57.0 \mathrm{~g} \pm 12.3)$ hind paws. In EE-housed animals, a significant difference between ipsi- and contralateral paw withdrawal thresholds was noted at DPO7 $(\mathrm{p}<0.01)$ and DPO $14(\mathrm{p}<0.01)$. In EE housed rats, 3 weeks after the CAR injection (DPO21), no significant difference was observed between the von Frey withdrawal threshold of ipsi- $(56.8 \mathrm{~g} \pm 10.8)$ and contralateral (75.1 $\mathrm{g} \pm 7.8)$ hind paws.

\section{Spinal astrocytes differ between the two housing groups}

Spinal astroglial response was studied at three weeks after the CAR-injection in EE and S-housed animals. DPO21 was chosen since, at that time-point, the EE-housed rats did not show any mechanical allodynia whereas S-housed rats still did. The effect of housing on the response of astrocytes in the dorsal horn of the spinal cord (L3 to L5) following a knee CAR-induced inflammation was studied using GFAP immunohistochemistry (Illustrations 1 and 2). The intensity of the staining was quantified using the CellP' program. S-housed rats demonstrated a significantly higher intensity of GFAP-immunoreactivity in the ipsilateral as compared to the contralateral side of the dorsal horn in lamina I (ipsi-grey value: 1748.9 a.u. \pm 70.0 ; contra-grey value: 1902.9 a.u. $\pm 65.4 ; \mathrm{p}<0.01$ ), in lamina II (ipsi-GV: 1415.0 a.u. \pm 48.9 ; Contra-GV: 1515.15 a.u. $\pm 51.9 ; \mathrm{p}<0.01$ ) and in lamina III (ipsi-GV: 1125.9 a.u. \pm 31.5 ; Contra-GV: 1222.4 a.u. $\pm 39.4 ; \mathrm{p}<0.01$ ) (Figure 2). In contrast, EE-housed rats did not show any difference in the intensity of the GFAP-immunoreactivity in laminae I, II and III, between the ipsilateral and contralateral side of the dorsal horn.

Comparison of EE- and S-housed rats showed that the values of the contralateral sides did not differ between the two groups (unpaired t-test, n.s.), neither for the ipsilateral sides (unpaired t-test, n.s.). 


\section{Discussion}

$\lambda$-CAR injection into tissues is known to induce inflammation. The CAR model (injection of CAR into the rat knee) is described as a model of acute inflammatory pain during the first $24 \mathrm{~h}$. A chronic inflammatory pain state is reached after one to two weeks that lasts up to 3 to 6 weeks, depending on the amount of CAR injected $[27,28]$. The present study demonstrated that EE housing as compared to S-housing after a CAR-induced inflammatory pain significantly reduces the duration of mechanical allodynia in rats. Quantitative histological analysis of the spinal dorsal horn layers I, II and III at DPO21 suggested astroglial cells to be involved.

Housing conditions are known to influence behavioral, hormonal and neuronal mechanisms, both in humans and rodents [8,29]. For instance, supplementing the standard home cage of rats with toys and other objects has been shown to increase the anti-nociceptive effects of opioid drugs [3], and improve the recovery after spinal cord injury $[9,17,30]$. In an inflammatory pain model using the injection of Freud's adjuvant into the left hindpaw, the degree of thermal hyperalgesia was shown to be significantly lower in rats housed in a supplemented home cage environment, as compared to rats housed in basic cages [31]. Thermal sensitivity to an operant orofacial nociception assay was also affected by housing conditions, such that rats living in supplemented home cages displayed significantly greater tolerance to a thermal stimulus as compared to rats housed in basic home cages (Rossi and Neubert, 2008). Therefore, the effects of EE housing in reduction of the duration of mechanical allodynia after CAR-induced inflammatory pain observed in this study
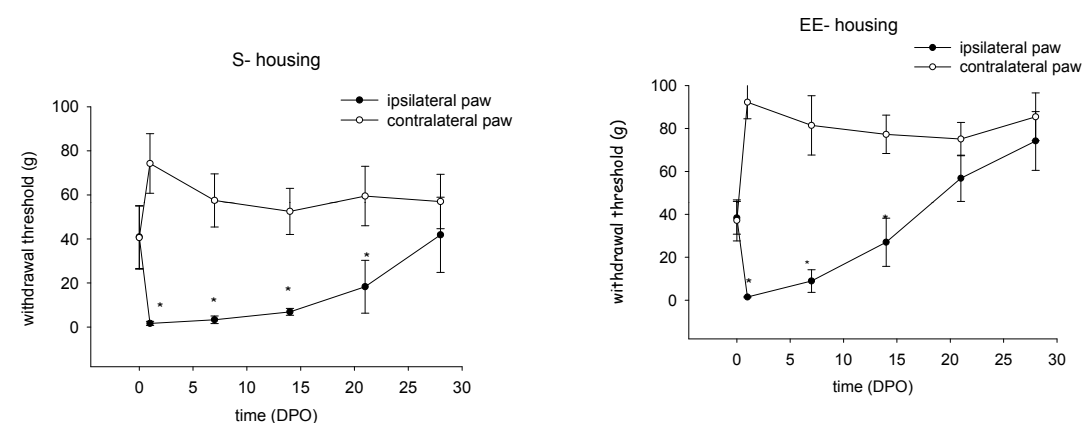

Figure 1: Influence of the housing on the development of mechanical allodynia: Time course of von Frey withdrawal threshold in a rat model of inflammatory pain. Measurements were taken pre-operatively and at DPO (Day Post-Operation) 1, 7, 14, 21 and 28. Data are expressed as mean \pm S.E.M. The symbol (*) indicates a significant difference between the ipsi- and the contralateral values of the rats hindpaws $(p<0.05)$. 
are in line with the reported data on the beneficial effects of housing on nociceptive responses. Nevertheless, the question remains what the underlying mechanism is of the beneficial effect of EE housing on nociceptive responses.

EE housing is known to induce various alterations in brain structure and function, including increasing the birth and maturation of new neurons into functional circuits $[8,32]$, enhancing the expression of molecules involved in neuronal signaling $[33,34]$ and promoting synaptic plasticity $[35,36]$. These changes can influence brain function and plasticity by modifying synaptic transmission, enhancing signaling between neuronal ensembles and strengthening neuronal circuits [29]. Not only neuronal cells are affected by EE housing but also glial cells. EE has been reported to enhance the generation of astroglia in adult rat neocortex after focal cortical ischaemia [37]. Nilsson and Pekny suggested that EE not only changes astrocytes morphology but at the same time affects and refines the functional relationship between the astrocytes and neurons [38].
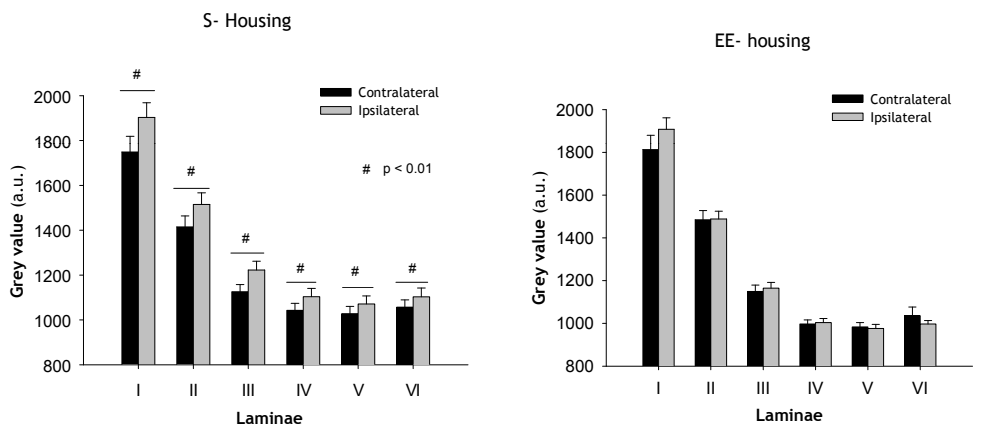

Figure 2: Influence of the housing on spinal GFAP-immunoreactivity, 21 days after the CAR injection: Intensity of the GFAP-immunoreactivity in the ipsilateral side (grey bars) and the contralateral side (black bars) of the rat spinal dorsal horn. The intensity of the grey value is quantified using the CellP ${ }^{\circledR}$ program and express in absolute values. Data are expressed as mean \pm S.E.M. The symbol (\#) indicates a significant difference between the ipsi- and the contralateral values of the grey intensity in the dorsal horn $(p<0.01)$.

Spinal glial cells are also known to be activated in response to inflammation or damage to peripheral tissues, peripheral nerves, spinal nerves or spinal cord $[24,39]$. The generally accepted view is that microglia are activated first and are involved in the onset of inflammatory pain whereas astrocytes are activated at later stages and are involved in the maintenance of pain [40]. Therefore, it was hypothesized that at DPO21, after a CAR injection into the right knee of an adult rat, only astroglial cells 
are involved in the pain modulation. From this, and according to the influence of housing conditions on astrocytes previously described, we studied the responses of astroglial cells in relation to the presence or absence of a mechanical allodynia in Sor EE-housed rats at DPO21. The analysis was focused on pain-relevant laminae (I, II, III) of the rat spinal dorsal horn. Whereas our behavioral results showed a clear difference between S- and EE-housed rats at DPO21, we first aimed to analyse the histological astroglial staining at this particular time point.

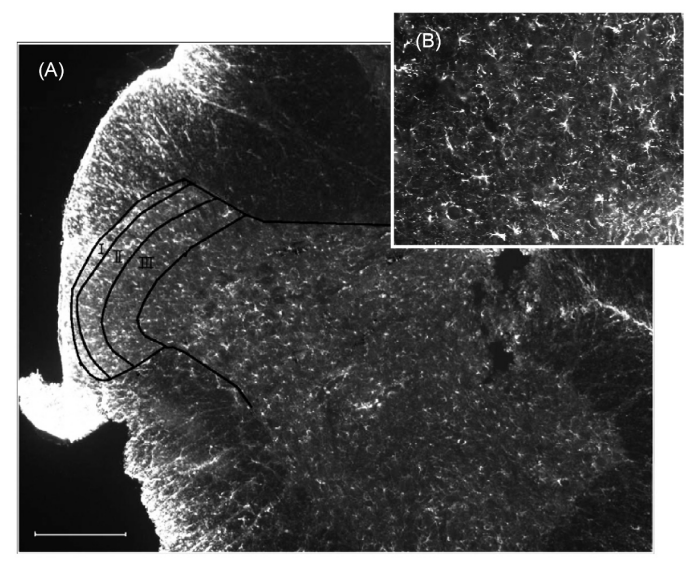

Illustration 1: GFAP staining in an EE-housed animal (A) schematic delimitation of the laminae I to III of the dorsal horn at L4 spinal level (Obj. × 40) (B) Obj × 200 .

A careful quantitative histological analysis of GFAP-immunoreactivity in laminae I, II or III of the ipsi- and contralateral dorsal horn of EE- and S-housed rats showed a difference in the ipsilateral/contralateral response of astroglial cells (i.e. of GFAP immunoreactivity) in both laminae I, II and III of the dorsal horn between the two housing groups. At DPO21, no mechanical allodynia was observed in EE-housed rats and, at the same time, no difference could be observed in the spinal astroglial response if ipsi- compared to the contralateral dorsal horn. In Shoused rats, a marked tactile hypersensitivity was still present at DPO21 according to the von Frey test, and here at the same time a clear astroglial response was noted in both lamina I, II and III of the ipsi- versus the contralateral side. In summary, our results showed a balance in the astroglial response between ipsi- and contralateral sides of the dorsal horn in rats housed in an EE (showing no mechanical allodynia anymore) but not in rats housed individually (still showing mechanical allodynia) at DPO21. 

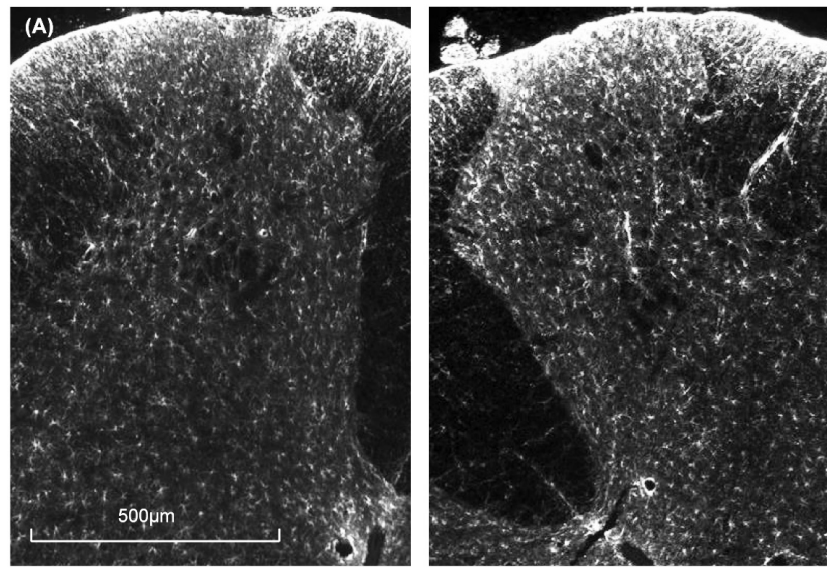

Illustration 2: Magnification (x40) photomicrographs of GFAP stained dorsal horn (L4 level) (A) in the contralateral side (B) at the ipsilateral side of the spinal cord in S-housed rats.

Although the involvement of astrocytes in the maintenance of inflammatory pain is less clear than in neuropathic pain [39], our observation that astrocytes are responsive in the maintenance stage of a peripheral inflammatory pain is in line with previous studies $[25,41]$. In this respect, Zhang and colleagues recently presented interesting data on the increase of IL- $1 \beta$-immunoreactive astrocytes in the ipsilateral side of the rat spinal cord following a CFA injection of the plantar surface of the right hind paw [42]. Their results suggested that astrocytes exert their actions by producing IL- $1 \beta$, which acts on nociceptive neurons by phosphorylating NR1 subunit of the NMDA-glutamate receptor and thereby maintaining the inflammatory pain.

Another interesting finding in our study is that no difference was observed in the (absolute) levels of spinal astroglial immunoreactivity between the EE and S-housed rats. This observation is intriguing in view of the previously mentioned well-established role of activated astrocytes in pain facilitation. In this respect, the emergent concept of the dual role of astroglial cells in pain facilitation and normalization may be important. Recent work from Svensson and colleagues suggested the existence of a mechanism through which spinal nonneuronal cells can participate in the regulation of sensory neuronal activity, not only through sensitization, but also by the normalization of pain signals [43]. Their experiments demonstrated that lipoxins attenuate nociception not only at the site of inflammation but also by a participation in the regulation of spinal pain processing through actions on astrocytes-expressed lipoxin receptors. This interaction between lipoxins and its astrocytic receptor is suggested to activate pathways for anti-inflammatory proresolving actions. They 
hypothesized that there is an elevation of lipoxins in the spinal cord during normal resolution of pain and, conversely, that a reduced capacity to produce peripheral and/or spinal lipoxins may lead to persistent pain. In this context, activated astrocytes are likely to play an important role in the resolution phase of inflammation by inducing anti-inflammatory cascades. Our results are in line with and indicate a role of EE-housing on astrocytes-mediated resolution phase of peripheral inflammatory state, where EE-housing would facilitate or fasten the process of resolution. Further experimental studies are needed to test these hypotheses.

Finally, and although we mainly focused on the difference of sensitivity between the ipsi- and contralateral paw following a CAR-injection, it is interesting to notice that the contralateral paw sensitivity is also affected over time. Indeed von Frey results show an increase in the contralateral paw withdrawal threshold (PWT) immediately after the CAR injection which remains constant until the end of the experiment. Here, it is hypothesized that the preoperative value of the von Frey results reflects a stress-induced hyperalgesia where stress would be due to the first handling-effect of the rats by the experimenter [44]. This effect takes place in both groups and is therefore not likely to influence the housing effect observed at DPO21 and the subsequent analysis of von Frey data.

In conclusion, our results showed that housing significantly affects the duration of inflammation induced mechanical allodynia. Astrocytes are suggested to be involved in the mechanism underlying the effect of $\mathrm{EE}$ on the recovery from chronic inflammatory pain. The clinical implications of our findings are extensive: a potential insight into designing better pain treatment environments, the reinforcement of non-pharmaceutical protocols to improve pain control in humans as well as the development of pharmaceutical targets in treatment of chronic inflammatory pain based on the potential role of astroglial cells in the resolution phase of inflammatory pain, as first suggested by Svensson and colleagues [43].

\section{Acknowledgment}

This study was funded by a grant from the Dutch Government, STW-NWO (ISO 41064) "ON/OFF switchable drug delivery in pain management" (to M.A.E.M. and E.A.J.J.). This study was performed within TREND (Trauma Related Neuronal Dysfunction), a knowledge consortium that integrates research on CRPS type I, and is supported by a Dutch Government grant (BSIK03016). 


\section{References}

1. van Tulder, M.W., B.W. Koes, and L.M. Bouter, A cost-of-illness study of back pain in The Netherlands. Pain, 1995. 62(2): p. 233-40

2. Ulrich, R.S., View through a window may influence recovery from surgery. Science, 1984. 224(4647): p. 420-1

3. Smith, M.A., P.A. Bryant, and J.M. McClean, Social and environmental enrichment enhances sensitivity to the effects of kappa opioids: studies on antinociception, diuresis and conditioned place preference. Pharmacol Biochem Behav, 2003. 76(1): p. 93-101

4. de Wied, M. and M.N. Verbaten, Affective pictures processing, attention, and pain tolerance. Pain, 2001. 90(1-2): p. 163-72

5. Bonifazi, M., et al., Changes in salivary cortisol and corticosteroid receptor-alpha mRNA expression following a 3-week multidisciplinary treatment program in patients with fibromyalgia. Psychoneuroendocrinology, 2006. 31(9): p. 1076-86

6. Flor, H., T. Fydrich, and D.C. Turk, Efficacy of multidisciplinary pain treatment centers: a meta-analytic review. Pain, 1992.49(2): p. 221-30

7. Morley, S., C. Eccleston, and A. Williams, Systematic review and meta-analysis of randomized controlled trials of cognitive behaviour therapy and behaviour therapy for chronic pain in adults, excluding headache. Pain, 1999. 80(1-2): p. 1-13

8. van Praag, H., G. Kempermann, and F.H. Gage, Neural consequences of environmental enrichment. Nat Rev Neurosci, 2000. 1(3): p. 191-8

9. Lankhorst, A.J., et al., Effects of enriched housing on functional recovery after spinal cord contusive injury in the adult rat. J Neurotrauma, 2001. 18(2): p. 203-15

10. Koopmans, G.C., et al., The assessment of locomotor function in spinal cord injured rats: the importance of objective analysis of coordination. J Neurotrauma, 2005. 22(2): p. 214-25

11. Rosenzweig, M.R., et al., Effects of environmental complexity and training on brain chemistry and anatomy: a replication and extension. J Comp Physiol Psychol, 1962. 55: p. 429-37

12. Bennett, E.L., M.R. Rosenzweig, and M.C. Diamond, Rat brain: effects of environmental enrichment on wet and dry weights. Science, 1969. 163(869): p. 825-6

13. O'Shea, L., et al., Neonatal 6-hydroxydopamine attenuates the neural and behavioral effects of enriched rearing in the rat. Eur J Pharmacol, 1983. 92(1-2): p. 43-7

14. Naka, F., et al., An enriched environment increases noradrenaline concentration in the mouse brain. Brain Res, 2002.924(1): p. 124-6

15. Kobayashi, S., Y. Ohashi, and S. Ando, Effects of enriched environments with different durations and starting times on learning capacity during aging in rats assessed by a refined procedure of the Hebb-Williams maze task. J Neurosci Res, 2002. 70(3): p. 340-6

16. Mohammed, A.K., et al., Environmental influence on behaviour and nerve growth factor in the brain. Brain Res, 1990. 528(1): p. 62-72 
17. Berrocal, Y., et al., Social and environmental enrichment improves sensory and motor recovery after severe contusive spinal cord injury in the rat. J Neurotrauma, 2007. 24(11): p. 1761-72

18. Rossi, H.L. and J.K. Neubert, Effects of environmental enrichment on thermal sensitivity in an operant orofacial pain assay. Behav Brain Res, 2008. 187(2): p. 478-82

19. Markham, J.A. and W.T. Greenough, Experience-driven brain plasticity: beyond the synapse. Neuron Glia Biol, 2004. 1(4): p. 351-363

20. Sirevaag, A.M. and W.T. Greenough, Differential rearing effects on rat visual cortex synapses. III. Neuronal and glial nuclei, boutons, dendrites, and capillaries. Brain Res, 1987.424(2): p. 320-32

21. Sirevaag, A.M. and W.T. Greenough, Plasticity of GFAP-immunoreactive astrocyte size and number in visual cortex of rats reared in complex environments. Brain Res, 1991.540(1-2): p. 273-8

22. Jones, T.A. and W.T. Greenough, Ultrastructural evidence for increased contact between astrocytes and synapses in rats reared in a complex environment. Neurobiol Learn Mem, 1996. 65(1): p. 48-56

23. Ullian, E.M., et al., Control of synapse number by glia. Science, 2001. 291(5504): p. 657-61

24. Watkins, L.R. and S.F. Maier, Glia: a novel drug discovery target for clinical pain. Nat Rev Drug Discov, 2003. 2(12): p. 973-85

25. Raghavendra, V., F.Y. Tanga, and J.A. DeLeo, Complete Freunds adjuvant-induced peripheral inflammation evokes glial activation and proinflammatory cytokine expression in the CNS. Eur J Neurosci, 2004. 20(2): p. 467-73

26. Paxinos G. and W. C., The rat brain in stereotaxic coordinates (4th ed.). Academic Press, San Diego, 1998

27. Radhakrishnan, R., S.A. Moore, and K.A. Sluka, Unilateral carrageenan injection into muscle or joint induces chronic bilateral hyperalgesia in rats. Pain, 2003. 104(3): p. 567-77

28. Gabriel, A.F., et al., The CatWalk method: Assessment of mechanical allodynia in experimental chronic pain. Behav Brain Res, 2008

29. Nithianantharajah, J. and A.J. Hannan, Enriched environments, experience-dependent plasticity and disorders of the nervous system. Nat Rev Neurosci, 2006. 7(9): p. 697-709

30. Koopmans, G.C., et al., Circulating insulin-like growth factor I and functional recovery from spinal cord injury under enriched housing conditions. Eur J Neurosci, 2006. 23(4): p. 1035-46

31. Tall, J.M., Housing supplementation decreases the magnitude of inflammation-induced nociception in rats. Behav Brain Res, 2008

32. Kempermann, G., E.P. Brandon, and F.H. Gage, Environmental stimulation of 129/SvJ mice causes increased cell proliferation and neurogenesis in the adult dentate gyrus. Curr Biol, 1998. 8(16): p. 939-42

33. Ickes, B.R., et al., Long-term environmental enrichment leads to regional increases in neurotrophin levels in rat brain. Exp Neurol, 2000. 164(1): p. 45-52

34. Rampon, C., et al., Effects of environmental enrichment on gene expression in the brain. Proc Natl Acad Sci U S A, 2000. 97(23): p. 12880-4

35. Artola, A., et al., Long-lasting modulation of the induction of LTD and LTP in rat hippocampal CA1 by behavioural stress and environmental enrichment. Eur J Neurosci, 2006. 23(1): p. 261-72

36. Green, E.J. and W.T. Greenough, Altered synaptic transmission in dentate gyrus of rats reared in complex environments: evidence from hippocampal slices maintained in vitro. J Neurophysiol, 1986. 55(4): p. 739-50 
37. Komitova, M., et al., Enriched environment after focal cortical ischemia enhances the generation of astroglia and NG2 positive polydendrocytes in adult rat neocortex. Exp Neurol, 2006. 199(1): p. 113-21

38. Nilsson, M. and M. Pekny, Enriched environment and astrocytes in central nervous system regeneration. J Rehabil Med, 2007.39(5): p. 345-52

39. McMahon, S.B., W.B. Cafferty, and F. Marchand, Immune and glial cell factors as pain mediators and modulators. Exp Neurol, 2005. 192(2): p. 444-62

40. Tanga, F.Y., V. Raghavendra, and J.A. DeLeo, Quantitative real-time RT-PCR assessment of spinal microglial and astrocytic activation markers in a rat model of neuropathic pain. Neurochem Int, 2004. 45(2-3): p. $397-407$

41. Obata, H., et al., Spinal glial activation contributes to postoperative mechanical hypersensitivity in the rat. J Pain, 2006. 7(11): p. 816-22

42. Zhang, R.X., et al., IL-1ra alleviates inflammatory hyperalgesia through preventing phosphorylation of NMDA receptor NR-1 subunit in rats. Pain, 2008. 135(3): p. 232-9

43. Svensson, C.I., M. Zattoni, and C.N. Serhan, Lipoxins and aspirin-triggered lipoxin inhibit inflammatory pain processing. J Exp Med, 2007. 204(2): p. 245-52

44. Donovan, J. and P. Brown, Handling and restraint. Curr Protoc Immunol, 2006. Chapter 1: p. Unit 13 



\section{Preoperative housing in an enriched environment significantly reduces}

the duration of post-operative pain

in a rat model of knee inflammation

A. F. Gabriel'1,3, M. A.E. Marcus', W. M.M. Honig', E. A.J. Joosten ${ }^{1,3}$

'Department of Anesthesiology, Academic Hospital Maastricht, 6202 AZ Maastricht, the Netherlands

${ }^{3}$ European Graduate School of Neuroscience (EURON), University of Maastricht, P.O. Box 616,6202 AZ, Maastricht, The Netherlands

Submitted 


\section{Abstract}

In human, attention has been paid lately to the influence of the environment on clinical postoperative pain. In experimental pain research, the main paradigm to study the effect of housing conditions is the enriched environment (EE). During EE housing, ten rats are housed in a large cage (i.e. social stimulation). This cage contains additional tools like running wheels (i.e. physical stimulation). So far, both clinical and experimental data have demonstrated the effect of an enriched postsurgical housing on the postoperative pain. In this study, it was our aim to investigate the influence of housing conditions prior to an operation on the development of postoperative pain, using a rat model of carrageenaninduced inflammatory pain. Four housing conditions were used: a 3-week pre-housing in standard conditions (S-) followed by a post-housing in an EE; a 3-week prehousing in EE followed by a postoperation S-housing; a pre- and post-housing in EE; a pre- and post- S-housing. The development of mechanical allodynia was assessed by the mean of the von Frey test, preoperatively and at day post-operative (DPO) 1, 3, 7, 10, 14, 17, 21, 24 and 28. Our results show that a 3 week pre-operative exposure to EE leads to a significant reduction in the duration of the carrageenan-induced mechanical allodynia, comparable with a post-operative exposure to EE. Strikingly, when rats were housed in EE prior to as well as after the carageenan injection into the knee, mechanical allodynia lasted only 2 weeks, as compared to 4 weeks in S-housed rats. 


\section{Introduction}

Effective postoperative pain management remains a significant clinical issue. Despite numerous clinical practice guidelines for pain management published throughout the last decennia by regulatory agencies and professional societies and the installment of acute pain services, the percentage and level of postoperative pain remains high [1]. Interestingly, lately, attention has been paid to the importance of the environment on patient and their recovery [2]. A new concept of 'healing environment' emerged, where not only the professionals would be the healing factor but also the building itself would have a positive influence on patients [3,4]. Such an environment includes both physical and social approaches: reducing noise in the patient's room [5], using adequate colors in patients room [6], or offering entertainment possibilities [7] have been shown to significantly reduce pain-relieve medication and hospital stay of postoperative patients.

In experimental pain research, the paradigm of enriched environment (EE) is used to study the influence of enriched conditions as compared to standard ones. $\mathrm{EE}$ is described as 'a combination of complex inanimate and social stimulation' [8]. For rodents, the EE consists of a large cage containing various attributes (e.g. running wheels, shelter house, climbing frame, additional nesting material) where about ten animals are housed together $[9,10]$. Previous studies indicate that such an enriched environment reduces nociception in experimental pain models. Tall et al. have demonstrated that home cage conditions significantly affect the magnitude of inflammation-induced nociception [11]. In addition, it was shown that EE-housing determines the duration and degree of reversal tactile allodynia in a severe spinal cord injury pain model, during which the neurotrophin Brain-Derived Neurotrophic Factor (BDNF) might play a neuroprotective role [12]. Finally, EE has been shown to significantly affect thermal sensitivity, using an operant orofacial pain assay [13].

Based on these compelling clinical and experimental findings about the beneficial effects of an enriched environment on the recovery from pain after an operation or injury, a major influence of the housing condition prior to the operation or injury can be hypothesized. Among other, orthopedic interventions can induce profound inflammatory postoperative response [14]. The knee inflammation model used in this investigation was induced by an intra-articular injection of carrageenan (CAR). This model is described as a model of acute inflammation during the first $24 \mathrm{~h}$, leading to local redness, edema and hyperalgesia. It develops into a model of chronic inflammation and long-lasting hyperalgesia after one to two weeks that lasts up to 3 to 8 weeks, depending on the amount of CAR injected $[15,16]$. Therefore, it was our aim in this study to investigate the effect of housing in an enriched environment prior to the induction of a CAR-induced knee inflammation on the recovery from pain in adult rats. In addition, comparison was made with a combined pre- and post-injection housing in an EE. 


\section{Methods}

\section{Design}

In this study, 40 male Sprague-Dawley rats (200-220g) (Charles River Laboratories) were used. During the experiment animals were housed either in standard housing conditions (S-housing) or in an enriched environment (EE). The standard housing conditions consist of a standard cage $(\mathrm{L} \times \mathrm{W} \times \mathrm{H}=0.5 \times 0.2 \times 0.2 \mathrm{~m})$ where rats are housed individually. Enriched environment consists of a large cage $(\mathrm{L} \times \mathrm{W} \times \mathrm{H}=2.0 \times 1.0 \times 0.8 \mathrm{~m})$ containing various attributes (e.g. running wheels, shelter house, climbing frame, additional nesting material) $[9,10]$. At $t=0$, all rats were injected intra-articularly with $2 \mathrm{mg}$ of $\lambda$-Carrageenan $(200 \mu \mathrm{l})$ (Sigma-Aldrich, no.C3889) into the rat right knee. Three weeks prior to the injection, all rats were assigned to one of the four experimental groups. 'S-housed' group (group\#1) was housed in S-housing conditions prior to and after the injection $(\mathrm{n}=10)$. 'EE-housed' group (group\#2) was housed in EE prior to and after the injection $(\mathrm{n}=10)$. 'postEE-housed' group (group\#3) was housed in S-housing conditions prior to the injection and in EE after the injection $(\mathrm{n}=10)$. 'preEE-housed' group (group\#4) was housed in EE prior to the injection and in S-housing conditions after the injection $(\mathrm{n}=10)$. All rats were housed under a normal $12 \mathrm{~h}$ light/12 h dark cycle. Food and water were provided ad libitum. This study was approved by the Ethics Committee on Animal Experiments of the University of Maastricht (DEC protocol no. 2008-061).

\section{Assessment of the mechanical allodynia: the von Frey test}

Mechanical allodynia was assessed using the von Frey test by measuring paw withdrawal threshold in response to mechanical stimuli. Animals were placed in a plastic cage with a metal mesh floor, allowing them to move freely. They were placed in this cage 10 min prior testing to allow them to acclimatize to the environment. Filaments of various thicknesses (North Coast Medical, Inc., CA, USA) were applied to the mid-plantar surface of both rat hind paws. Each probe was applied to the foot until it just bent. Then the regularly calibrated stiffness corresponds to an applied force of $0.16,0.4,0.6,1.0,1.4,2.0,4.0,6.0,8.0,10,15,26$, 60 and $100 \mathrm{~g}$. A minimum of three withdrawals of the tested paw out of five filament applications was considered as a positive response. Filaments were applied in ascending order, and the smallest filament that elicits a positive response was considered the threshold stimulus. Measurements were taken pre-operatively and at day post operation (DPO) 1, 3, 7, 10, 14, 17, 21, 24, 28. 


\section{Statistics}

The effect of CAR injection over time on the von Frey withdrawal threshold was assessed through a repeated measure nonparametric test (Friedman test; $\mathrm{p}<0.05$ ) with a Dunns correction, followed by Wilcoxon matched pairs test (for comparison with contralateral values).

\section{Results}

No complication after injection of $2 \mathrm{mg}$ of carrageenan in the right knee of the rats was noted $(n=40)$. One rat was excluded from the von Frey analysis (in the EE-housed group) due to permanent withdrawal of the injured limb which did not allow a reliable von Frey measurement during the first week.

\section{Effect of the housing on the development of mechani- cal allodynia}

The development of mechanical allodynia was assessed by the von Frey test. The mean ipsilateral pre-injection threshold noted for S-housed animals was $88.0 \mathrm{~g} \pm 6.1$ (mean \pm S.E.M.), $82.2 \mathrm{~g} \pm 6.1$ for EE-housed rats, $68.6 \mathrm{~g} \pm 6.9$ for postEE-housed rats and $88.0 \mathrm{~g} \pm 5.6$ for preEE-housed rats (Figure 1). No significant difference was noted between pre- values of the different groups. The intra-articular injection of carrageenan into the right knee resulted in an overall decrease of the mechanical withdrawal threshold in all groups. Already at DPO1, von Frey withdrawal thresholds of the ipsilateral paw showed a major significant decrease to $1.8 \mathrm{~g} \pm 0.6$ in S-housed animals (group\#1), to $1.0 \mathrm{~g} \pm 0.1$ in EE-housed animals (group\#2), to $1.0 \mathrm{~g} \pm 0.2$ in postEE-housed animals (group\#3) and to $1.4 \mathrm{~g} \pm 0.3$ in preEE-housed rats (group\#4). No significant difference was measured between withdrawal thresholds of the different groups at DPO1. In S-housed rats, a significant difference between ipsi- and contralateral paw withdrawal thresholds was noted up to DPO 24 $(\mathrm{p}<0.05)$. However, at four weeks after CAR injection, a return to pre-injection levels was noted and no significant difference was observed between the von Frey withdrawal threshold of ipsi- (59.8 $\mathrm{g} \pm 7.3)$ and contralateral $(62.8 \mathrm{~g} \pm 6.8)$ hind paws ( $p>0.05$ ). In EE-housed rats (group\#2), already 2 weeks after the CAR injection (DPO14), no significant difference was observed anymore between the von Frey withdrawal threshold of ipsi- $(45.6 \mathrm{~g} \pm 8.7)$ and contralateral $(60.7 \mathrm{~g} \pm 5.4)$ hind paws ( $p>0.05)$. In postEE-housed (group\#3), no significant difference could be observed between the von Frey threshold of the ipsi- $(55.1 \mathrm{~g} \pm 8.9)$ and contral- 
ateral $(68.0 \mathrm{~g} \pm 4.9)$ hind paws from DPO21 ( $\mathrm{p}>0.05)$. Similarly in preEE-housed rats (group\#4), no significant difference could be noted between ipsi- (52.2 g \pm 7.1 ) and contralateral $(64.6 \mathrm{~g} \pm 7.3)$ hind paws at DPO21 $(\mathrm{p}>0.05)$.

\section{Discussion}

Our results show that pre-housing in an EE significantly reduces the duration of postoperative pain. Our results confirm previous data showing that the duration of mechanical allodynia following a $2 \mathrm{mg}$ carrageenan (CAR)-knee injection in Shoused rats is 4 weeks [16]. Moreover, the combination of a pre- and post-operative EE-housing (EE-housed rats) leads to a striking reduction in the duration of postoperative pain, i.e. 2 weeks as compared to 4 weeks in S-housed rats.

The effect of a pre-operative housing on the duration of post-operative inflammatory pain as we have observed has never been described before in the clinic neither in experimental pain research. However, studies have been carried out with interventionist prehabilitation programs intending to prepare the patient and its body to withstand a stressful event such as an operation. Various clinical and experimental studies have been performed in which active specific exercise prior to surgery has led to significant reduction in post-operative pain and faster postsurgical recovery [17-19]. Nevertheless, the effects are often minor and contradictory results have also been published were preoperative exercise and education failed at improving postsurgical recovery $[20,21]$, whereas our findings show a significant major decrease of one week in the duration of postoperative inflammatory pain as compared to controls. Here, in our opinion, it is of importance to point out the major difference between the EE used in our experiment where physical and social activities are promoted but never forced and the prehabilitation programs where specific movement and actions are asked to be performed by the patients or animals. Such a difference between an interventionist prehabilitation program and non interventionist housing condition may explain the difference observed in the effects.

Likewise, the effect of the combination of both a pre- and post-operative EEhousing has also barely been described so far. However, the effect of housing supplementation 2 weeks prior to and 4 weeks after a CFA (Complete Freund Adjuvant)paw injection was recently studied [11]. Male Long-Evans rats were assigned to a housing treatment group, supplemented or not, and both cohorts were housed in groups of three rats per cage $(20 \mathrm{inch}$. long $\times 16 \mathrm{in}$. wide $\times 8.5$ in. tall). Housing supplementation consisted in the inclusion of additional objects to the home cage, with no difference in the size of the cage. Two weeks later, rats were injected with CFA in the left paw to induce a state of persistent inflammation [22]. Four weeks after the injection, the degree of thermal hyperalgesia was significantly lower in rats housed 
in supplemented home cage environment as compared to rats housed in basic environment. Similarly, a tendency to a lower mechanical allodynia in supplemented housed rats was observed but the difference was not significant [11]. These findings are in line with the beneficial effect of enriched housing conditions on nociceptive response described in the present study in EE-housed rats. Nevertheless, the major influence of the housing conditions prior to and after the injection found in our study as compared to the relative minor effects in Tall's study is most probably due to the quality of the environmental enrichment. Whereas in Tall' study the supplemented home cage only offers the inclusion of additional objects as compared to controls, our enriched environment consists of both a physical enrichment, with not only additional tools but also larger cages, together with a social enrichment as compared to the control housing.

The mechanism underlying the effect of housing conditions on the pain system is not yet understood. As previously reviewed, any injury provokes autonomic, endocrinal and immune processes as well as sensory signaling [23]. Through a common chemical language including neurotransmitters, peptides, endocannabinoids, cytokines and hormones, an ensemble of interdependent nervous, endocrine and immune processes operates in concert to cope with the injury. Therefore, many

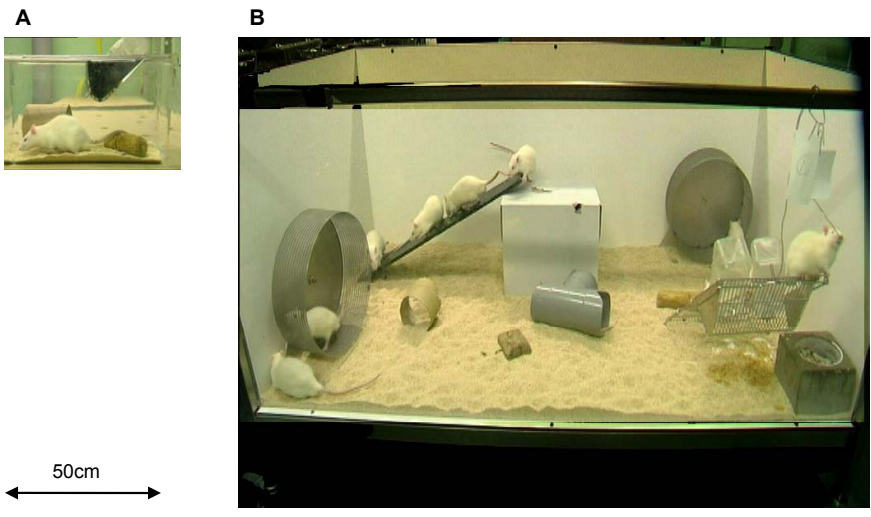

Illustration 1: Housing conditions (A) Standard housing (S-) consists of a standard cage $(\mathrm{L} \times \mathrm{W} \times \mathrm{H}=$ $0.5 \times 0.2 \times 0.2 \mathrm{~m}$ ) where animals are individually housed $(\mathbf{B})$ Enriched environment (EE) consists of a large cage $(\mathrm{LxWxH}=2.0 \times 1.0 \times 0.8 \mathrm{~m})$ containing various attributes (e.g. running wheels, shelter house, climbing frame, additional nesting material) where 10 animals are housed per cage.

Finally, it is interesting to notice that both the pre- and post-operative housing in EE (i.e. preEE housed rats and postEE-housed rats) result in a significant and comparable reduction of one week in the duration of the CAR-induced mechanical allodynia as compared to S-housed rats. This finding raises the question whether such a similar effect arises from similar underlying biological mechanisms. 

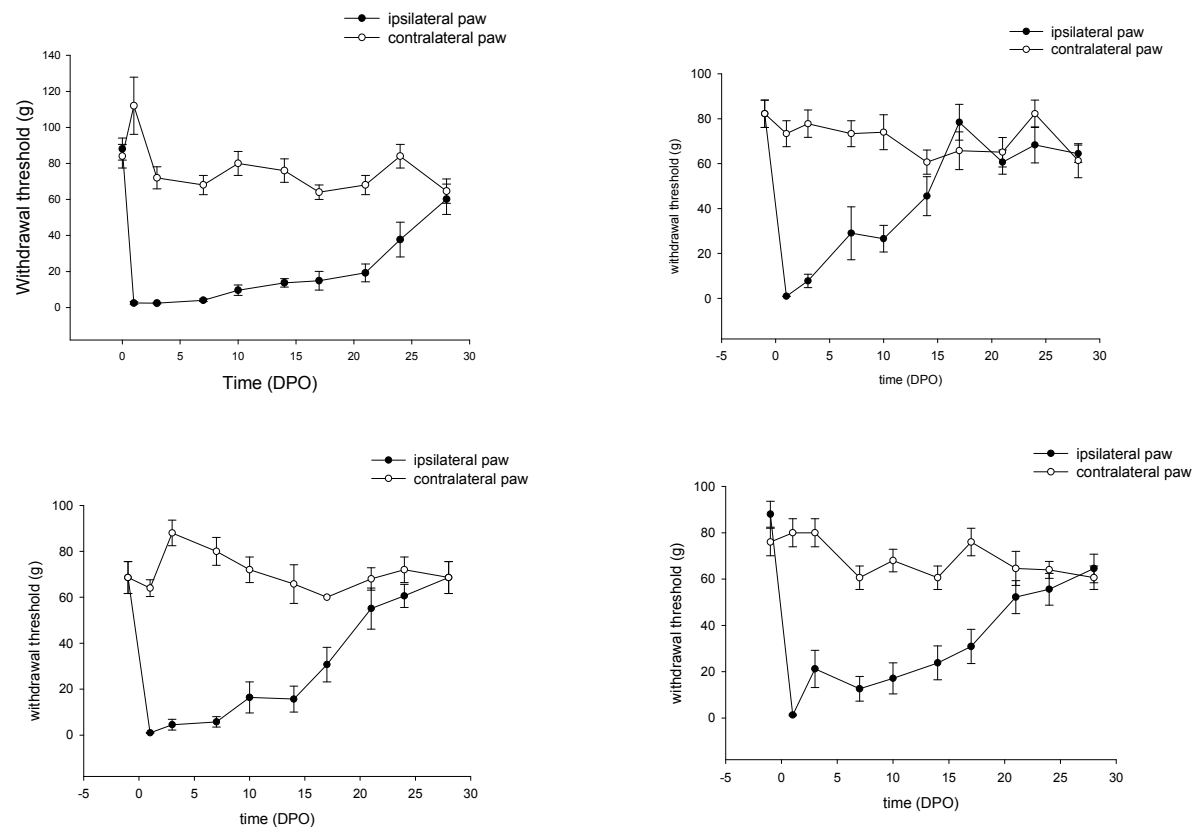

Figure 1: Von Frey analysis: time course of withdrawal thresholds to mechanical stimulus following an injection of 1\% CAR into the right knee with different housing conditions: (A) S-housed; (B) housed in an EE; (C) Shoused 3 weeks prior to the injection and in an EE after the injection; (D) housed in an EE prior to the injection and in S-housing conditions after the injection. Thresholds expressed in grams (g). Measurements were taken pre-operatively and at day post operation $1,3,7,10,14,17,21,24,28$. Data are presented as mean \pm S.E.M. The symbol $\left(^{*}\right)$ represents a significant difference in the ipsilateral and contralateral value $(p<0.05)$.

possible hypotheses can be formulated on the effect of housing conditions on the duration and intensity of inflammatory pain. The CAR-induced long-lasting hyperalgesia is a direct consequence of the inflammatory response to the injection of CAR into the rat knee rather than to a dysfunction of the nervous pain system. Consequently, we suggest that the observed effects in our study are most likely due to an effect of the housing on the immune system, possibly correlated to stress and the endocrine system. Clearly, these suggestions need further investigation. Nevertheless, the effect of the pre-operative EE-housing followed by a post-operative S-housing is intriguing (i.e. preEE housed rats). It is the first time that a pre-operative housing in $\mathrm{EE}$ is reported to result in a long-term effect (i.e. weeks after the CAR-injection) on the duration of hyperalgesia. Such a long term effect implies long term biological changes. Interestingly, no effect is found on the acute development of the mechanical allodynia caused by the CAR injection (at DPO1). The effect is exclusively 
observed as of one week after the injection (DPO7). In that respect, it is interesting to remember that the normal outcome of inflammatory program is successful resolution and repair of tissue damage [24]. The active process of 'catabasis', the return from inflammatory disease to health, seems to be programmed at the level of lipid mediators and protein mediators in both intracellular and extracellular events that are involved in damping inflammation and promoting its resolution [25]. The production of anti-inflammatory mediators is initiated through action of the immune system. More specifically, a mechanism has been suggested in which spinal activated astrocytes may play an important role in the resolution phase of inflammation through activation by lipoxin of anti-inflammatory cascades [26]. From these findings, it can be hypothesized that the decrease in inflammatory pain observed in pre-injection EE-housed rats is mediated through action on the immune-mediated resolution phase of inflammation and more particularly through action on astrocytes. Further studies are needed to test this hypothesis.

In conclusion, our results demonstrate that a pre-operative exposure to an EE leads to a significant reduction in the duration of a CAR-induced mechanical allodynia, comparable with a post-operative exposure to EE. Moreover, strikingly, when rats are housed in EE prior to and after the injection, the CAR-induced mechanical allodynia lasts 2 weeks, as compared with 4 weeks in S-housed rats. Our findings show that a preand post-operative enriched environment is important to reduce rat inflammatory pain. If this is also true in other species, like humans, should be investigated.

\section{Acknowledgment}

This study was funded by a grant from the Dutch Government, STW-NWO (ISO 41064) (to M.A.E.M. and E.A.J.J.). 


\section{References}

1. Miaskowski, C., Patient-controlled modalities for acute postoperative pain management. J Perianesth Nurs, 2005. 20(4): p. 255-67

2. Ulrich, R.S., View through a window may influence recovery from surgery. Science, 1984. 224(4647): p. 420-1

3. Long, R., Healing by design. Eight key considerations for building therapeutic environments. Health Facil Manage, 2001. 14(11): p. 20-2

4. Ananth, S., Building healing spaces. Explore (NY), 2008. 4(6): p. 392-3

5. Grumet, G.W., Pandemonium in the modern hospital. N Engl J Med, 1993.328(6): p. 433-7

6. Schweitzer, M., L. Gilpin, and S. Frampton, Healing spaces: elements of environmental design that make an impact on health. J Altern Complement Med, 2004. 10 Suppl 1: p. S71-83

7. Fry, W.F., Jr., The physiologic effects of humor, mirth, and laughter. JAMA, 1992.267(13): p. 1857-8

8. van Praag, H., G. Kempermann, and F.H. Gage, Neural consequences of environmental enrichment. Nat Rev Neurosci, 2000. 1(3): p. 191-8

9. Lankhorst, A.J., et al., Effects of enriched housing on functional recovery after spinal cord contusive injury in the adult rat. J Neurotrauma, 2001. 18(2): p. 203-15

10. Koopmans, G.C., et al., The assessment of locomotor function in spinal cord injured rats: the importance of objective analysis of coordination. J Neurotrauma, 2005. 22(2): p. 214-25

11. Tall, J.M., Housing supplementation decreases the magnitude of inflammation-induced nociception in rats. Behav Brain Res, 2009. 197(1): p. 230-3

12. Berrocal, Y., et al., Social and environmental enrichment improves sensory and motor recovery after severe contusive spinal cord injury in the rat. J Neurotrauma, 2007. 24(11): p. 1761-72

13. Rossi, H.L. and J.K. Neubert, Effects of environmental enrichment on thermal sensitivity in an operant orofacial pain assay. Behav Brain Res, 2008. 187(2): p. 478-82

14. Fanning, N.F., et al., Inhibition of neutrophil apoptosis after elective surgery. Surgery, 1999. 126(3): p. 527-34

15. Radhakrishnan, R., S.A. Moore, and K.A. Sluka, Unilateral carrageenan injection into muscle or joint induces chronic bilateral hyperalgesia in rats. Pain, 2003. 104(3): p. 567-77

16. Gabriel, A.F., et al., The CatWalk method: Assessment of mechanical allodynia in experimental chronic pain. Behav Brain Res, 2008

17. Arthur, H.M., et al., Effect of a preoperative intervention on preoperative and postoperative outcomes in low-risk patients awaiting elective coronary artery bypass graft surgery. A randomized, controlled trial. Ann Intern Med, 2000. 133(4): p. 253-62

18. Jaggers, J.R., et al., Prehabilitation before knee arthroplasty increases postsurgical function: a case study. J Strength Cond Res, 2007.21(2): p. 632-4

19. Kuphal, K.E., E.E. Fibuch, and B.K. Taylor, Extended swimming exercise reduces inflammatory and peripheral neuropathic pain in rodents. J Pain, 2007. 8(12): p. 989-97

20. D'Lima, D.D., et al., The effect of preoperative exercise on total knee replacement outcomes. Clin Orthop Relat Res, 1996(326): p. 174-82 
21. Beaupre, L.A., et al., The effect of a preoperative exercise and education program on functional recovery, health related quality of life, and health service utilization following primary total knee arthroplasty. J Rheumatol, 2004.31(6): p. 1166-73

22. Stein, C., M.J. Millan, and A. Herz, Unilateral inflammation of the hindpaw in rats as a model of prolonged noxious stimulation: alterations in behavior and nociceptive thresholds. Pharmacol Biochem Behav, 1988. 31(2): p. $455-51$

23. Chapman, C.R., R.P. Tuckett, and C.W. Song, Pain and stress in a systems perspective: reciprocal neural, endocrine, and immune interactions. J Pain, 2008. 9(2): p. 122-45

24. Serhan, C.N. and J. Savill, Resolution of inflammation: the beginning programs the end. Nat Immunol, 2005. 6(12): p. 1191-7

25. Bannenberg, G.L., et al., Molecular circuits of resolution: formation and actions of resolvins and protectins. J Immunol, 2005. 174(7): p. 4345-55

26. Svensson, C.I., M. Zattoni, and C.N. Serhan, Lipoxins and aspirin-triggered lipoxin inhibit inflammatory pain processing. J Exp Med, 2007. 204(2): p. 245-52 



\title{
Enriched environment and the
} recovery from inflammatory paín: social versus physical aspects and their interaction

\author{
A. F. Gabriel ${ }^{1,2}$, G. Paoletti ${ }^{3}$, D. Della Seta ${ }^{3}$, R. Panelli³, \\ M. A.E. Marcus ${ }^{1}$, F. Farabollini ${ }^{3}$, G. Carli ${ }^{3}$, E. A.J. Joosten ${ }^{1,2}$
}

'Department of Anesthesiology, Academic Hospital Maastricht, 6202 AZ Maastricht, The Netherlands ¿European Graduate School of Neuroscience (EURON), University of Maastricht, P.O. Box 616. 6202 AZ Maastricht, The Netherlands ${ }^{3}$ Department of Human Physiology, University of Siena, Siena, Italy

Submitted 


\section{Abstract}

In this study, we aimed at comparing the effect of the social versus the physical enrichment of the environment on inflammatory pain. In that purpose, a rat model of knee inflammation was used. Four housing conditions were investigated: a physically enriched environment (PE), a socially enriched environment (SE), an enriched environment (EE) (i.e. physically and socially enriched) and a restricted environment (RE) (i.e. non physically or socially enriched housing). Mechanical allodynia was assessed using the von Frey test preoperatively and at day post-operative (DPO) 1, 3, 7, 10, 14, 17, 21, 24 and 28 . Besides, anxiety was evaluated at DPO29, using the elevated plus maze test. Results show that RE housing resulted in a duration of mechanical allodynia of 4 weeks and of only 3 weeks in EE housing. Housing in a physically enriched environment (PE) also resulted in a reduction of the duration of mechanical allodynia of one week. Finally, if housed in a socially enriched environment (SE), the mechanical allodynia lasted for 3 weeks and an half. From these data, we conclude that both physical and social aspects of the environment are involved in the reduction of inflammatory pain duration, although the PE has a major effect than the $\mathrm{SE}$ in this experimental setting. Interestingly, an interdependent relationship was noted between the PE and SE. Moreover, no significant difference in the rat anxiety was measured between groups, suggesting that the pain outcomes are likely not biased by the mean of potential housing condition-induced anxiety. 


\section{Introduction}

The effect of the environment on pain sensitivity and perception can be important for the recovery of patients after surgery. In human, this is only investigated in a few studies [1-3]. This led to the development and empirical validation of various psychosocial and multidisciplinary approaches for treating chronic pain [4-6].

In experimental research, the main paradigm to study the effect of housing conditions is the enriched environment (EE). EE is described as a combination of complex inanimate and social stimulation' [7]. For rodents, the EE consists of a large cage containing various attributes (e.g. running wheels, shelter house, climbing frame, additional nesting material) where about ten animals are housed together [8, 9]. With respect to the effects of EE on pain sensitivity and perception, few studies have demonstrated it to lead to significant reduction of mechanical and/or thermal hyperalgesia in rat models of inflammation or spinal cord injuries [10-12].

Importantly, the EE used in experimental studies is known to consist of both physical and social aspects. Both the physical and the social aspects of the enrichment may significantly influence pain sensitivity and duration. Raber and Devor have demonstrated that social variables affect pain behavior in a neuropathic pain model [13]. Housing high (HA - High Autotomy) and low (LA - Low Autotomy) pain phenotype Wistar-derived rats housed in one cage induced a significant increase in autotomy of LA rats after nerve injury. The authors conclude that HA rats are apparently able to induce pain phenotype despite the powerful genetic predisposition of the LA rats and that social factors must be considered in behavioralrelated research and more particularly on pain perception. On the other hand, it was recently demonstrated that the degree of thermal hyperalgesia following Complete Freund Adjuvant (CFA)-induced paw inflammation was significantly lower in rats housed in a (physically) supplemented home cage environment, as compared to rats housed in basic cages [14].

Direct comparison between these different results for studying the effect of the social versus physical aspect of $\mathrm{EE}$ is very delicate since the effect of the environment on pain highly depends not only on the housing conditions but also on the type of injury, on the pain behavioral tests used as well as on the type and age of the animals. Therefore, this study was designed to compare the effect of the physical and social aspects of EE in a chronic inflammatory pain model in adult Sprague-Dawley rats, in order to determine to what extent each aspect differentially affects the duration of mechanical allodynia. Hence, we used four housing conditions in the present investigation: a physically enriched environment (PE), a socially enriched environment (SE), an enriched environment (EE) (i.e. physically and socially enriched) and a restricted environment (RE) (i.e. non physically or socially enriched housing) 
which served as a control. Pain outcome was measured during the development of mechanical allodynia using the von Frey test and an anxiety test was performed after recovery, by means of the elevated plus maze.

\section{Material and methods}

\section{Design}

The subjects were 48 male Sprague-Dawley rats (300-330 g) (Charles River Laboratories). Prior to the experiment, animals were individually housed in standard rat cages cage $(\mathrm{L} \times \mathrm{W} \times \mathrm{H}=0.5 \times 0.2 \times 0.2 \mathrm{~m})$ for one week. At $\mathrm{t}=0$, animals were anesthetized with Nembutal (Pentobarbital $40 \mathrm{mg} / \mathrm{kg}$ ) and injected intra-articularly into the right rat knee with $2 \mathrm{mg}$ of carrageenan (CAR) (Sigma-Aldrich, no.C3889). Animals were placed in their assigned housing immediately after surgery. Assessment of the development of mechanical allodynia was performed prior to the injection and at day post-operative (DPO) 1, 3, 7, 10, 14, 17, 21, 24 and 28. Anxiety test took place at DPO 29 in the 4 groups of treatment.

This study was approved by the local Ethics Committee on Animal Experiments of the University of Siena (9 September 2008).

\section{Housing}

All animals were housed with unlimited access to food and water, in a $12 \mathrm{~h}$ light: $12 \mathrm{~h}$ dark reversed cycle. Animals were assigned to one of four housing conditions (see Figure 1). In the physically enriched condition (PE), animals were individually housed in large three-floor cages $\left( \pm 220000 \mathrm{~cm}^{3} ; 72 \mathrm{~cm} \times 45 \mathrm{~cm} \times 69 \mathrm{~cm}\right)$ containing various inanimate attributes (tunnels, running wheel, extra nesting material) (total $n=10$ ). In the socially enriched condition (SE), animals were housed in groups of four rats in large standard rat cages $\left( \pm 40000 \mathrm{~cm}^{3} ; 38 \mathrm{~cm} \times 55 \mathrm{~cm} \times 18 \mathrm{~cm}\right)$ (total $\mathrm{n}=16$ ). In the enriched environment (EE), characterized by both physically and socially enriched conditions: groups of 4 rats were housed in large three-floor cages containing various inanimate attributes (tunnels, running wheel, extra nesting material) (total $n=12$ ). In the restricted environment (RE), animals were individually housed in large standard rat cages (total $n=10$ ). 


\section{Assessment of mechanical allodynia: the von Frey test}

Mechanical allodynia was assessed using the von Frey test by measuring paw withdrawal threshold in response to mechanical stimuli. Animals were placed in a plastic cage with a metal mesh floor, allowing them to move freely. They were placed in this cage 10 min prior testing to allow them to acclimatize to the environment. Filaments of various thicknesses (North Coast Medical, Inc., CA, USA) were applied to the midplantar surface of both rat hind paws. Each probe was applied to the foot until it just bent. Then the regularly calibrated stiffness corresponds to an applied force of 0.16 , $0.4,0.6,1.0,1.4,2.0,4.0,6.0,8.0,10,15,26,60$ and $100 \mathrm{~g}$. A minimum of three withdrawals of the tested paw out of five identical filament applications was considered as a positive response. Filaments were applied in ascending order, and the smallest filament that elicits a positive response was considered the threshold stimulus.

\section{Assessment of behavioral response in the Elevated Plus Maze}

Animals of the same cage were tested in sequence. The elevated plus-maze test, lasting 5 minutes, was carried out during the dark phase under dim red light, combined with a low, indirect white light. All animals were unfamiliar with the apparatus. The apparatus was thoroughly cleaned at the end of each test. All sessions were recorded with a video camera (Sony HI8) positioned above the apparatus. We subsequently scored the videotapes with software that allowed analysis of the frequency and duration of the behavioral parameters (Observer software, Noldus Information Technology).

The apparatus was made of black Perspex, and consisted of 2 open arms $(50 \times 10 \mathrm{~cm})$ and 2 closed arms (of the same size but with $34 \mathrm{~cm}$ high walls) extending from a central square platform $(10 \times 10)$. The arms were arranged so that those of the same type were opposite to each other. The apparatus was elevated $60 \mathrm{~cm}$ above the floor. Each animal was placed in the central square facing an open arm.

Standard spatiotemporal measures were recorded, i.e. the number of entries and the time spent in the open and closed arms (arm entry defined as $3 / 4$ of body into an arm) as well as the time spent in the central zone. From these data, the percentage of time spent in each of the three sections of the plus-maze (time/300 $\mathrm{s} \times 100$ ), the number of total entries (open + closed entries), and the percentage of open entries (open entries/total entries $\times 100$ ) were calculated.

In addition to these spatiotemporal measures, which yield separate measures of anxiety and locomotion $[15,16]$, behavioral parameters were recorded which are generally included in an enlarged version of the elevated plus-maze test in mice [17] and rats [18]. In particular, the frequency of head dips (exploratory head/ shoulder movement over sides of maze) and of stretched-attend posture (exploratory posture where the rat stretches forward and then retracts to its original posi- 
tion without locomoting forward) were observed. The frequency and duration of rearing, and duration of self-grooming and sniffing (olfactory exploration of the maze floor and walls, and occasional air sampling) were also scored. To analyze the thigmotactic cues in plus-maze exploration [17], stretched-attend postures and head dipping were further differentiated as "protected" (i.e., occurring on/from the relative security of the closed arms/center platform) or "unprotected". Data for the latter measures are, therefore, given as total scores and "percent protected" scores $[($ protected/total $) \times 100]$.

\section{Statistics}

The effect of the CAR injection over time on the von Frey threshold was assessed through a repeated measure nonparametric test (Friedman test; $\mathrm{p}<0.05$ ) with Dunns correction, followed by Wilcoxon matched pairs test (for comparison with contralateral values at each time point $)(\mathrm{p}<0.05)$. Elevated plus-maze behavior was analyzed by parametric one-way ANOVA, with treatment as the between-subjects factor. Variables that did not have a normal distribution were square-root transformed; if transformed data did not have homogeneous variance (Bartlett's test, $\mathrm{p}<0.05)$, they were analyzed with non-parametric Kruskal-Wallis ANOVA test. Multiple comparisons were performed using LSD or Mann-Whitney U tests [19].

\section{Results}

No complication after injection of $2 \mathrm{mg}$ of carrageenan in the right knee of the rats was noted $(n=48)$. Five rats were excluded from the von Frey analysis ( 2 rats in the $\mathrm{SE}$ and 2 rats in the EE) due to permanent withdrawal of the injured limb which did not allow a reliable von Frey measurement during the first week.

\section{Effect of the housing on the development of mechani- cal allodynia}

The development of mechanical allodynia was assessed by the von Frey test. The mean ipsilateral pre-injection threshold noted for EE housed animals was $55.6 \mathrm{~g} \pm 5.9$ (mean \pm S.E.M.), $88.0 \mathrm{~g} \pm 6.1$ for the RE housed rats, $56.7 \mathrm{~g} \pm 8.3$ for the PE housed rats and $68.6 \mathrm{~g} \pm 7.0$ for the SE housed rats (Figure 2). The intra-articular injection of carrageenan into the right knee resulted in an overall decrease of the mechanical withdrawal threshold in all groups. Already at DPO1, a significant difference was observed between the von Frey withdrawal thresholds (PWT) of both hind paws, with a decrease in the ipsi-PWT to $1.8 \mathrm{~g} \pm 0.5 \mathrm{in}$ EE housed animals, to $2.5 \mathrm{~g} \pm 0.7$ 
in RE housed animals, to $1.4 \mathrm{~g} \pm 0.4$ in PE housed animals and to $3.2 \mathrm{~g} \pm 0.9$ in SE housed rats. In RE housed rats, a significant difference between ipsi- and contralateral paw withdrawal thresholds was noted up to DPO $24(\mathrm{p}<0.05)$. At four weeks after CAR injection, a return to pre-injection levels was noted in RE rats and no significant difference was observed between the von Frey withdrawal threshold of ipsi- $(60.1 \mathrm{~g} \pm 8.4)$ and contralateral $(64.6 \mathrm{~g} \pm 7.0)$ hind paws $(\mathrm{p}>0.05)$. In EE housed rats, already 3 weeks after the CAR injection (DPO21), no significant difference was observed anymore between the von Frey withdrawal threshold of ipsi- (57.8 $\mathrm{g} \pm 7.8)$ and contralateral $(57.2 \mathrm{~g} \pm 6.0)$ hind paws $(\mathrm{p}>0.05)$. Similarly, in PE housed rats, no significant difference could be observed between the von Frey threshold of the ipsi- $(64.6 \mathrm{~g} \pm 6.2)$ and contralateral $(64.6 \mathrm{~g} \pm 6.1)$ hind paws from DPO 21 ( $\mathrm{p}>0.05)$. In SE housed rats, a significant difference was still noted between ipsi- $(39.1 \mathrm{~g} \pm 7.9)$ and contralateral $(63.3 \mathrm{~g} \pm 4.5)$ hind paws at DPO $21(\mathrm{p}<0.05)$. At DPO 24, no difference could be observed anymore $(\mathrm{p}>0.05)$.

\section{Effect of the housing on the Elevated Plus maze}

Analysis of data showed some statistical effects of the housing conditions on spatiotemporal measures in elevated plus-maze (Tab. 2). In particular, non parametric test revealed significant differences in closed $\operatorname{arm}\left(\mathrm{H}_{3,44}=7.84, \mathrm{p}=0.049\right)$ and total $\operatorname{arm}\left(\mathrm{H}_{3,44}=11.18, \mathrm{p}=0.011\right)$ entries. Post-hoc comparisons $(\mathrm{p}<0.05)$ showed that $\mathrm{PE}$ housed rats move to closed arms and to both closed and open arms more frequently than RE housed rats; moreover, EE housed rats entered in both arms more frequently than SE housed ones.
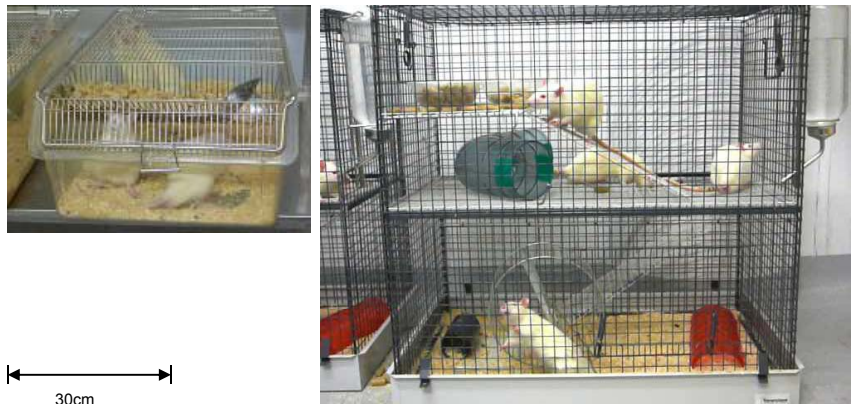

Figure 1: Housing conditions (A) small cage for individual [RE] and social [SE] housing in non-physically-enriched condition (volume of the cage $=40000 \mathrm{~cm}^{3}$ ) (B) large cage for individual [PE] and social [EE] enriched housing in physically-enriched condition (volume of the cage $=220000 \mathrm{~cm}^{3}$ ). 
(A)

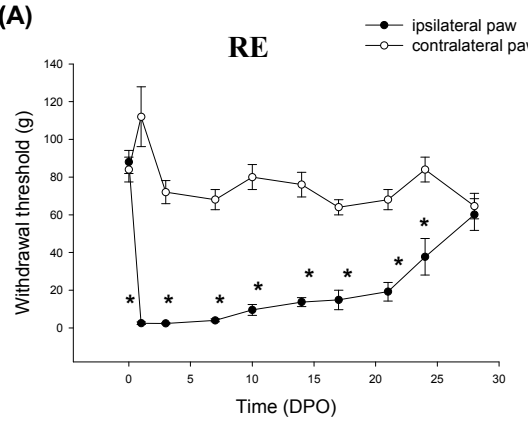

(C)

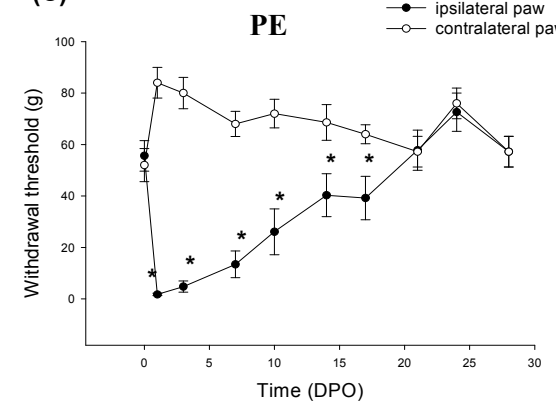

(B)

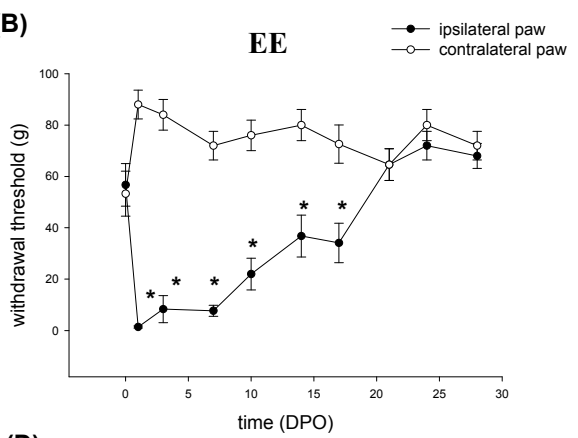

(D)

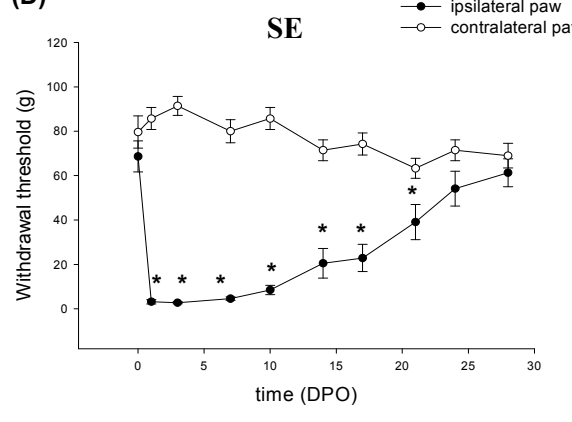

Figure 2: Withdrawal response to von Frey stimulus (A) in RE-housed rats (B) in EE-housed rats (C) in PEhoused rats (D) in SE-housed rats. Measurements were taken pre-operatively and at DPO (Day Post-Operation) $1,3,7,10,14,17,21,24$ and 28 . Values are expressed in gram (g). Data are expressed as mean \pm s.e.m. The symbol $(*)$ indicates values significantly different between ipsi- and contralateral paw $(p<0.05)$.

Parametric one-way ANOVA revealed differences between housing groups in central zone duration $\left(\mathrm{F}_{3,40}=3.09, \mathrm{p}=0.038\right)$ and in the percentage of time spent in the same zone $\left(\mathrm{F}_{3,40}=3.09, \mathrm{p}=0.038\right)$. Post-hoc analysis $(\mathrm{p}<0.05)$ showed that EE housed rats spent less time in central zone than RE and SE housed rats. No significant effect was found in other spatiotemporal measures as well as in behavioral parameters and thigmotactic cues (Table 1).

\section{Discussion}

Our results showed the impact of both the physical and the social aspects of EE on the duration of mechanical allodynia in a CAR-induced inflammation model. The duration of CAR-induced mechanical allodynia if rats were housed in a re- 
Table 1: Effect of the housing on the sensitivity to tactile allodynia: summary. EE = Enriched Environment; $\mathrm{RE}=$ Restricted Environment; PE = Physically enriched Environment; SE = Socially enriched Environment.

\begin{tabular}{cccc} 
Type of enrichment & Housing conditions & Comparison & $\begin{array}{c}\text { Reduction in duration of } \\
\text { mechanical allodynia } \\
(+\mathbf{1 / 2} \text { week; }++\mathbf{1} \text { week })\end{array}$ \\
\hline Enriched environment & Social / Large cage & EE vs RH & ++ \\
\cline { 2 - 4 } Physican enrichment & Individual & PE vs RH & ++ \\
& Social & EE vs SE & + \\
\cline { 2 - 4 } Social enrichment & Small cage & SE vS RH & + \\
& Large cage & EE vS PE & 0
\end{tabular}

stricted environment (RE) is 4 weeks. If housed in an enriched environment (EE) (physically and socially enriched), a reduction was noted and the CAR-induced mechanical allodynia lasted for 3 weeks.

Housing in a physically enriched environment (PE) also resulted in a reduction of the duration of mechanical allodynia of one week as compared to the RE. If housed in a socially enriched environment (SE), the CAR-induced mechanical allodynia lasted for 3 weeks and an half. Interestingly, the effect of PE on the duration of mechanical allodynia was comparable to the EE-effect. Furthermore, the effects of both the social and physical components of the enriched environment were more pronounced when the other component was limited (i.e. when housed in a small cage for the physical aspect and when housed individually for the social aspect) (Table 1). Finally, no difference could be observed in the anxiety levels between the four housing groups, using the elevated plus maze, at DPO29.

Recently, Tall reported the results of a study on the effect of a supplemented housing environment on the development of a CFA-induced paw inflammation in Long-Evans rats [14]. Rats were housed either in supplemented or basic housing condition and both cohorts were housed in groups of three rats per cage. Housing supplementation consisted in the inclusion of additional objects to the home cage, with no difference in the size of the cage. Two weeks later, rats were injected with CFA into the left hindpaw to induce a state of persistent inflammation [20]. Four weeks after the injection, the degree of thermal hyperalgesia was significantly lower in rats housed in a supplemented home cage environment as compared to rats housed in a basic environment. Similarly, a tendency to a lower mechanical allodynia in supplemented housed rats was observed but the difference was not significant [14]. These findings are in line with the beneficial effect of housing 
(A)

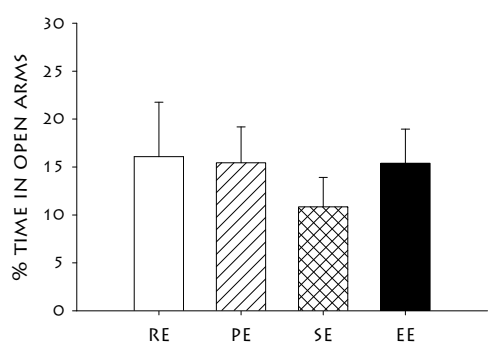

(C)

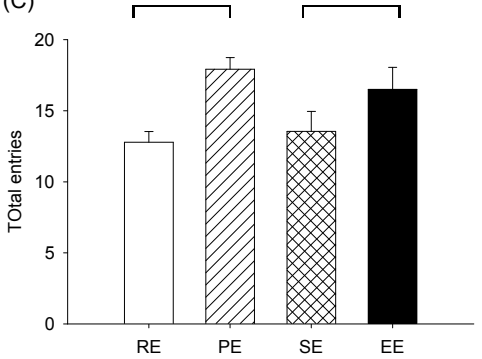

(B)

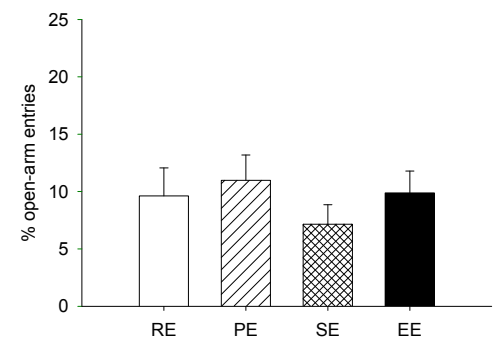

(D)

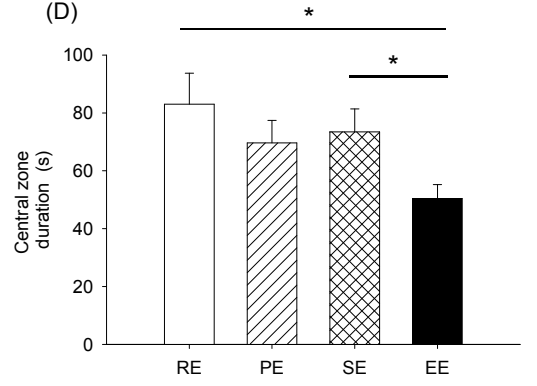

Figure 3: Effect of housing conditions on spatio-temporal parameters of the Elevated Plus Maze test: (A) \% time in open arms, (B) \% in open arms entries, (C) total entries and (D) time in central zone. Housing conditions are the following: $E E=$ Enriched Environment; $R E=$ Restricted Environment; PE = Physically enriched Environment; SE = Socially enriched Environment. Data are expressed as mean \pm s.e.m. The symbol $\left(^{*}\right)$ indicates values significantly different between ipsi- and contralateral paw $(p<0.05)$.

conditions on inflammatory pain response, as described in our study. Nevertheless, the difference in the statistical significance of the results between the two studies regarding the effect of the environment on the development of mechanical allodynia can most probably be explained by the difference in the housing supplementation. Indeed, whereas in Tall's study the supplemented home cage only offers the inclusion of additional objects, our physical enrichment additionally includes a larger cage. The decrease in the CAR-induced mechanical allodynia reported in our study is most probably due to enhanced physical activity. The opportunity to perform physical activity is significantly higher in our experimental setup as compared to Tall's housing enrichment. Similarly, extensive physical training such as nine days of swimming exercise (90min/day) decreased licking and flinching responses to intraplantar injection of formalin, as compared to non-exercised control animals [21]. 
Social housing variables and their influence on pain are likely to be intimately related to stress. Social isolation is known to be stressful for rats and mice [22,23], a potential basis for the exacerbation of pain sensitivity. Likewise, it was demonstrated that a single day of social isolation was enough to increase tail-shock-induced vocalizations. Besides, it was shown that morphine $(4 \mathrm{mg} / \mathrm{kg})$ was less effective in reducing vocalizations in animals housed in social isolation 3-4 days than in socially housed animals [24]. From these data, it was concluded that social contact can sustain endogenous analgesic processes in the body of the rat. On the other hand, social housing of rats generates the presence of dominant versus defeated rats and consequently, social defeat stress is also, in turn, a potential basis for the exacerbation of pain sensitivity. For instance, it was recently demonstrated that social defeat stress produces hyperalgesia in male Long-Evans rats [25]. Here it was suggested that a mutually facilitatory cross-regulation takes place between central pathways regulating stress and pain. In our study, a possible correlation between dominant/ defeated behavior and pain sensitivity was not studied. Nevertheless, no difference in the variation was observed in the von Frey withdrawal threshold between individually or socially housed rats (results not shown). We therefore conclude that the dominant/defeated behavior has a minimal impact on our data. Instead, our findings are in favor of the beneficial effect of social housing in rodents on stress and pain. Indeed, if housed in a small cage, rats developed a mechanical allodynia following CAR injection which lasted longer if housed individually (up to DPO 28) than if housed in groups (up to DPO 24).

Interestingly, we found an inter-dependent relationship between the social and the physical components of the EE (Table 1). The effect of the physical component on the duration of mechanical allodynia after the CAR injection depends on the social housing condition (whether animals are housed individually or in group). The same is noted for the social aspect whose effect depends on the quality of the physical environment (i.e. small versus large cage). The influence of the social aspect of the EE on the physical component regarding its effect on the duration of mechanical allodynia can be explained by an increased physical activity due to social interaction. If housed in a group, animals are sniffing and running around each other. One can easily assume that this socially increased physical activity has more impact in rats housed in small cage (in an impoverished environment) than in rats housed in large cages where physical activity is already promoted with additional tools and different floors to be explored in the cage, even if individually housed. The dependence of the social aspect of the EE on the physical component for its effect on the CAR-induced mechanical allodynia might be due to the physically induced level of stress. Recently, Mitsushima compared adult male Wistar-Imamichi rats housed in large cylindrical cage (diameter $=35 \mathrm{~cm})$ with rats housed in small cylindrical cage $($ diameter $=19 \mathrm{~cm})[26]$. From 
Table 2: Effect of housing condition on Elevated Plus-Maze parameters. The total duration of the test is 5 minutes. Data are presented as mean \pm s.e.m. RE: restricted environment; PE: physical enrichment; SE: social enrichment; EE: environmental enrichment. HD: head dipping; SAP: stretched-attend posture; $a=$ RE vS PE; $b=S E$ vs EE; $c=$ RE vs EE and SE vs EE; $p<0.05$.

\begin{tabular}{|c|c|c|c|c|c|c|c|c|c|c|c|c|}
\hline \multirow[b]{3}{*}{$\Omega$} & \multirow{2}{*}{\multicolumn{3}{|c|}{$\begin{array}{c}\mathrm{RE} \\
\mathrm{n}=9\end{array}$}} & \multirow{2}{*}{\multicolumn{3}{|c|}{$\begin{array}{c}\text { PE } \\
n=12\end{array}$}} & \multirow{2}{*}{\multicolumn{3}{|c|}{$\begin{array}{c}\text { SE } \\
\mathrm{n}=11\end{array}$}} & \multirow{2}{*}{\multicolumn{3}{|c|}{$\begin{array}{c}\text { EE } \\
n=12\end{array}$}} \\
\hline & & & & & & & & & & & & \\
\hline & 2.56 & \pm & 0.65 & 4.17 & \pm & 0.95 & 1.91 & \pm & 0.56 & 3.25 & \pm & 0.74 \\
\hline$\%$ open-arm entries & 9.62 & \pm & 2.44 & 10.98 & \pm & 2.21 & 7.15 & \pm & 1.57 & 9.88 & \pm & 1.90 \\
\hline Closed-arm entries & 10.22 & \pm & $0.83 a$ & 13.75 & \pm & 0.69 & 11.64 & \pm & 1.22 & 13.25 & \pm & 1.43 \\
\hline Total entries & 12.78 & \pm & $0.76 a$ & 17.92 & \pm & 0.82 & 13.55 & \pm & $1.40 \mathrm{~b}$ & 16.50 & \pm & 1.55 \\
\hline Open-arm duration & 48.25 & \pm & 17.05 & 46.33 & \pm & 11.23 & 32.53 & \pm & 9.23 & 46.17 & \pm & 10.70 \\
\hline Closed-arm duration & 168.73 & \pm & 16.09 & 184.03 & \pm & 14.40 & 194.07 & \pm & 10.34 & 203.44 & \pm & 11.88 \\
\hline Central zone duration & 83.01 & \pm & $10.71 c$ & 69.64 & \pm & 7.77 & 73.40 & \pm & $7.98 c$ & 50.39 & \pm & 4.83 \\
\hline Protected zone duration & 251.75 & \pm & 17.05 & 253.67 & \pm & 11.23 & 267.47 & \pm & 9.23 & 253.83 & \pm & 10.70 \\
\hline$\%$ time in open arms & 16.08 & \pm & 5.68 & 15.44 & \pm & 3.74 & 10.84 & \pm & 3.08 & 15.39 & \pm & 3.57 \\
\hline$\%$ time in closed arms & 56.24 & \pm & 5.36 & 61.34 & \pm & 4.80 & 64.69 & \pm & 3.45 & 67.81 & \pm & 3.96 \\
\hline$\%$ time in central zone & 27.67 & \pm & $3.57 \mathrm{c}$ & 23.21 & \pm & 2.59 & 24.47 & \pm & $2.66 \mathrm{c}$ & 16.80 & \pm & 1.61 \\
\hline$\%$ time in protected zone & 83.92 & \pm & 5.68 & 84.56 & \pm & 3.74 & 89.16 & \pm & 3.08 & 84.61 & \pm & 3.57 \\
\hline Rearing $(f)$ & 19.22 & \pm & 1.47 & 21.67 & \pm & 1.51 & 21.18 & \pm & 1.45 & 23.75 & \pm & 1.52 \\
\hline Rearing (d) & 39.57 & \pm & 4.51 & 36.82 & \pm & 2.67 & 34.64 & \pm & 2.45 & 36.20 & \pm & 3.66 \\
\hline Self-grooming (d) & 3.76 & \pm & 1.80 & 4.91 & \pm & 1.33 & 7.56 & \pm & 1.71 & 8.94 & \pm & 2.68 \\
\hline Sniffing (d) & 209.60 & \pm & 8.76 & 207.85 & \pm & 9.22 & 212.77 & \pm & 9.12 & 214.96 & \pm & 6.24 \\
\hline $\mathrm{HD}(f)$ & 9.67 & \pm & 1.84 & 13.17 & \pm & 2.12 & 10.00 & \pm & 1.08 & 10.42 & \pm & 1.41 \\
\hline $\operatorname{SAP}(f)$ & 0.22 & \pm & 0.15 & 0.25 & \pm & 0.13 & 0.36 & \pm & 0.15 & 0.67 & \pm & 0.28 \\
\hline HD in protected zone (f) & 4.89 & \pm & 0.75 & 7.00 & \pm & 0.94 & 6.00 & \pm & 0.93 & 4.65 & \pm & 0.63 \\
\hline SAP in protected zone $(f)$ & 0.22 & \pm & 0.15 & 0.33 & \pm & 0.18 & 0.45 & \pm & 0.20 & 1.08 & \pm & 0.47 \\
\hline
\end{tabular}


this experiment, it was suggested that restriction of the environment space could be considered as stressful likewise, in our study, if we consider the social housing having a beneficial effect on the duration of mechanical allodynia through a reduction of stress, one can assume that the impact of the social housing will be higher if rats are more stressed, i.e. if housed in a restricted environmental space.

In this study, the data show that the CAR-induced mechanical allodynia lasts for 3 weeks if rats are housed in PE and for 3 weeks and an half if housed in SE. On one hand, these data suggest that the physical activity has more impact on the duration of CAR-induced mechanical allodynia than the social component. On the other hand, it raises the question whether the social enrichment as designed in this study is optimal. The setup of the SE has been designed on a literature-based standard description [27, 28]. Nevertheless, a most favorable design may be developed that would allow a more pronounced effect to be observed. Further studies are needed to study alternative SE-setups.

Finally, the anxiety test demonstrates that 4-week housing in either RE, EE, PE or SE conditions did not influence the rat anxiety level at DPO 29. Indeed, no significant difference was measured between groups for the values of entries and time spent in open arms. This finding suggests that the housing conditions do not influence the rat anxiety level and therefore it was concluded that the outcomes from the pain behavioral test are likely not biased by the mean of a hypothetical housing condition-induced anxiety. Interestingly, statistical analysis showed some effects in elevated plus-maze test with respect to spatiotemporal measures. These effects can be interpreted on the basis of previous factor analytic studies on plus-maze in mice [17] and rats [18]. Both PE and EE animals entered the total arms more often than RE and SE groups, respectively. In these comparisons, the physical enrichment seems to be the main factor influencing the 'activity' of experimental animals. It is likely that previous physical experience facilitates the capacity to explore new areas, in particular the closed arms (closed arm entries, PE > RE). Lastly, EE housed animals spent less time in the central zone of the apparatus with respect to RE and SE groups. This measure may reflect a 'decision making' factor related to approach/ avoid conflict [29]; from central area only, animals can take a binary decision on whether to move and towards which arm. Animals must be able to detect the characteristics of the arms through sensory processes, and under the combined influence of physical and social experience the EE housed group seems to respond more quickly to such stimuli.

All together, our data demonstrate that both the physical and social aspects of the rat environment influence the development of CAR-induced inflammatory pain and that these two aspects are inter-dependent. Studies in humans are needed to confirm clinical implications of these findings. 


\section{Acknowledgment}

This study was funded by a grant from the Dutch Government, NWO-Senter (ISO 41064; to M.A.E.M. and E.A.J.J.). The authors thank Pr Carli and Pr Farabollini (University of Siena, Italy) for the financial support of the experiment. 


\section{References}

1. de Wied, M. and M.N. Verbaten, Affective pictures processing, attention, and pain tolerance. Pain, 2001. 90(1-2): p. 163-72

2. Smith, M.A., P.A. Bryant, and J.M. McClean, Social and environmental enrichment enhances sensitivity to the effects of kappa opioids: studies on antinociception, diuresis and conditioned place preference. Pharmacol Biochem Behav, 2003. 76(1): p. 93-101

3. Ulrich, R.S., View through a window may influence recovery from surgery. Science, 1984. 224(4647): p. $420-1$

4. Flor, H., T. Fydrich, and D.C. Turk, Efficacy of multidisciplinary pain treatment centers: a meta-analytic review. Pain, 1992. 49(2): p. 221-30

5. Morley, S., C. Eccleston, and A. Williams, Systematic review and meta-analysis of randomized controlled trials of cognitive behaviour therapy and behaviour therapy for chronic pain in adults, excluding headache. Pain, 1999. 80(1-2): p. 1-13

6. Bonifazi, M., et al., Changes in salivary cortisol and corticosteroid receptor-alpha mRNA expression following a 3-week multidisciplinary treatment program in patients with fibromyalgia. Psychoneuroendocrinology, 2006. 31(9): p. 1076-86

7. van Praag, H., G. Kempermann, and F.H. Gage, Neural consequences of environmental enrichment. Nat Rev Neurosci, 2000. 1(3): p. 191-8

8. Koopmans, G.C., et al., The assessment of locomotor function in spinal cord injured rats: the importance of objective analysis of coordination. J Neurotrauma, 2005. 22(2): p. 214-25

9. Lankhorst, A.J., et al., Effects of enriched housing on functional recovery after spinal cord contusive injury in the adult rat. J Neurotrauma, 2001. 18(2): p. 203-15

10. Tall, J.M., Housing supplementation decreases the magnitude of inflammation-induced nociception in rats. Behav Brain Res, 2008

11. Berrocal, Y., et al., Social and environmental enrichment improves sensory and motor recovery after severe contusive spinal cord injury in the rat. J Neurotrauma, 2007. 24(11): p. 1761-72

12. Rossi, H.L. and J.K. Neubert, Effects of environmental enrichment on thermal sensitivity in an operant orofacial pain assay. Behav Brain Res, 2008. 187(2): p. 478-82

13. Raber, P. and M. Devor, Social variables affect phenotype in the neuroma model of neuropathic pain. Pain, 2002. 97(1-2): p. 139-50

14. Tall, J.M., Housing supplementation decreases the magnitude of inflammation-induced nociception in rats. Behav Brain Res, 2009. 197(1): p. 230-3

15. Graeff, F.G., C.F. Netto, and H. Zangrossi, Jr., The elevated T-maze as an experimental model of anxiety. Neurosci Biobehav Rev, 1998. 23(2): p. 237-46

16. Hogg, S., A review of the validity and variability of the elevated plus-maze as an animal model of anxiety. Pharmacol Biochem Behav, 1996. 54(1): p. 21-30

17. Rodgers, R.J. and N.J. Johnson, Factor analysis of spatiotemporal and ethological measures in the murine elevated plus-maze test of anxiety. Pharmacol Biochem Behav, 1995. 52(2): p. 297-303 
18. Farabollini, F., S. Porrini, and F. Dessi-Fulgherit, Perinatal exposure to the estrogenic pollutant bisphenol A affects behavior in male and female rats. Pharmacol Biochem Behav, 1999. 64(4): p. 687-94

19. Zar, J.H., Biostatistical analysis. Prentice-Hall International, 1984. London

20. Stein, C., M.J. Millan, and A. Herz, Unilateral inflammation of the hindpaw in rats as a model of prolonged noxious stimulation: alterations in behavior and nociceptive thresholds. Pharmacol Biochem Behav, 1988. 31(2): p. 455-51

21. Kuphal, K.E., E.E. Fibuch, and B.K. Taylor, Extended swimming exercise reduces inflammatory and peripheral neuropathic pain in rodents. J Pain, 2007. 8(12): p. 989-97

22. Wiberg, G.S. and H.C. Grice, Long-Term Isolation Stress in Rats. Science, 1963. 142: p. 507

23. Ferrari, P.F., et al., Interindividual variability in Swiss male mice: relationship between social factors, aggression, and anxiety. Physiol Behav, 1998. 63(5): p. 821-7

24. Panksepp, J., Brief social isolation, pain responsiveness, and morphine analgesia in young rats. Psychopharmacology (Berl), 1980. 72(1): p. 111-2

25. Marcinkiewcz, C.A., et al., Social defeat stress potentiates thermal sensitivity in operant models of pain processing. Brain Res, 2008

26. Mitsushima, D., et al., Rats living in small cages respond to restraint stress with adrenocortical corticosterone release but not with hippocampal acetylcholine release. Psychoneuroendocrinology, 2003. 28(4): p. 574-83

27. Dobrossy, M.D. and S.B. Dunnett, Environmental enrichment affects striatal graft morphology and functional recovery. Eur J Neurosci, 2004. 19(1): p. 159-68

28. Sharp, J.L., et al., Stress-like responses to common procedures in male rats housed alone or with other rats. Contemp Top Lab Anim Sci, 2002. 41(4): p. 8-14

29. Cruz, A.P., F. Frei, and F.G. Graeff, Ethopharmacological analysis of rat behavior on the elevated plus-maze. Pharmacol Biochem Behav, 1994. 49(1): p. 171-6 


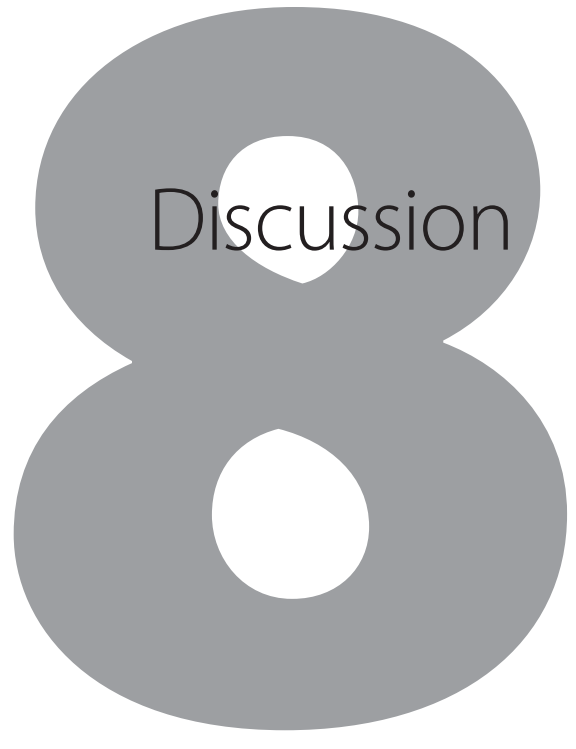


Pain assessment in experimental models is still in a relative immature state. Most techniques focus on pain assessment based on reflex responses. Future research should be focused on the development of behavioral analysis involving high level cerebral processing, e.g. operant tests, and approaches which more closely mimic how the experimental animal handles pain. In view of the use of more clinically relevant assessment techniques for analysis of behavioral changes after induction of inflammatory pain, we addressed the first research question of this thesis: Can the CatWalk method be used for objective assessment of pain-induced gait changes in a rat model of inflammatory pain?

In chapter 2, our results show that the CatWalk allows objective analysis of gait changes (i.e. intensity of the paw print and duty factor parameters) which at the same time parallel the development of mechanical hyperalgesia as assessed by the von Frey test. This, however, is restricted to the first 48 hours, i.e. the acute phase of inflammation. As a proof of principle, in chapter 4, subcutaneous injection of Fentanyl, an opioid analgesic drug, leads to a significant decrease in the CAR-induced changes of gait parameters during the acute phase of inflammation. These results confirm the causality of mechanical hyperalgesia in the observed CatWalk-based gait changes. Interestingly, in chapter 3, as of one week after the CAR injection, the CatWalk parameters intensity of the paw print and duty factor do no longer differ from pre-operative values whereas at the same time the von Frey withdrawal threshold indicates a mechanical hyperalgesia which lasts up to 4 weeks post-injection.

All together, we first conclude that the CatWalk is a potent and objective tool to assess pain-induced gait changes in correlation with mechanical hyperalgesia in the acute phase of the CAR-induced knee inflammation model. Nevertheless, its use needs to be carefully restricted to identified experimental pain models. Indeed, our results suggest that, as of one week after CAR injection, animals do no longer adapt their gait to the pain and that the phenomenon of habituation takes place. In the absence of gait changes in the chronic phase of the inflammation, the CatWalk gait analysis is no longer of use. Therefore, the CatWalk technique is not included in the following chapters of the thesis since they mainly focus on the effect of housing in an EE on the chronic phase of the inflammation model. Second, the results in chapters 2 and 4 related to the acute inflammatory pain response indicate that a simultaneous use of both von Frey and CatWalk tests to assess pain in experimental pain research will result in a better and more reliable picture of the whole pain experience in the rat. In the current need of experimental research to unravel the various aspects of pain [1], a combination of reflexive tests, operant tests and spontaneous behavior (i.e. gait) analysis could be beneficial in drug screening where still lots of drugs show a major effect in experimental pain models but do not reach the expec- 
tations when used in human [2-4]. From this point of view, further investigations including the use of different concentrations of Fentanyl and of other analgesic drugs such as NSAID's on CatWalk parameters in experimental pain models would be valuable in order to confirm the pertinence of the use of the CatWalk technique in analgesic drug pre-screening.

The second and main part of this thesis focused on the influence of the environment on the recovery from inflammatory pain. In experimental research, the paradigm of Enriched Environment (EE) provides greater social and physical interactions for rodents. Animals are socially housed in a large cage $(\mathrm{L} \times \mathrm{W} \times \mathrm{H}=2.0 \times 1.0 \times 0.8 \mathrm{~m})$ including extra nesting materials, tunnels and running wheels, as compared with standard housing $(\mathrm{S}-)$ were animals are singly housed in standard cage $(\mathrm{L} \times \mathrm{W} \times \mathrm{H}$ $=0.5 \times 0.2 \times 0.2 \mathrm{~m}$ ). To date, only few studies have been performed to investigate the effect of the environment on pain sensitivity and perception [5-7]. Hence, we raised the following clinically-relevant questions:

\section{- Does housing in an enriched environment enhance the recovery from in- flammatory pain?}

\section{- What is the differential effect of the two main components of an enriched environment on the recovery from inflammatory pain: physical versus so- cial enrichment?}

In chapter 5, we first aimed at studying the effect of post-operative housing in an EE on the duration of mechanical allodynia after a CAR-induced knee inflammation. We observed a three-week long lasting hyperalgesia in EE-housed rats as compared to four weeks in S-housed rats. Besides, histological analysis of the spinal dorsal horn suggests the astrocytes to be involved in the mechanism underlying the effect of environment on recovery from CAR-induced inflammatory pain. Moreover, in chapter 6, we studied the effect of EE housing prior to the induction of inflammation in order to examine the importance of the timing in the exposure to an EE. Our results demonstrate that a pre-injection exposure to EE leads to a similar reduction in the duration of mechanical allodynia as compared to a post-injection exposure. Even more striking is the effect of a combined exposure to EE pre- and post-injection on the duration of mechanical allodynia. A pre- and post-injection EE-housing leads to a 2-week reduction of the inflammatory pain as compared with S-housed rats. Such a significant reduction of duration of inflammatory pain is remarkable, especially for a non pharmaceutical approach. Finally, in chapter 7, we compared the effect of a socially enriched environment (SE) with a physically enriched environment (PE). Based on the von Frey withdrawal threshold, we observed that both 
SE and PE influence the duration of inflammatory pain, and that their effects are interdependent. Interestingly, PE induced a more pronounced effect than the SE in this experimental model of inflammatory pain.

All together, these compelling results indicate a major effect of the environment on the development of inflammatory pain. Housing in an EE prior to and/or after the induction of inflammation showed remarkable effects. Furthermore, our results emphasize the importance of both the physical and social components of housing conditions on the recovery from inflammatory pain.

Based on these results several future lines of research can be suggested. Importantly, the biological mechanism underlying the effect of the environment on the recovery from inflammatory pain definitely needs to be further investigated. In chapter 5 , we focused on spinal astrocytes activation as a first step in understanding the underlying biological mechanism. A difference in the balance between ipsi- and contralateral GFAP-immunoreactivity in the spinal dorsal horn suggests astrocytes to be part of the environmentally-driven reduction in CAR-induced inflammatory pain duration. This hypothesis is supported by the work of Svensson and colleagues which showed a key role of activated astrocytes in lipoxine-mediated resolution phase (i.e. the return to a normal state) of the inflammation [8]. Hence, an extended study of the development of spinal GFAP-immunoreactivity over time is first needed. Furthermore, if astrocytes are described as key players in maintaining persistent pain, it is important to notice that microglia are known to play a major role in its induction [9]. Therefore, study of the activation of microglia in the spinal dorsal horn in earlier stages of the inflammation (e.g. during the first week) may give some more insight on the environmentally-driven early activation of the resolution phase of inflammation. All together, our results raise the general question of how the EE could accelerate the resolution phase of peripheral inflammation. In this respect, a presumable general hypothesis is the influence of EE on the tripartite hypothalamic-pituitary-adrenal (HPA) axis/immune system/ pain system (Illustration 1). This hypothesis is based on the following evidences:

1. The HPA axis, a major endocrine regulating pathway, and glucocorticoids (GC) levels have been described to be influenced by both physical activity and social interactions [10-12]. Direct evidences between an EE and HPA axis are lacking but EE-housing has clearly been shown to reduce stress in animals $[13,14]$ and is therefore highly expected to influence the activity of the HPA axis.

2. HPA axis and GC levels influence the immune system at several levels among which are the expression of macrophages, the production of proinflammatory 
cytokines, and the antibody response to immune challenges [15]. In the current study, such an influence of the HPA axis on the immune system is expected to be directly related to the development and duration of inflammation and to its subsequent inflammatory pain.

3. Direct influence of the HPA axis on pain is also expected. First, GC receptors are found both on neurons and glial cells [16-18] and therefore both cell types activity are likely to be affected by changes in GC level and HPA axis activity. Second, a direct influence of the HPA axis on pain is likely to arise from the already described interaction of the HPA axis with the limbic system areas hippocampus, amygdala and prefrontal cortex [19] since these areas are known to be involved in the stressinduced pain modulation [20]. As most of these cortical areas involved in pain are connected to brainstem sites such as the periaqueductal grey matter [21], descending pathways are likely to be involved in this mechanism. Hence, in preliminary histological analyses, we studied the levels of serotonin (5-HT) and noradrenalin (NA) in the spinal dorsal horn of EE- and S-housed rats. No significant difference could be measured but a clear tendency to increased 5-HT and NA levels in the ipsilateral dorsal horn of the EE-housed rats was observed (results not shown). Complementary analysis and study of their respective receptors expression in the dorsal horn are still needed.

Based on these evidences, the HPA axis can be hypothesized to play a major role in the early activation of the resolution phase of inflammation in EE-housed rats. Nevertheless, it is interesting to notice that GC affect many aspects of the immune function and their effects are difficult to characterize [15]. Rather than being uniformly immunosuppressive, GC variably modulate the immune system. Indeed, there are

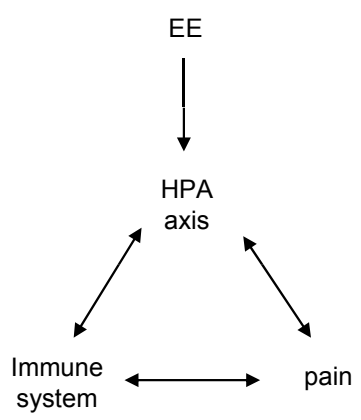

Illustration 1: Suggested partial mechanism underlying the EE-driven reduction in the duration of inflammatory pain. 
two major factors which confer selectivity of action of glucocorticoids on the immune system: the relative cellular expression of type I and/or type II adrenal steroid receptor subtypes, and whether or not the glucocorticoids are able to access the receptors within a given cell. The global effect of GC on the immune system therefore depends on many different parameters and is complex to predict. Further investigations will be needed to separately characterize the effect of long term EE-housing on HPA axis activity and GC levels, on immune system as well as on spinal neurons and glia activities in order to understand the exact role of EE on the early activation of the resolution phase from a peripheral inflammatory state.

Next to the HPA system, the hypothetical environmentally-driven regulation of the immune and pain systems may also involve other mechanisms such as the sympathetic nervous system (SNS) and opioid receptors [22-24]. However, Sluka et al. have demonstrated a minor involvement of SNS in the CAR-induced knee inflammation [25]. Based on these observations, SNS is not expected to play a major role in this model.

As mentioned in chapter 6 , it can be expected that although a pre- and a post-inflammation EE-housing induce a similar reduction in the duration of the inflammatory pain, the mechanism underlying these effects might not be the same. Therefore, separate studies of pre- and post-injection exposure to EE are needed to investigate potential differences in the underlying biological mechanisms.

In any case, the emergence of psychoneuroimmunology (PNI), concerned with the relations between nervous, immune and endocrine systems, would certainly be of valuable help [26]. PNI could lead to a better understanding, and possibly foreseeing, of the complex interactions between psychological processes, neural and immune systems and the influence of the environment on this tripartite.

To the author's view, it is worth mentioning that in the current work, the EE has been considered as a positive and enriched situation as compared to standard housing conditions. Another way to think about this 'environmental influence' issue could be as follows: rats being social animals, EE comes down to "standard" rat housing condition whereas the individual housing used in our work may be considered as a stressful, negative condition. The interpretation of the results would then be that a CAR knee injection leads to a three-week mechanical allodynia in the "standard" EE-housing. In case of stressful environment such as singly housed rats in a poor physical environment, an extension of one week is observed in the duration of mechanical allodynia as assessed by the von Frey test. Such an interpretation of our results still fits with our hypothetical mechanism where the stressful environment 
of individual housing would act on the HPA axis to finally induce the immune system and extend the duration of inflammation. Indeed, housing in impoverished conditions has been shown to induce depression-like behavior [27] which has been linked to markers of activated immune cells and increase concentrations of proinflammatory cytokines [28]. These markers may result in turn in increased pain response and/or duration.

Clinical implications of these results have to be further investigated. Human studies are needed to confirm or not the outcomes of the present work in view of the effect of the environment on the recovery of patients from inflammatory postoperative pain. Nevertheless, all together, our results would be in favor of the development of new hospital designs such as the so-called Healing Environment, where not only the professionals are the healing factor but also the experience of the building itself. In these newly designed buildings, attention is paid to both the physical and social aspects [29]. The concept is based on the observation that technological progresses in modern medicine have lead to austere, antiseptic smelling and unaesthetic buildings where almost no place to nature or natural compounds is found and which have negative impact on the patient's recovery.

Pointing out the importance of the environment on the development of inflammatory pain, the present work highlights the idea that the development of high-tech medical technologies may be an infertile development if we do not consider their consequences on patients' environment. Switching from hospital sites to our overall environment in Western societies, the same conclusion can be drawn: technological improvements have profoundly changed our every-day environment and our relationship to it. Indeed, the concept of progress has always been driving our societies [30]. In our industrialized countries, technological progress leads to more comfort, more material possession, saving time and providing new entertainment [31]. These developments can be double-edged [32]. Improving living conditions in health, hygiene, education and welfare for instance greatly contributed to increase human wellbeing [30]. But on the other hand, technological development of society is also blamed to induce profound structural changes such as urbanization, increased population and transcultural problems. Such factors are responsible for the development of psychosocial and psychosomatic diseases such as alcohol and drugs abuse, overweight and emotional stress [33].

The speed and urge of technological development observed during the last century did not allow the society to step back and to be aware of the environmental changes that accordingly occurred neither of the consequences of these changes on human physical and psychological well-being. Therefore, before claiming any overall ben- 
eficial effect of technological progress on humankind, not only is the quality of the newly developed knowledge and technologies to be considered but also the changes induced by these new technologies in our every-day environment and the influence of these changes on human welfare. Indeed, the importance of the environment on healing processes, as highlighted in the current thesis, and the clinical relevant literature available to date both support the value of an appropriately 'enriched environment', properly balanced between technological improvements and humanity. 


\section{References}

1. Ekblom, A. and P. Hansson, When is "pain" appropriate? Pain, 1993. 55(3): p. 403

2. Vierck, C.J., P.T. Hansson, and R.P. Yezierski, Clinical and pre-clinical pain assessment: are we measuring the same thing? Pain, 2008. 135(1-2): p. 7-10

3. Cooper, B.Y. and C.J. Vierck, Jr., Vocalizations as measures of pain in monkeys. Pain, 1986. 26(3): p. 393-407

4. Morgan, D., et al., Evaluation of prescription opioids using operant-based pain measures in rats. Exp Clin Psychopharmacol, 2008. 16(5): p. 367-75

5. Tall, J.M., Housing supplementation decreases the magnitude of inflammation-induced nociception in rats. Behav Brain Res, 2009. 197(1): p. 230-3

6. Berrocal, Y., et al., Social and environmental enrichment improves sensory and motor recovery after severe contusive spinal cord injury in the rat. J Neurotrauma, 2007. 24(11): p. 1761-72

7. Rossi, H.L. and J.K. Neubert, Effects of environmental enrichment on thermal sensitivity in an operant orofacial pain assay. Behav Brain Res, 2008. 187(2): p. 478-82

8. Svensson, C.I., M. Zattoni, and C.N. Serhan, Lipoxins and aspirin-triggered lipoxin inhibit inflammatory pain processing. J Exp Med, 2007. 204(2): p. 245-52

9. Watkins, L.R. and S.F. Maier, Glia: a novel drug discovery target for clinical pain. Nat Rev Drug Discov, 2003. 2(12): p. 973-85

10. Serra, M., et al., Social isolation-induced changes in the hypothalamic-pituitary-adrenal axis in the rat. Stress, 2005. 8(4): p. 259-64

11. Glasper, E.R. and A.C. Devries, Social structure influences effects of pair-housing on wound healing. Brain Behav Immun, 2005. 19(1): p. 61-8

12. Campbell, J.E., et al., Voluntary wheel running initially increases adrenal sensitivity to adrenocorticotrophic hormone, which is attenuated with long-term training. J Appl Physiol, 2009. 106(1): p. 66-72

13. Chamove, A.S., Cage design reduces emotionality in mice. Lab Anim, 1989. 23(3): p. 215-9

14. Ilin, Y. and G. Richter-Levin, Enriched environment experience overcomes learning deficits and depressivelike behavior induced by juvenile stress. PLoS ONE, 2009. 4(1): p. e4329

15. McEwen, B.S., et al., The role of adrenocorticoids as modulators of immune function in health and disease: neural, endocrine and immune interactions. Brain Res Brain Res Rev, 1997. 23(1-2): p. 79-133

16. Bohn, M.C., et al., In vitro studies of glucocorticoid effects on neurons and astrocytes. Ann N Y Acad Sci, 1994. 746: p. 243-58; discussion 258-9, 289-93

17. Crossin, K.L., et al., Glucocorticoid receptor pathways are involved in the inhibition of astrocyte proliferation. Proc Natl Acad Sci U S A, 1997. 94(6): p. 2687-92

18. De Nicola, A.F., et al., Regulation of gene expression by corticoid hormones in the brain and spinal cord. J Steroid Biochem Mol Biol, 1998. 65(1-6): p. 253-72

19. Herman, J.P., et al., Limbic system mechanisms of stress regulation: hypothalamo-pituitary-adrenocortical axis. Prog Neuropsychopharmacol Biol Psychiatry, 2005. 29(8): p. 1201-13

20. Ji, G. and V. Neugebauer, Differential effects of CRF1 and CRF2 receptor antagonists on pain-related sensitization of neurons in the central nucleus of the amygdala. J Neurophysiol, 2007.97(6): p. 3893-904 
21. Jasmin, L., et al., Rostral agranular insular cortex and pain areas of the central nervous system: a tracttracing study in the rat. J Comp Neurol, 2004. 468(3): p. 425-40

22. Bachen, E., Manuck, S.B., Cohen, S. Sympathetic blockade ameliorates cellular immune responses to acute psychological stress. in 9th Annual Conference on Clin. Immun. 1994

23. Cunnick, J.E., et al., Shock-induced modulation of lymphocyte responsiveness and natural killer activity: differential mechanisms of induction. Brain Behav Immun, 1988. 2(2): p. 102-13

24. Howlett, T.A. and L.H. Rees, Endogenous opioid peptides and hypothalamo-pituitary function. Annu Rev Physiol, 1986. 48: p. 527-36

25. Sluka, K.A., N.B. Lawand, and K.N. Westlund, Joint inflammation is reduced by dorsal rhizotomy and not by sympathectomy or spinal cord transection. Ann Rheum Dis, 1994. 53(5): p. 309-14

26. Leonard, B.E. and A. Myint, The psychoneuroimmunology of depression. Hum Psychopharmacol, 2009. 24(3): p. $165-75$

27. Hattori, S., et al., Enriched environments influence depression-related behavior in adult mice and the survival of newborn cells in their hippocampi. Behav Brain Res, 2007. 180(1): p. 69-76

28. Wichers, M.C., et al., IDO and interferon-alpha-induced depressive symptoms: a shift in hypothesis from tryptophan depletion to neurotoxicity. Mol Psychiatry, 2005. 10(6): p. 538-44

29. Ananth, S., Building healing spaces. Explore (NY), 2008. 4(6): p. 392-3

30. Eckersley, R., The mixed blessings of material progress: diminishing returns in the pursuit of hapipiness. Journal of Happiness Studies, 2000. 1: p. 267-292

31. Potter, H.A., Psychology and technical civilization. Journal of Applied Psychology, 1935. 19(1): p. 1-8

32. Rescher, N., Technological Progress and Human Happiness. Philosophic Exchange Brockport N.Y., 1979. 2(5): p. 65-79

33. Peseschkian, N., Positive psychotherapy: a transcultural and interdisciplinary approach to psychotherapy. Psychother Psychosom, 1990. 53(1-4): p. 39-45 
Summary 
In this thesis, it was our aim to study the effect of an enriched environment (EE) on inflammatory pain, using a rat model of knee inflammation. This investigation mainly focused on behavioral outcomes.

In chapter 1, we introduced the field of postoperative inflammatory pain in the clinic as well as in experimental pain research with a special emphasis on the impact of the environment on it.

In chapters 2 to 4 , we first addressed a methodological question about pain measurement: is the CatWalk, a gait computerized analysis technique, an appropriate tool to assess pain-induced gait changes in a rat model of carrageenan-induced inflammatory pain? In chapter 2 , we demonstrated that, in the first 48 hours following the carrageenan injection, indeed the CatWalk technique allows objective analysis of gait changes, which at the same time parallel the development of mechanical hyperalgesia as assessed by the von Frey test. In chapter 4, we confirmed the causality of pain in the observed CatWalk-based gait changes by studying the effect of Fentanyl, an opioid analgesic, on the carrageenan-induced gait changes. In chapter 3, the use of the CatWalk technique was studied in the chronic phase of the knee inflammation. Our results indicated that, as of one week after the carrageenan injection, the CatWalk parameters affected by the carrageenan knee injection do no longer differ from pre-operative values whereas at the same time the von Frey withdrawal threshold indicates a mechanical hyperalgesia which lasts up to 4 weeks post-injection. These data suggest that, as of one week after the carrageenan injection, the animals do no longer adapt their gait to pain and the phenomenon of habituation is suspected to take place. In conclusion, our data indicate that the CatWalk is a potent and objective technique to assess paininduced gait changes in correlation with mechanical allodynia in the carrageenaninduced inflammatory pain model. Nevertheless, its use is restricted to the acute phase of this model. Second, our results also indicate that a simultaneous use of both von Frey and CatWalk tests to assess pain in experimental pain research will result in a better and more reliable picture of the whole pain experience in the rat.

The following chapters focused on the influence of housing in an EE on behavioral pain recovery in the model of carrageenan-induced chronic knee inflammation. The EE consists of ten rats housed in a large cage $(\mathrm{L} \times \mathrm{W} \times \mathrm{H}=2.0 \times 1.0 \times 0.8 \mathrm{~m})$ including extra nesting materials, tunnels and running wheels, as compared with standard housing (S-) were animals are singly housed in standard cage $(\mathrm{L} \times \mathrm{W} \times \mathrm{H}=0.5 \times 0.2 \times 0.2 \mathrm{~m})$ . Hence, the EE provides both greater physical and social stimulations. In chapter 5, EE-housing following the carrageenan knee injection led to a duration of mechanical hyperalgesia of 3 weeks in the ipsilateral paw, as compared with 4 weeks when rats are S-housed. Histological studies of the rat spinal dorsal horn indicated the astrocytes to 
be involved in this environmentally-driven reduction of inflammatory pain duration. Chapter 6 aimed at studying the effect of a pre-inflammation EE-housing on inflammatory pain duration. Comparison with post-inflammation EE-housing was studied as well as comparison with the combination of both a pre- and post-inflammation EE-housing. Our data demonstrated that a pre-inflammation EE-housing leads to a similar reduction of one week of the inflammatory pain duration than a post-inflammation EE-housing does. Besides, we also demonstrated that a combination of housing in an EE prior to and after the carrageenan injection leads to a duration of the mechanical allodynia of 2 weeks, as compared with 4 weeks in S-housed rats.

In chapter 7, we investigated to what extend each aspect of the EE differentially affects the development of inflammatory pain. In this study, four housing conditions were studied: a physically enriched environment (PE), a socially enriched environment (SE), a physically and socially enriched environment (EE) and a restricted standard environment (S-housing). Our results confirmed that the duration of carrageenaninduced mechanical allodynia if rats are kept in a S-housing is of 4 weeks and is of 3 weeks if rats are housed in an EE. Similarly, housing in a physically enriched environment $(\mathrm{PE})$ also resulted in a reduction of the duration of mechanical allodynia of one week. And if housed in a socially enriched environment (SE), the carrageenaninduced mechanical allodynia lasted for 3 weeks and an half. Based on these results, we first concluded that both physical and social aspects of EE are involved in the reduction of inflammatory pain duration. In this experimental setting, the physical component has a major effect over the social aspect of the EE. Second, our data demonstrate that the beneficial effects on inflammatory pain duration of both components are inter-dependent, i.e. the effect of the social enrichment depends on the physical aspects of the environment (enriched or not) and reversely.

In chapter 8, we first discussed the use of the CatWalk technique in the field of experimental pain. Second, we discussed the behavioral outcomes from the different studies investigating the effect of an enriched environment on inflammatory pain. A general underlying biological mechanism was suggested.

In conclusion, the current work indicates a major effect of the environment on the development of carrageenan-induced inflammatory pain, prior to as well as after the induction of inflammation. Besides, our results emphasize the importance of both the physical and social enrichment of housing conditions. Human studies are needed to confirm the clinical implications of this work. Nevertheless, these findings urge the future development of a clinical healing environment including both physical and social enrichment, prior to and after the operation for post-operative inflammatory pain patients. 

Aknowledgment 
The successful outcome of this $\mathrm{PhD}$ project is certainly due to a lot of valuable support from colleagues and friends that I would like to thank here, as a final step of my work.

First of all, I'd like to thank Pr dr Marco Marcus, my promoter. Thank you for the clinical touch you brought in this work as well as for your support and guidance. The way you try to help me in deciding where I want to go to (though it was not always an easy work!) and to bring me further away in my projects was priceless.

Then of course, many thanks to Dr Bert Joosten, my co-promoter. Working in your team for four years was definitely a privilege. I'm impressed by the way you constantly listen to the others' needs and try to actively support each of us in our own way. Thank you for that and for the human and nice atmosphere you create, at every werkbespreking (at 9.00 sharp!) and in our group.

Close to them, the Experimental Pain team: Wiel Honig, Ronald Deumens, Michiel Truin, Robby Jaken, Guido Koopmans, Joris Coonen, Helwin Smits, Sabien van Neerven, Liesbeth Knaepen and Sofie Janssen. With special thanks to Wieleke, for your persistent and free Dutch lessons :-) and your permanent help during these four years! Thank you also to Michiel ("Mickael”!) for the smoking breaks as well as to Robby, my Belgian mate, and Ronald for all the nice moments we've had in Maastricht and in travels! Thank you also to the students that worked on my project: Ellen Konings, Nicolle Helgers and Emiel van der Vorst.

Then, I'd like to thank the Dolphys team, from Eindhoven, with whom I worked for 4 years. Many thanks to Dirk van Asseldonck as well as Naomi, Fabienne and Leon for the very nice and interesting collaboration. I wish you all the best for your future projects and developments.

I also would like to thank Pr dr M. van Kleef as well as Dr J. Patijn for their support in my work and the inspiring scientific discussions we've had. Thank you also to Pr $\mathrm{dr}$ H. Steinbush since this work mainly took place within the Institute of Mental Health and Neuroscience. 
Of course, many thanks to Pr GC Carli and Pr F Farabollini and the team of the department of Human Physiology in Siena (Itlay): Giulia, Ricardo and Daniele. Thank you for this collaboration! This "toscanian" project was great and I've learned a lot from you. Thank you to Enrico and Lydia, my Brazilian and American friends from il Collegio Santa Chiara e il Refugio who also contributed to make my Italian stay an unforgettable time! All together, I'm very proud and happy of this sunny and Italian touch in my work, so thank you all again.

Concerning the administrative part of this work, I'd like to thank Hermina Gielen from Anesthesiology for her active support especially by the end of the thesis. And thank you to Michal Sławiński for the type-setting and printing of the book. This polish collaboration was as easy as nice.

Last but not least, I'd like to thank G, Soi, Mattch, Ritch, Thierry, Méla, Florence and Cath, éternels compagnons d'humour et de travail $\odot$.) And thanks to all the others that have supported me during the last four years and contributed to brighten my days and my work (e.g. Luca, Abraham, Mu, Matteo, Jodi, Chiara from Maastricht and all my family and friends from LLN or from scouting!). And finally, thank you from my heart to my parents and brothers that support me in all my projects. Even though these projects may sometimes be crazy or unreasonable, you are always there for me and it definitely means the world to me.

"Quand on est arrivé au but de son voyage, on dit que la route a été bonne" (Proverbe chinois)... Merci à vous tous d'avoir partagé celle-ci avec moi et puissent nos chemins souvent encore se croiser!

"Once you have reached your goal, you say that the journey was good" (Chinese proverb).... Thank you all for sharing this journey with me. May our paths often cross again! 

Curriculum vitae 

Anne Gabriel was born on the 6th of August 1977 in Rocourt (Belgium) and grew up in Siegen (Germany), in Brussels (Belgium) and finally in Liège (Belgium). In 1995, she graduated from secondary school at Lycée Saint-Benoit Saint-Servais in Liège. In 1999, she received her Master's degree in Chemistry (with specialization in Biochemistry) at the Université Catholique de Louvain (UCL) in Louvain-la-Neuve (Belgium). In 2000, she received her Teaching Diploma from the Facultés Notre-Dame de la Paix (FUNDP) in Namur (Belgium). She worked on research projects in biochemistry until 2003, including a project as a guest researcher at the Institut Polytechnique de Lorraine in Nancy (France). She then worked for one year as a project manager in an NPO, in charge of the training coordination and of the recruitment of volunteers. Then, back to sciences, she started her $\mathrm{PhD}$ project at the Pain Management and Research Center from the Academic Hospital in Maastricht (The Netherlands), including a project as a guest researcher at the Universita degli studi di Siena in Siena (Italy). Beside her professional enrollment, she has been volunteering as a trainer and a team manager in a youth organization where she is now in charge of international projects. In order to complete her scientific background, she was recently educated in ICH-GCP and clinical research coordination at the Clinical College in Wemmel (Belgium). 



\section{List of Publications}




\section{Journal articles}

- Gabriel AF, Honig WM, Marcus MA, Joosten EA. Environmental housing affects the duration of mechanical allodynia and the spinal astroglial activation in a rat model of chronic inflammatory pain. Brain Research 2009 Jun 18; 1276: 83-90

- Gabriel AF, Marcus MA, Walenkamp GH, Joosten EA. The CatWalk method: assessment of mechanical allodynia in experimental chronic pain? Behavioral Brain Research 2009 Mar 17; 198 (2); 477-80

- Gabriel AF, Marcus MA, Honig WM, Walenkamp GH, Joosten EA. The CatWalk method: A detailed analysis of behavioral changes after acute inflammatory pain in the rat. J Neurosci Methods. 2007 Jun 15; 163 (1): 9-16

- Berger V, Gabriel AF, Sergent T, Trouet A, Larondelle Y, Schneider YJ. Interaction of ochratoxin A with human intestinal Caco-2 cells: possible implication of a multidrug resistance-associated protein (MRP2). Toxicol Lett. 2003 Apr 11;140$141: 465-76$

\section{Publications in progress}

- Gabriel AF, Paoletti G, Marcus MA, Joosten EA, Carli GC. Enriched environment and the recovery from inflammatory pain: social versus physical aspects and their interaction. Pain 2009 - Submitted

- Gabriel AF, Honig WM, Marcus MA, Joosten EA. Preoperative housing in an enriched environment significantly reduces the duration of post-operative pain in a rat model of knee inflammation - Submitted

- M Sommer, AF Gabriel, J Patijn, M van Kleef, B Joosten, HF Gramke, MAE Marcus. Acute Pain Treatment: do we need a shift in direction? - Review submitted.

- JTF Keurentjes, MF Kemmere, H Bruinewoud, MA Vertommen, SA Rovers, R Hoogenboom, LF Stemkens, FL Péters, NJ Tielen, DT van Asseldonk, AF Gabriel, MA Marcus.. Externally Triggered Glass Transition Switch for Localised On-Demand Drug Delivery. PNAS 2009 - Submitted

- Gabriel AF, Honig WM, Marcus MA, Joosten EA. The CatWalk method: a sensitive and objective tool for preclinical assessment of analgesic drugs - In preparation. 


\section{Abstracts - Oral presentations}

1. A.F. Gabriel, W.M.M. Honig, M.A.E. Marcus, E.A.J. Joosten (2008). 6th International Conference on Methods and Techniques in Behavioral Research, Maastricht (The Netherlands). Measuring Inflammatory Pain: the CatWalk method.

2. A.F. Gabriel, M.A.E. Marcus, W.M.M. Honig, R. Deumens, M. van Kleef, E.A.J. Joosten (2007). 4th Science's Day, Dutch Anesthesiologists society (NVA), Amsterdam (The Netherlands). Recovery from inflammatory pain in the rat: influence of the socio-physical environment.

3. A.F. Gabriel, W.M.M. Honig, M.A.E. Marcus, E.A.J. Joosten (2007). 11th Euron PhD Student Day (European graduate school of neuroscience), Bruxelles (Belgium). CatWalk parameters as a measure of a carrageenan-induced inflammatory pain in the acute but not chronic phase.

4. A.F. Gabriel, W.M.M. Honig, M. Marcus, E.A.J. Joosten (2006). 10th Annual meeting of Dutch Anesthesiologists society (NVA), Maastricht (The Netherlands). Acute pain research: use of the Catwalk for detailed analysis of behavioral changes.

5. A.F. Gabriel, W.M.M. Honig, M. Marcus, M. van Kleef, E.A.J. Joosten (2006). $3 d$ Science's Day, Dutch Anesthesiologists society (NVA) (The Netherlands). Objective gait analysis as a measure of acute inflammatory pain in the rat.

\section{Abstracts - Poster presentations}

1. A.F. Gabriel, W.M.M. Honig, R. Deumens, M.A.E. Marcus, E.A.J. Joosten (2008). Annual Society for Neuroscience Meeting, Washington DC (USA). Catwalk-based pain induced gait adaptation: sensitive and objective preclinical assessment of analgesic drugs.

2. A.F. Gabriel, W.M.M. Honig, R. Deumens, M.A.E. Marcus, E.A.J. Joosten (2008). NeuPSIG - Neuropathic Pain Satellite congress to the Glasgow 2008 World congress on Pain, London (UK). The CatWalk method: a sensitive and objective tool for preclinical assessment of analgesic drug.

3. A.F. Gabriel, Marcus, E.A.J, W.M.M. Honig, R. Deumens, M.A.E. Joosten (2007). Annual Society for Neuroscience Meeting, San Diego (USA). Housing in an enriched environment enhances recovery from carrageenan-induced inflammatory pain. 
4. R.J.P. Jaken, A.F. Gabriel, G.C.Koopmans, M. van Kleef, M.A. Marcus, E.A.J. Joosten, R. Deumens (2007). Annual Society for Neuroscience Meeting, San Diego (USA). Innovative use of the CatWalk for detection of gait changes after rat spinal cord injury and inflammatory pain.

5. A.F. Gabriel, W.M.M. Honig, R. Deumens, M.A.E. Marcus, E.A.J. Joosten (2007). $2 d$ International Congress on Neuropathic pain, Berlin (Germany). CatWalk analysis and pain research: gait parameters as a measure of inflammatory pain in the acute but no chronic phase.

6. R.J.P. Jaken, A.F. Gabriel, E.A.J. Joosten, G.C. Koopmans, R. Deumens (2007). $2 d$ International Congress on Neuropathic Pain, Berlin (Germany). Assessment of neuropathic pain and locomotor deficits using the CatWalk gait analysis.

7. A.F. Gabriel, W.M.M. Honig, M.A.E. Marcus, E.A.J. Joosten (2007). 6th Institute's Day Brain and Behavior, Maastricht (The Netherlands). CatWalk analysis and pain research: gait parameters as a measure of inflammatory pain in the acute but no chronic phase.

8. R.J.P. Jaken, A.F. Gabriel, E.A.J. Joosten, G.C. Koopmans, R. Deumens (2006). 6th International Symposium on Experimental Spinal Cord Repair and Regeneration, Brescia (Italy). The CatWalk gait analysis as a valuable tool to assess both interlimb coordination and pain-induced gait adaptations in rodents.

9. A.F. Gabriel, W.M.M. Honig, M.A.E. Marcus, E.A.J. Joosten (2006). 5th congress of the European federation of IASP chapters (EFIC), Istanbul (Turkey). Detailed analysis of behavioral changes after acute pain in the rat: the CatWalk method.

10. A.F. Gabriel, W.M.M. Honig, M.A.E. Marcus, E.A.J. Joosten (2006). 10th EndoNeuro meeting, Doorwerth (The Netherlands). Acute pain research: use of the Catwalk for detailed analysis of behavioral changes.

11. A.F. Gabriel, W.M.M. Honig, M.A.E. Marcus, E.A.J. Joosten (2006). 10th Euron PhD Student Day (European graduate school of neuroscience), Maastricht (The Netherlands). Use of the CatWalk for behavioral analysis of acute inflammatory pain. 